\title{
Molybdenum and Tungsten Alkylidene Complexes That Contain a 2-Pyridyl-substituted Phenoxide Ligand
}

\author{
Peter E. Sues, Jeremy M. John, Konstantin V. Bukhryakov, Richard R. Schrock, and Peter Müller \\ Department of Chemistry, Massachusetts Institute of Technology, Cambridge, Massachusetts 02139 \\ email rrs@mit.edu
}

\section{Table of Contents}

Syntheses of known compounds

S2

Spectroscopic Data

S3-S17

Catalytic Data

$\mathrm{S} 18-\mathrm{S} 20$

X-Ray Diffraction Data

S21-S41

GPC, IR, and Proton and Carbon NMR data for polyA, polyB and polyC

S42-S45

Observed consumption monomer in ROMP reactions

S45

References

S46 
SYNTHESES of known compounds

Synthesis of 2,6-Dibromo-4-methylphenol. The product was synthesized via a modified procedure reported by Garcia et al. PCT Int. Appl., 2007128492 A1. ${ }^{1}$ A solution of $71.7 \mathrm{~g}$ of bromine (3.5 eq., 448.9 $\mathrm{mmol}$ ) in $320 \mathrm{~mL}$ glacial acetic acid was added dropwise to a solution of $23.6 \mathrm{~g} p$-cresol (1 eq., 128.3 $\mathrm{mmol}$ ) in $48 \mathrm{~mL}$ acetic acid and $132 \mathrm{~mL}$ water over the course of $2.5 \mathrm{~h}$ in a water bath. After addition was complete, the solution was stirred for an additional $0.5 \mathrm{~h}$ at room temperature and then poured slowly into $800 \mathrm{~mL}$ water. A precipitate formed and was collected by suction filtration. The solid was dissolved in $200 \mathrm{~mL}$ of $\mathrm{CH}_{2} \mathrm{Cl}_{2}$ and the mixture was transferred to a separatory funnel. The product was sequentially washed with $200 \mathrm{~mL}$ of saturated $\mathrm{Na}_{2} \mathrm{CO}_{3}, \mathrm{Na}_{2} \mathrm{~S}_{2} \mathrm{O}_{3}$ and brine. The organic layer was collected over anhydrous $\mathrm{MgSO}_{4}$, stirred for $30 \mathrm{~min}$, filtered and the solvent removed using a rotary evaporator. The white solid that was obtained was then dried using a high vacuum line prior to use; yield $46.2 \mathrm{~g}$ (80\%). Spectroscopic data were consistent with those reported by Garcia et al.

Synthesis of 2,6-Dibromo-4-methylanisole. Neat Mel (2 equiv, $49.3 \mathrm{~g}, 347.2 \mathrm{mmol}$ ) was added to a suspension of 46.2g 2,6-dibromo-4-methylphenol (1 eq., $173.6 \mathrm{mmol}$ ) and excess anhydrous $\mathrm{K}_{2} \mathrm{CO}_{3}$ in $100 \mathrm{~mL}$ acetone. The mixture was heated at $65^{\circ} \mathrm{C}$ overnight and the flask was allowed to cool to room temperature (reaction completion was confirmed by thin layer chromatography; product Rf approx. 0.9 using 1:4 ethyl acetate:hexane). The unreacted $\mathrm{K}_{2} \mathrm{CO}_{3}$ was filtered off and the solvent was removed using a rotary evaporator to yield 2,6-dibromo-4-methylanisole as viscous yellow oil. The oil was distilled under high vacuum ( $85{ }^{\circ} \mathrm{C}$ at $500 \mathrm{mmHg}$ ) prior to use; yield $46.0 \mathrm{~g}$ (95\%). Spectroscopic data were consistent with those reported in the literature. ${ }^{2}$

Synthesis of 3-Bromo-2-methoxy-2',4',5,6'-tetramethyl-1,1'-biphenyl. The product was synthesized via a modified procedure reported by Koch et al. ${ }^{3}$ A solution of $100 \mathrm{~mL} 1.0 \mathrm{M} \mathrm{MesMgBr}$ in THF (1 eq., $100 \mathrm{mmol}$ ) was carefully added to a solution of $28.0 \mathrm{~g}$ 2,6-dibromo-4-methylanisole (1 eq., $100 \mathrm{mmol}$ ) and $2.31 \mathrm{~g} \mathrm{Pd}\left(\mathrm{PPh}_{3}\right)_{4}(0.02$ eq., $2 \mathrm{mmol})$ in $100 \mathrm{~mL}$ toluene. The reaction was heated at 100 ${ }^{\circ} \mathrm{C}$ for $72 \mathrm{~h}$. The reaction was allowed to cool to room temperature before transferring the mixture to a $500 \mathrm{~mL}$ Erlenmeyer flask. The solution was chilled to $0{ }^{\circ} \mathrm{C}$ with an ice bath and $200 \mathrm{~mL}$ of $2 \mathrm{~N} \mathrm{HCl}$ were added in portions. The organic layer was then separated and the aqueous layer washed twice with 100 $\mathrm{mL} \mathrm{CH} \mathrm{Cl}_{2}$. The combined organic layers were dried over anhydrous $\mathrm{MgSO}_{4}$. The mixture was filtered before and the solvent was removed from the filtrate using a rotary evaporator. The resulting oil was purified using flash chromatography (using hexane as the eluent) followed by recrystallization from methanol at $-30{ }^{\circ} \mathrm{C}$; yield $14.4 \mathrm{~g}(45 \%)$. HRMS for $\mathrm{C}_{17} \mathrm{H}_{19} \mathrm{BrO}$ : Theoretical [M+H]: 319.0692. Found [M+H]: 319.0695 .

Synthesis of 2,6-Diiodo-4-methylphenol. The product was synthesized via a modified procedure reported by Omura. ${ }^{4}$ At room temperature, $20.0 \mathrm{~g} p$-cresol ( 1 eq., $0.185 \mathrm{~mol}$ ) was added to a solution of $31.1 \mathrm{~g} \mathrm{KOH}$ ( 3 eq., $0.555 \mathrm{~mol}$ ) in $300 \mathrm{~mL} \mathrm{MeOH.} \mathrm{Over} \mathrm{a} 5 \mathrm{~min}$ period, $91.5 \mathrm{~g}$ iodine (2 eq., $0.361 \mathrm{mmol}$ ) was added portionwise and then the reaction was refluxed for 10 minutes. A precipitate formed and the suspension was cooled to room temperature. The reaction was quenched with $15.2 \mathrm{ml}$ of $35 \%$ wt. aq. $\mathrm{HCl}$ (1 eq., $0.185 \mathrm{~mol}$ ), and the precipitate was removed through filtration. The solvent of the filtrate was then removed via distillation leaving a dark residue. The product was extracted in 
refluxing $n$-hexane, and decanted from the remaining insoluble residue. Cooling the $n$-hexane solution to $-78{ }^{\circ} \mathrm{C}$ afforded a yellow precipitate, which was isolated by filtration and dried under vacuum; yield $42.9 \mathrm{~g}(65 \%)$. Spectroscopic data were consistent with those reported by Omura.

\section{SPECTROSCOPIC DATA}

3-bromo-2-methoxy-2',4',5,6'-tetramethyl-1,1'-biphenyl. ${ }^{1} \mathrm{H} \mathrm{NMR}\left(500 \mathrm{MHz}, \mathrm{CDCl}_{3}\right): \delta 7.37(\mathrm{~m}, 1 \mathrm{H}$, Aromatic-H), $6.93(\mathrm{~s}, 2 \mathrm{H}$, Aromatic- $\mathrm{H}), 6.80(\mathrm{~m}, 1 \mathrm{H}$, Aromatic- $\mathrm{H}), 3.41\left(\mathrm{~s}, 3 \mathrm{H}, \mathrm{OCH}_{3}\right), 2.33\left(\mathrm{~s}, 3 \mathrm{H}, \mathrm{CH}_{3}\right)$, $2.31\left(\mathrm{~s}, 3 \mathrm{H}, \mathrm{CH}_{3}\right)$, and $2.04\left(\mathrm{~s}, 6 \mathrm{H}, \mathrm{CH}_{3}\right)$ ppm. ${ }^{13} \mathrm{C} \mathrm{NMR}\left(125 \mathrm{MHz}, \mathrm{CDCl}_{3}\right): \delta 152.2$ (s, Aromatic-C), 137.1 (s, Aromatic-C), 136.2 (s, Aromatic-C), 135.4 (s, Aromatic-C), 135.0 (s, Aromatic-C), 134.6 (s, Aromatic-C), 132.7 (s, Aromatic-CH), 131.4 (s, Aromatic- $\mathrm{CH}$ ), 128.1 (s, Aromatic-CH), 117.3 (s, Aromatic-C), 60.2 (s, $\left.\mathrm{OCH}_{3}\right), 21.2\left(\mathrm{~s}, \mathrm{CH}_{3}\right), 20.7\left(\mathrm{~s}, \mathrm{CH}_{3}\right)$, and $20.6\left(\mathrm{~s}, \mathrm{CH}_{3}\right) \mathrm{ppm}$.

2-(2-methoxy-2',4',5,6'-tetramethyl-[1,1'-biphenyl]-3-yl)pyridinium chloride. ${ }^{1} \mathrm{H} \mathrm{NMR}\left(500 \mathrm{MHz}, \mathrm{CDCl}_{3}\right.$ : $\delta 17.52$ (bs, $1 \mathrm{H}, \mathrm{NHCl}$ ), 9.06 (d, $1 \mathrm{H}$, Aromatic- $\mathrm{H}, J=5.0 \mathrm{~Hz}$ ), 8.33 (t, $1 \mathrm{H}$, Aromatic- $\mathrm{H}, J=10 \mathrm{~Hz}$ ), 8.28 (d, $1 \mathrm{H}$, Aromatic- $\mathrm{H}, J=10 \mathrm{~Hz}), 7.84(\mathrm{~s}, 1 \mathrm{H}$, Aromatic-H), $7.80(\mathrm{t}, 1 \mathrm{H}$, Aromatic- $\mathrm{H}, J=5.0 \mathrm{~Hz}), 7.10(\mathrm{~d}, 1 \mathrm{H}$, Aromatic- $\mathrm{H}, \mathrm{J}=5.0 \mathrm{~Hz}), 6.95\left(\mathrm{~s}, 2 \mathrm{H}\right.$, Aromatic-H), $3.22\left(\mathrm{~s}, 3 \mathrm{H}, \mathrm{OCH}_{3}\right), 2.45\left(\mathrm{~s}, 3 \mathrm{H}, \mathrm{CH}_{3}\right), 2.33\left(\mathrm{~s}, 3 \mathrm{H}, \mathrm{CH}_{3}\right)$, and 2.09 (s, 6H, CH 3 ) ppm. ${ }^{13} \mathrm{C} \mathrm{NMR} \mathrm{(125} \mathrm{MHz,} \mathrm{CDCl} 3$ ): $\delta 153.62$ (s, Aromatic-C), 151.59 (s, Aromatic-C), 144.39 (s, Aromatic-CH), 142.16 (s, Aromatic-CH), 137.55 (s, Aromatic-C), 137.38 (s, Aromatic- $\mathrm{CH}$ ), 136.33 (s, Aromatic-C), 135.02 (s, Aromatic-C), 134.38 (s, Aromatic-C), 134.13 (s, Aromatic-C), 131.08 (s, Aromatic-CH), 128.58 (s, Aromatic-CH), 128.47 (s, Aromatic- $\mathrm{CH}), 124.07$ (s, Aromatic- $\mathrm{CH}$ ), 123.4 (s, Aromatic-C), $60.91\left(\mathrm{~s}, \mathrm{OCH}_{3}\right), 21.21\left(\mathrm{~s}, \mathrm{CH}_{3}\right), 20.91\left(\mathrm{~s}, \mathrm{CH}_{3}\right)$, and $20.87\left(\mathrm{~s}, \mathrm{CH}_{3}\right) \mathrm{ppm}$.

2',4',5,6'-tetramethyl-3-(pyridin-2-yl)-[1,1'-biphenyl]-2-ol. ${ }^{1} \mathrm{H} N M R\left(500 \mathrm{MHz}, \mathrm{C}_{6} \mathrm{D}_{6}\right): \delta 14.45(\mathrm{~s}, 1 \mathrm{H}, \mathrm{OH})$, $7.73(\mathrm{~d}, 1 \mathrm{H}$, Aromatic- $\mathrm{CH}, \mathrm{J}=5.0 \mathrm{~Hz}), 7.41(\mathrm{~d}, 1 \mathrm{H}$, Aromatic- $\mathrm{CH}, J=1.6 \mathrm{~Hz}), 7.32(\mathrm{~d}, 1 \mathrm{H}$, Aromatic-CH, $J=$ $8.3 \mathrm{~Hz}), 7.01-6.94(\mathrm{~m}, 4 \mathrm{H}$, Aromatic- $\mathrm{CH}), 6.39-6.34(\mathrm{~m}, 1 \mathrm{H}$, Aromatic- $\mathrm{CH}), 2.33\left(\mathrm{~s}, 6 \mathrm{H}, \mathrm{CH}_{3}\right), 2.26(\mathrm{~s}, 3 \mathrm{H}$, $\mathrm{CH}_{3}$ ), and $2.22\left(\mathrm{~s}, 3 \mathrm{H}, \mathrm{CH}_{3}\right)$ ppm. ${ }^{13} \mathrm{C}$ NMR $\left(125 \mathrm{MHz}, \mathrm{C}_{6} \mathrm{D}_{6}\right): \delta 159.09$ (s, Aromatic-C), 156.58 (s, AromaticC), 146.26 (s, Aromatic-CH), 137.55 (s, Aromatic- $\mathrm{CH}$ ), 137.10 (s, Aromatic-C), 136.6 (s, Aromatic-CH), 134.57 (s, Aromatic-CH), 131.62 (s, Aromatic-C), 128.77 (s, Aromatic-CH), 127.54 (s, Aromatic-C), 126.19 (s, Aromatic-CH), 121.20 (s, Aromatic-CH), 119.52 (s, Aromatic- $\mathrm{CH}$ ), 119.32 (s, Aromatic-C), 21.58 (s, $\left.\mathrm{CH}_{3}\right)$, and $21.22\left(\mathrm{~s}, \mathrm{CH}_{3}\right) \mathrm{ppm}$.

3-iodo-2',4',6'-triisopropyl-5-methyl-[1,1'-biphenyl]-2-ol or $\mathrm{H}[\mathrm{MesON}] .{ }^{1} \mathrm{H} \mathrm{NMR}\left(400 \mathrm{MHz}, \mathrm{CDCl}_{3}\right): \delta$ 7.55 (s, 1H, Aromatic-H), 7.10 (s, 2H, Aromatic-H), 6.83 (s, 1H, Aromatic-H), 4.97 (s, 1H, OH), 2.94 (sept, $1 \mathrm{H}, \mathrm{CHCH}_{3}, J=6.9 \mathrm{~Hz}$ ), 2.55 (sept, $2 \mathrm{H}, \mathrm{CHCH}_{3}, J=6.9 \mathrm{~Hz}$ ), $2.27\left(\mathrm{~s}, 3 \mathrm{H}, \mathrm{CH}_{3}\right), 1.30$ (d, $\left.6 \mathrm{H}, \mathrm{CHCH}_{3}, J=6.9 \mathrm{~Hz}\right)$, $1.04\left(\mathrm{~d}, 6 \mathrm{H}, \mathrm{CHCH}_{3}, J=6.9 \mathrm{~Hz}\right)$, and $0.99\left(\mathrm{~d}, 6 \mathrm{H}, \mathrm{CHCH}_{3}, J=6.9 \mathrm{~Hz}\right) \mathrm{ppm} .{ }^{13} \mathrm{C} \mathrm{NMR}\left(101 \mathrm{MHz}, \mathrm{CDCl}_{3}\right): \delta$ 150.5 (s, Aromatic-C), 149.7 (s, Aromatic-C), 148.0 (s, Aromatic-CH), 138.5 (Aromatic-CH), 131.9 (s, Aromatic-C), 131.5 (s, Aromatic-C), 129.9 (s, Aromatic-C), 126.2 (s, Aromatic-C), 121.6 (s, Aromatic- $\mathrm{CH}$ ), 83.0 (s, Aromatic- $\mathrm{Cl}), 34.5\left(\mathrm{~s}, \mathrm{CHCH}_{3}\right), 30.6\left(\mathrm{~s}, \mathrm{CHCH}_{3}\right), 24.7\left(\mathrm{~s}, \mathrm{CH}_{3}\right), 24.16\left(\mathrm{~s}, \mathrm{CH}_{3}\right), 24.04\left(\mathrm{~s}, \mathrm{CH}_{3}\right)$, and $20.2\left(\mathrm{~s}, \mathrm{CH}_{3}\right) \mathrm{ppm}$.

2',4',6'-triisopropyl-5-methyl-3-(pyridin-2-yl)-[1,1'-biphenyl]-2-ol or $\mathrm{H}\left[T\right.$ TripON]. ${ }^{1} \mathrm{H}$ NMR (400 $\mathrm{MHz}$, $\left.\mathrm{CDCl}_{3}\right): \delta 14.15(\mathrm{~s}, 1 \mathrm{H}, \mathrm{OH}), 8.44(\mathrm{dd}, 1 \mathrm{H}$, Aromatic- $\mathrm{CH}, J=5.0,0.8 \mathrm{~Hz}), 8.00(\mathrm{~d}, 1 \mathrm{H}$, Aromatic- $\mathrm{CH}, J=8.4$ 
$\mathrm{Hz})$, 7.86-7.82 (m, 1H, Aromatic-CH), $7.64(\mathrm{~d}, 1 \mathrm{H}$, Aromatic- $\mathrm{CH}, J=1.9 \mathrm{~Hz}), 7.22(\mathrm{ddd}, 1 \mathrm{H}$, Aromatic- $\mathrm{CH}, J$ $=7.4,5.1,0.9 \mathrm{~Hz}), 7.09(\mathrm{~s}, 2 \mathrm{H}$, Aromatic- $\mathrm{CH}), 6.98(\mathrm{~d}, 1 \mathrm{H}$, Aromatic- $\mathrm{CH}, J=1.9 \mathrm{~Hz}), 2.95\left(\right.$ sept $, 1 \mathrm{H}, \mathrm{CHCH}_{3}$, $J=6.9 \mathrm{~Hz}$ ), 2.71 (sept, $2 \mathrm{H}, \mathrm{CHCH}_{3}, J=6.9 \mathrm{~Hz}$ ), $2.37\left(\mathrm{~s}, 3 \mathrm{H}, \mathrm{CH}_{3}\right), 1.32\left(\mathrm{~d}, 6 \mathrm{H}, \mathrm{CHCH}_{3}, J=6.9 \mathrm{~Hz}\right), 1.15(\mathrm{~d}$, $6 \mathrm{H}, \mathrm{CHCH}_{3}, J=6.9 \mathrm{~Hz}$ ), and $1.10\left(\mathrm{~d}, 6 \mathrm{H}, \mathrm{CHCH}_{3}, J=6.9 \mathrm{~Hz}\right) \mathrm{ppm} .{ }^{13} \mathrm{C} \mathrm{NMR}\left(101 \mathrm{MHz}, \mathrm{CDCl}_{3}\right): \delta 158.3(\mathrm{~s}$, Aromatic-C), 155.7 (s, Aromatic-C), 147.7 (s, Aromatic-CH), 146.9 (s, Aromatic-CH), 145.9 (s, Aromatic$\mathrm{CH}), 137.9$ (s, Aromatic-CH), 134.4 (s, Aromatic-CH), 133.2 (s, Aromatic-C), 130.0 (s, Aromatic-C), 126.9 (s, Aromatic-C), 125.5 (s, Aromatic-C), 121.4 (s, Aromatic-CH), 120.8 (s, Aromatic-CH), 119.5 (s, AromaticC), 118.1 (s, Aromatic-C), $34.4\left(\mathrm{~s}, \mathrm{CHCH}_{3}\right), 30.8\left(\mathrm{~s}, \mathrm{CHCH}_{3}\right), 24.7\left(\mathrm{~s}, \mathrm{CH}_{3}\right), 24.27\left(\mathrm{~s}, \mathrm{CH}_{3}\right), 24.07\left(\mathrm{~s}, \mathrm{CH}_{3}\right)$, and $21.0\left(\mathrm{~s}, \mathrm{CH}_{3}\right) \mathrm{ppm}$.

$\mathrm{H}_{2}$ [MesON]Cl. ${ }^{1} \mathrm{H}$ NMR $\left(500 \mathrm{MHz}, \mathrm{CD}_{2} \mathrm{Cl}_{2}\right.$ ) $\delta: 11.88$ (br s, $2 \mathrm{H}, \mathrm{OH}$ and $\mathrm{NH}$ ), 8.80 (dd, $1 \mathrm{H}$, Aromatic- $\mathrm{CH}, J=$ 5.8, $1.7 \mathrm{~Hz}$ ), $8.33(\mathrm{dt}, 1 \mathrm{H}$, Aromatic- $\mathrm{CH}, J=7.9,1.7 \mathrm{~Hz}$ ), $8.19(\mathrm{~d}, 1 \mathrm{H}$, Aromatic- $\mathrm{CH}, J=8.2 \mathrm{~Hz}$ ), 7.75-7.71 $(\mathrm{m}, 1 \mathrm{H}$, Aromatic- $\mathrm{CH}), 7.67-7.65(\mathrm{~m}, 1 \mathrm{H}$, Aromatic- $\mathrm{CH}), 7.12-7.09(\mathrm{~s}, 1 \mathrm{H}$, Aromatic- $\mathrm{CH}), 6.99(\mathrm{~s}, 2 \mathrm{H}$, Aromatic-CH), $2.42\left(\mathrm{~s}, 3 \mathrm{H}, \mathrm{CH}_{3}\right), 2.33\left(\mathrm{~s}, 3 \mathrm{H}, \mathrm{CH}_{3}\right)$, and $2.05\left(\mathrm{~s}, 6 \mathrm{H}, \mathrm{CH}_{3}\right)$ ppm. ${ }^{13} \mathrm{C} \mathrm{NMR}\left(125 \mathrm{MHz}, \mathrm{CD}_{2} \mathrm{Cl}_{2}\right)$

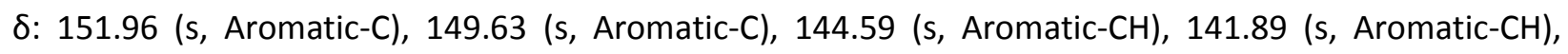
138.46 (s, Aromatic-C), 137.66 (s, Aromatic-C), 135.66 (s, Aromatic-CH), 132.10 (s, Aromatic-C), 131.57 (s, Aromatic-C), 130.33 (s, Aromatic-C), 128.92 (s, Aromatic-CH), 127.32 (s, Aromatic-CH), 124.14 (s, Aromatic- $\mathrm{CH}$ ), 119.84 (s, Aromatic-C), $21.20\left(\mathrm{~s}, \mathrm{CH}_{3}\right), 20.70\left(\mathrm{~s}, \mathrm{CH}_{3}\right)$, and $20.56\left(\mathrm{~s}, \mathrm{CH}_{3}\right) \mathrm{ppm}$.

$\mathrm{H}_{2}$ [TripON]Cl. ${ }^{1} \mathrm{H}$ NMR $\left(400 \mathrm{MHz}, \mathrm{C}_{6} \mathrm{D}_{6}\right) \delta: 8.14(\mathrm{br} \mathrm{s}, 2 \mathrm{H}, \mathrm{OH}$ and NH), $8.00(\mathrm{~d}, 1 \mathrm{H}$, Aromatic- $\mathrm{CH}, J=5.0$ $\mathrm{Hz})$, 7.32-7.28 (m, 3H, Aromatic-CH), $7.12(\mathrm{~d}, 1 \mathrm{H}$, Aromatic- $\mathrm{CH}, J=8.2 \mathrm{~Hz}), 7.09(\mathrm{~d}, 1 \mathrm{H}$, Aromatic- $\mathrm{CH}, J=$ $1.7 \mathrm{~Hz}), 6.87-6.80(\mathrm{~m}, 1 \mathrm{H}$, Aromatic- $\mathrm{CH}), 6.37-6.30(\mathrm{~m}, 1 \mathrm{H}$, Aromatic- $\mathrm{CH}), 3.01$ (sept, $2 \mathrm{H}, \mathrm{CH}\left(\mathrm{CH}_{3}\right)_{2}, J=6.8$ $\mathrm{Hz}$ ), 2.90 (sept, $\left.1 \mathrm{H}, \mathrm{CH}\left(\mathrm{CH}_{3}\right)_{2}, J=6.9 \mathrm{~Hz}\right), 2.21\left(\mathrm{~s}, 3 \mathrm{H}, \mathrm{CH}_{3}\right), 1.41\left(\mathrm{~d}, 6 \mathrm{H}, \mathrm{CH}\left(\mathrm{CH}_{3}\right)_{2}, J=6.8 \mathrm{~Hz}\right), 1.30(\mathrm{~d}, 6 \mathrm{H}$, $\left.\mathrm{CH}\left(\mathrm{CH}_{3}\right)_{2}, J=6.9 \mathrm{~Hz}\right)$, and $1.23\left(\mathrm{~d}, 6 \mathrm{H}, \mathrm{CH}\left(\mathrm{CH}_{3}\right)_{2}, J=6.8 \mathrm{~Hz}\right) \mathrm{ppm} .{ }^{13} \mathrm{C} \mathrm{NMR}\left(125 \mathrm{MHz}, \mathrm{C}_{6} \mathrm{D}_{6}\right) \delta: 157.73(\mathrm{~s}$, Aromatic-C), 155.34 (s, Aromatic-C), 148.48 (s, Aromatic-C), 147.68 (s, Aromatic-C), 144.78 (s, Aromatic$\mathrm{CH}), 138.75$ (s, Aromatic-CH), 135.17 (s, Aromatic-CH), 133.79 (s, Aromatic-C), 131.68 (s, Aromatic-C), 127.09 (s, Aromatic-CH), 121.50 (s, Aromatic-CH), 121.08 (s, Aromatic-CH), 120.98 (s, Aromatic- $\mathrm{CH}$ ), 119.63 (s, Aromatic-C), $34.94\left(\mathrm{~s}, \mathrm{CHCH}_{3}\right), 31.34\left(\mathrm{~s}, \mathrm{CHCH}_{3}\right), 24.88\left(\mathrm{~s}, \mathrm{CH}_{3}\right), 24.57\left(\mathrm{~s}, \mathrm{CH}_{3}\right), 24.49\left(\mathrm{~s}, \mathrm{CH}_{3}\right)$, and $20.85\left(\mathrm{~s}, \mathrm{CH}_{3}\right) \mathrm{ppm}$.

Li·TripON. ${ }^{1} \mathrm{H}$ NMR $\left(400 \mathrm{MHz}, \mathrm{CD}_{2} \mathrm{Cl}_{2}\right) \delta: 7.68$ (ddd, $\left.J=8.2,7.3,1.9 \mathrm{~Hz}, 1 \mathrm{H}\right), 7.60(\mathrm{dd}, J=7.4,0.9 \mathrm{~Hz}, 1 \mathrm{H})$, 7.20-7.20 (m, 1H), 7.09-7.06 (m, 3H), 6.91 (ddd, J = 7.3, 5.1, 1.3 Hz, 1H), $6.67(\mathrm{dd}, J=2.5,0.6 \mathrm{~Hz}, 1 \mathrm{H})$, 2.87-2.73 (m, 3H), $2.22(\mathrm{~s}, 3 \mathrm{H}), 1.19(\mathrm{~d}, J=6.9 \mathrm{~Hz}, 6 \mathrm{H}), 1.01$ (dd, $J=12.4,6.9 \mathrm{~Hz}, 12 \mathrm{H}) .{ }^{13} \mathrm{C}$ NMR $(125$ $\mathrm{MHz}, \mathrm{C}_{6} \mathrm{D}_{6}$ ) $\delta: 161.94$ (s, Aromatic-C), 1161.17 (s, Aromatic-C), 148.14 (s, Aromatic-C), 147.66 (s, Aromatic-C), 147.58 (s, Aromatic-CH), 138.04 (s, Aromatic-CH), 137.36 (s, Aromatic-CH), 134.27 (s, Aromatic-C), 131.54 (s, Aromatic-C), 130.95 (s, Aromatic- $\mathrm{CH}$ ), 124.74 (s, Aromatic-CH), 124.27 (s, Aromatic-CH), 121.40 (s, Aromatic-CH), 121.37 (s, Aromatic- $\mathrm{CH}$ ), 119.49 (s, Aromatic-C), 34.71 (s, CHCH3), $30.93\left(\mathrm{~s}, \mathrm{CHCH}_{3}\right), 25.21\left(\mathrm{~s}, \mathrm{CH}_{3}\right), 24.45\left(\mathrm{~s}, \mathrm{CH}_{3}\right), 24.42\left(\mathrm{~s}, \mathrm{CH}_{3}\right)$, and $20.88\left(\mathrm{~s}, \mathrm{CH}_{3}\right) \mathrm{ppm}$.

1(MesON). ${ }^{1} \mathrm{H}$ NMR (500 MHz, $\left.\mathrm{C}_{6} \mathrm{D}_{6}\right): \delta 10.44\left(\mathrm{~s}, 1 \mathrm{H}, \mathrm{W}=\mathrm{CH}, J_{\mathrm{CH}}=120 \mathrm{~Hz}\right), 8.75(\mathrm{~d}, 1 \mathrm{H}$, Aromatic-CH, $J=$ $5.9 \mathrm{~Hz}), 7.19(\mathrm{~d}, 1 \mathrm{H}$, Aromatic- $\mathrm{CH}, J=8.4 \mathrm{~Hz}), 7.07$ (br s, 1H, Aromatic- $\mathrm{CH}), 7.03-6.98(\mathrm{~m}, 2 \mathrm{H}$, Aromatic$\mathrm{CH}), 6.98-6.88(\mathrm{~m}, 6 \mathrm{H}$, Aromatic- $\mathrm{CH}), 6.82-6.77(\mathrm{~m}, 1 \mathrm{H}$, Aromatic- $\mathrm{CH}), 6.23-6.18(\mathrm{~m}, 1 \mathrm{H}$, Aromatic- $\mathrm{CH})$, $6.12(\mathrm{~s}, 2 \mathrm{H}, \mathrm{C}=\mathrm{CH}), 2.36\left(\mathrm{~s}, 3 \mathrm{H}, \mathrm{CH}_{3}\right), 2.29\left(\mathrm{br} \mathrm{s}, 6 \mathrm{H}, \mathrm{CH}_{3}\right), 2.13\left(\mathrm{~s}, 3 \mathrm{H}, \mathrm{CH}_{3}\right), 2.13\left(\mathrm{~s}, 3 \mathrm{H}, \mathrm{CH}_{3}\right), 1.96(\mathrm{~s}, 3 \mathrm{H}$, $\left.\mathrm{CH}_{3}\right), 1.90\left(\mathrm{~s}, 3 \mathrm{H}, \mathrm{CH}_{3}\right)$, and $1.49\left(\mathrm{~s}, 3 \mathrm{H}, \mathrm{CH}_{3}\right)$ ppm. ${ }^{13} \mathrm{C} \mathrm{NMR}\left(125 \mathrm{MHz}, \mathrm{C}_{6} \mathrm{D}_{6}\right): \delta 278.2(\mathrm{br} \mathrm{s}, \mathrm{W}=\mathrm{CH}), 155.2$ 
(s, Aromatic-C), 153.8 (s, Aromatic-C), 152.6 (s, Aromatic-CH), 150.0 (s, Aromatic-C), 138.4 (s, Aromatic$\mathrm{CH}), 136.5$ (s, Aromatic-C), 135.5 (s, Aromatic-CH), 134.9 (s, Aromatic-C), 134.2 (s, Aromatic-C), 133.9 (s, Aromatic-C), 130.3 (s, Aromatic-C), 129.5 (s, Aromatic-CH), 128.4 (s, Aromatic-CH), 127.8 (s, Aromatic$\mathrm{CH}), 127.0$ (s, Aromatic-CH), 126.4 (s, Aromatic- $\mathrm{CH}$ ), 126.0 (s, Aromatic- $\mathrm{CH}$ ), 123.1 (s, Aromatic- $\mathrm{CH}$ ), 122.1 (s, Aromatic-C), 121.2 (s, Aromatic- $\mathrm{CH}$ ), 108.6 (br s, $\mathrm{C}=\mathrm{CH}), 49.5\left(\mathrm{~s}, \mathrm{C}\left(\mathrm{CH}_{3}\right)_{2} \mathrm{Ph}\right), 33.0\left(\mathrm{~s}, \mathrm{CH}_{3}\right), 30.7$ $\left(\mathrm{s}, \mathrm{CH}_{3}\right), 21.4\left(\mathrm{~s}, \mathrm{CH}_{3}\right), 21.0\left(\mathrm{~s}, \mathrm{CH}_{3}\right), 20.9\left(\mathrm{~s}, \mathrm{CH}_{3}\right), 20.3\left(\mathrm{~s}, \mathrm{CH}_{3}\right)$, and $18.9\left(\mathrm{~s}, \mathrm{CH}_{3}\right) \mathrm{ppm}$.

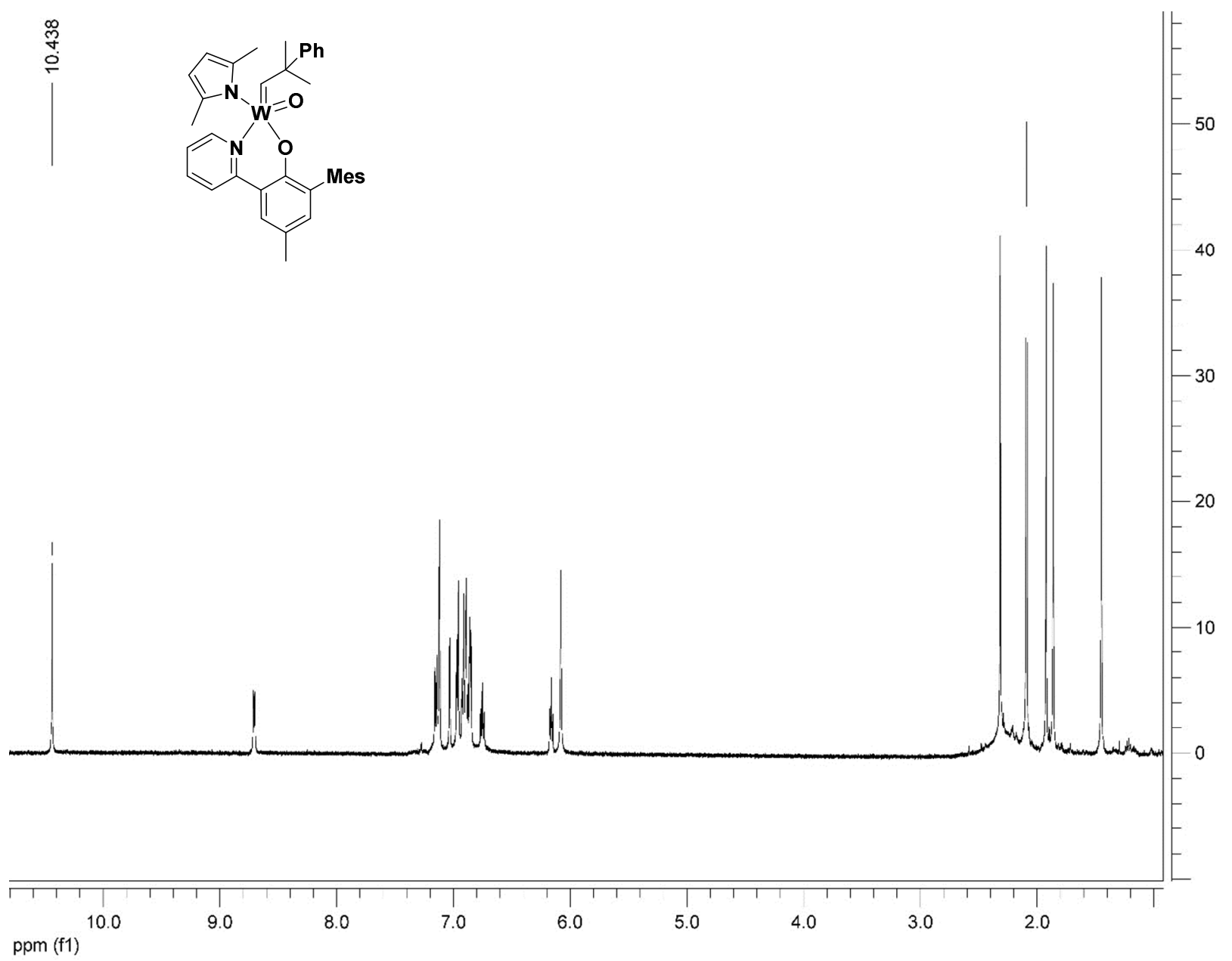

Figure S1. ${ }^{1} \mathrm{H}$ NMR (500 MHz, $\left.\mathrm{C}_{6} \mathrm{D}_{6}\right)$ spectrum and structure of $\mathbf{1}(\mathrm{MesON})$.

1(TripON). ${ }^{1} \mathrm{H}$ NMR (500 MHz, $\left.\mathrm{C}_{6} \mathrm{D}_{6}\right): \delta 10.62\left(\mathrm{~s}, 1 \mathrm{H}, \mathrm{W}=\mathrm{CH}, J_{\mathrm{CH}}=124 \mathrm{~Hz}\right), 9.03(\mathrm{~d}, 1 \mathrm{H}$, Aromatic-CH, $J=$ $5.3 \mathrm{~Hz}), 7.26(\mathrm{~d}, 1 \mathrm{H}$, Aromatic- $\mathrm{CH}, J=8.3 \mathrm{~Hz}), 7.19-7.11(\mathrm{~m}, 4 \mathrm{H}$, Aromatic- $\mathrm{CH}), 7.09-7.06(\mathrm{~m}, 2 \mathrm{H}$, Aromatic- $\mathrm{CH})$, 7.01-6.95 (m, 2H, Aromatic- $\mathrm{CH}), 6.94-6.89(\mathrm{~m}, 1 \mathrm{H}$, Aromatic- $\mathrm{CH}), 6.82-6.76(\mathrm{~m}, 1 \mathrm{H}$, Aromatic- $\mathrm{CH}), 6.27-6.22(\mathrm{~m}, 1 \mathrm{H}$, Aromatic- $\mathrm{CH}), 6.12(\mathrm{~s}, 2 \mathrm{H}, \mathrm{C}=\mathrm{CH}), 2.93\left(\mathrm{sept}, 1 \mathrm{H}, \mathrm{CH}\left(\mathrm{CH}_{3}\right)_{2}, J=6.9 \mathrm{~Hz}\right)$, 2.81 (sept, $1 \mathrm{H}, \mathrm{CH}\left(\mathrm{CH}_{3}\right)_{2}, J=6.9 \mathrm{~Hz}$ ), 2.73 (sept, $\left.\left.1 \mathrm{H}, \mathrm{CH}\left(\mathrm{CH}_{3}\right)_{2}, J=6.9 \mathrm{~Hz}\right), 2.28 \mathrm{br} \mathrm{s}, 6 \mathrm{H}, \mathrm{CH}_{3}\right), 2.14(\mathrm{~s}, 3 \mathrm{H}$, $\left.\mathrm{CH}_{3}\right), 1.86\left(\mathrm{~s}, 3 \mathrm{H}, \mathrm{CH}_{3}\right), 1.59\left(\mathrm{~s}, 3 \mathrm{H}, \mathrm{CH}_{3}\right), 1.41\left(\mathrm{~d}, 3 \mathrm{H}, \mathrm{CHCH}_{3}, J=6.9 \mathrm{~Hz}\right), 1.39\left(\mathrm{~d}, 3 \mathrm{H}, \mathrm{CHCH}_{3}, J=6.9 \mathrm{~Hz}\right)$, $1.25\left(\mathrm{~d}, 3 \mathrm{H}, \mathrm{CHCH}_{3}, J=6.9 \mathrm{~Hz}\right), 1.14\left(\mathrm{~d}, 3 \mathrm{H}, \mathrm{CHCH}_{3}, J=6.9 \mathrm{~Hz}\right), 1.12\left(\mathrm{~d}, 3 \mathrm{H}, \mathrm{CHCH}_{3}, J=6.9 \mathrm{~Hz}\right.$ ), and 1.02 (d, $3 \mathrm{H}, \mathrm{CHCH}_{3}, J=6.9 \mathrm{~Hz}$ ) ppm. ${ }^{13} \mathrm{C} \mathrm{NMR}\left(125 \mathrm{MHz}, \mathrm{C}_{6} \mathrm{D}_{6}\right): \delta 278.3$ (br s, W=CH), 155.3 (s, Aromatic-C), 
154.7 (s, Aromatic-C), 153.0 (s, Aromatic-CH), 150.4 (s, Aromatic-C), 148.6 (s, Aromatic-C), 147.2 (s, Aromatic-C), 146.0 (s, Aromatic-C), 138.4 (s, Aromatic-C), 136.7 (s, Aromatic-C), 135.4 (s, Aromatic-CH), 133.2 (s, Aromatic-C), 133.1 (s, Aromatic-C), 129.6 (s, Aromatic-C), 128.5 (s, Aromatic-CH), 127.3 (s, Aromatic-C), 126.4 (s, Aromatic-CH), 126.1 (s, Aromatic-CH), 122.9 (s, Aromatic-CH), 121.4 (s, Aromatic$\mathrm{CH}), 121.4$ (s, Aromatic-CH), 121.2 (s, Aromatic- $\mathrm{CH}), 120.5$ (s, Aromatic- $\mathrm{CH}), 108.4$ (br s, $\mathrm{C}=\mathrm{CH}), 49.6$ (s, $\left.\mathrm{C}\left(\mathrm{CH}_{3}\right)_{2} \mathrm{Ph}\right), 35.1\left(\mathrm{~s}, \mathrm{CHCH}_{3}\right), 33.1\left(\mathrm{~s}, \mathrm{CH}_{3}\right), 31.6\left(\mathrm{~s}, \mathrm{CH}_{3}\right), 31.0\left(\mathrm{~s}, \mathrm{CHCH}_{3}\right), 30.9\left(\mathrm{~s}, \mathrm{CHCH}_{3}\right), 25.7\left(\mathrm{~s}, \mathrm{CH}_{3}\right), 25.1$ $\left(\mathrm{s}, \mathrm{CH}_{3}\right), 24.7\left(\mathrm{~s}, \mathrm{CH}_{3}\right), 24.5\left(\mathrm{~s}, \mathrm{CH}_{3}\right), 23.8\left(\mathrm{~s}, \mathrm{CH}_{3}\right), 22.3\left(\mathrm{~s}, \mathrm{CH}_{3}\right), 20.9\left(\mathrm{~s}, \mathrm{CH}_{3}\right)$, and $18.8\left(\mathrm{~s}, \mathrm{CH}_{3}\right) \mathrm{ppm}$.

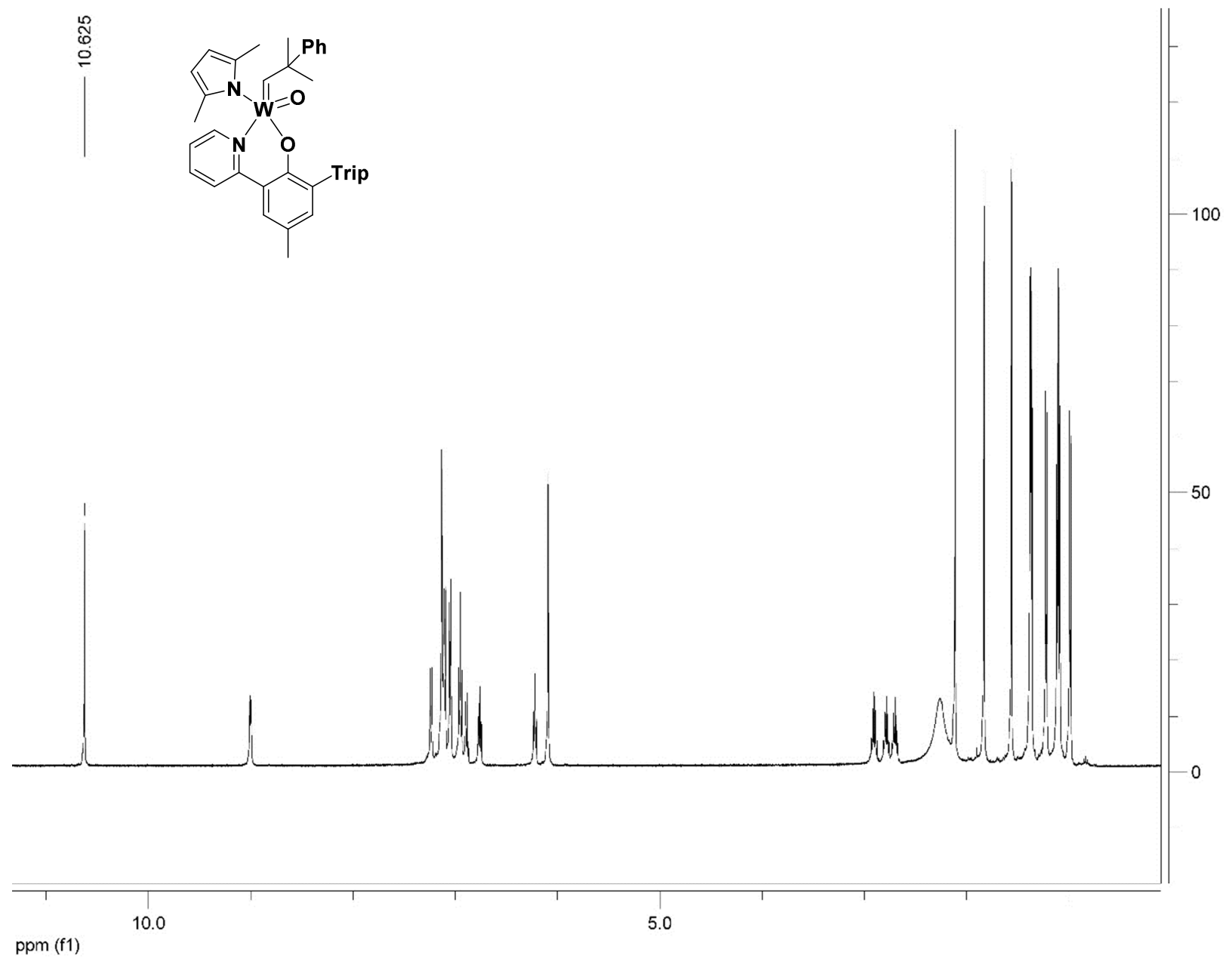

Figure S2. ${ }^{1} \mathrm{H}$ NMR (500 MHz, $\left.\mathrm{C}_{6} \mathrm{D}_{6}\right)$ spectrum and structure of $\mathbf{1}($ TripON).

2(MesON). ${ }^{1} \mathrm{H}$ NMR (500 MHz, $\left.\mathrm{C}_{6} \mathrm{D}_{6}\right) \delta: 14.48\left(\mathrm{~s}, \mathrm{Mo}=\mathrm{CH}, J_{\mathrm{CH}}=148 \mathrm{~Hz}\right.$, anti), $13.30\left(\mathrm{~s}, 1 \mathrm{H}, \mathrm{Mo}=\mathrm{CH}, J_{\mathrm{CH}}=\right.$ $127 \mathrm{~Hz}$, syn), 7.99 (dd, $1 \mathrm{H}$, Aromatic- $\mathrm{CH}, J=5.8,1.0 \mathrm{~Hz}$ ), 7.19-7.14 (m, 5H, Aromatic- $\mathrm{CH}$ ), 7.08-7.03 (m, $3 \mathrm{H}$, Aromatic- $\mathrm{CH}), 7.00-6.95(\mathrm{~m}, 1 \mathrm{H}$, Aromatic- $\mathrm{CH}), 6.93$ (br s, $1 \mathrm{H}$, Aromatic- $\mathrm{CH}), 6.77-6.72(\mathrm{~m}, 1 \mathrm{H}$, Aromatic- $\mathrm{CH}), 6.15-6.10(\mathrm{~m}, 1 \mathrm{H}$, Aromatic- $\mathrm{CH}), 2.65\left(\mathrm{~s}, 3 \mathrm{H}, \mathrm{CH}_{3}\right), 2.32\left(\mathrm{~s}, 3 \mathrm{H}, \mathrm{CH}_{3}\right), 2.24\left(\mathrm{~s}, 3 \mathrm{H}, \mathrm{CH}_{3}\right), 2.19$ $\left(\mathrm{s}, 3 \mathrm{H}, \mathrm{CH}_{3}\right), 2.15\left(\mathrm{~s}, 3 \mathrm{H}, \mathrm{CH}_{3}\right)$, and $1.41\left(\mathrm{~s}, 3 \mathrm{H}, \mathrm{CH}_{3}\right)$ ppm. $\left.{ }^{19} \mathrm{~F} \mathrm{NMR} \mathrm{(300} \mathrm{MHz,} \mathrm{C}_{6} \mathrm{D}_{6}\right) \delta:-146.81(\mathrm{~d}$, Aromatic-CF, $J=22.3 \mathrm{~Hz}$ ), $-158.43(\mathrm{t}$, Aromatic-CF, $J=21.7 \mathrm{~Hz}$ ), and $-163.23(\mathrm{t}$, Aromatic-CF, $J=21.7 \mathrm{~Hz}$ ) ppm. ${ }^{13} \mathrm{C}$ NMR (125 MHz, $\left.\mathrm{C}_{6} \mathrm{D}_{6}\right)$ ס: 306.22 (br s, Mo=CH), 155.65 (s, Aromatic-C), 153.50 (s, Aromatic-C), 152.53 (s, Aromatic-CH), 147.20 (s, Aromatic-C), 143.00 (dm, Aromatic-CF, J = 250 Hz), 139.06 (dm, 
Aromatic-CF, $J=250 \mathrm{~Hz}$ ), $138.87(\mathrm{~s}$, Aromatic-CH), $137.58(\mathrm{~s}$, Aromatic-C), 137.20 (dm, Aromatic-CF, $J=$ $250 \mathrm{~Hz}$ ), 136.68 (s, Aromatic-C), 136.51 (s, Aromatic-CH), 135.65 (s, Aromatic-C), 135.40 (s, Aromatic-C), 134.22 (s, Aromatic-C), 130.66 (s, Aromatic-C), 130.24-129.88 (m, Aromatic-C), 129.09 (s, Aromatic-C), 128.72 (s, Aromatic-CH), 127.47 (s, Aromatic-CH), 126.56 (s, Aromatic-CH), 126.48 (s, Aromatic-CH), 124.74 (s, Aromatic-CH), 123.25 (s, Aromatic-C), 120.59 (s, Aromatic- $\mathrm{CH}), 52.80\left(\mathrm{~s}, \mathrm{C}\left(\mathrm{CH}_{3}\right)_{2} \mathrm{Ph}\right), 30.03$ (s, $\left.\mathrm{CH}_{3}\right), 28.73\left(\mathrm{~s}, \mathrm{CH}_{3}\right), 22.22\left(\mathrm{~s}, \mathrm{CH}_{3}\right), 21.29\left(\mathrm{~s}, \mathrm{CH}_{3}\right), 21.05\left(\mathrm{~s}, \mathrm{CH}_{3}\right)$, and $20.84\left(\mathrm{~s}, \mathrm{CH}_{3}\right) \mathrm{ppm}$.

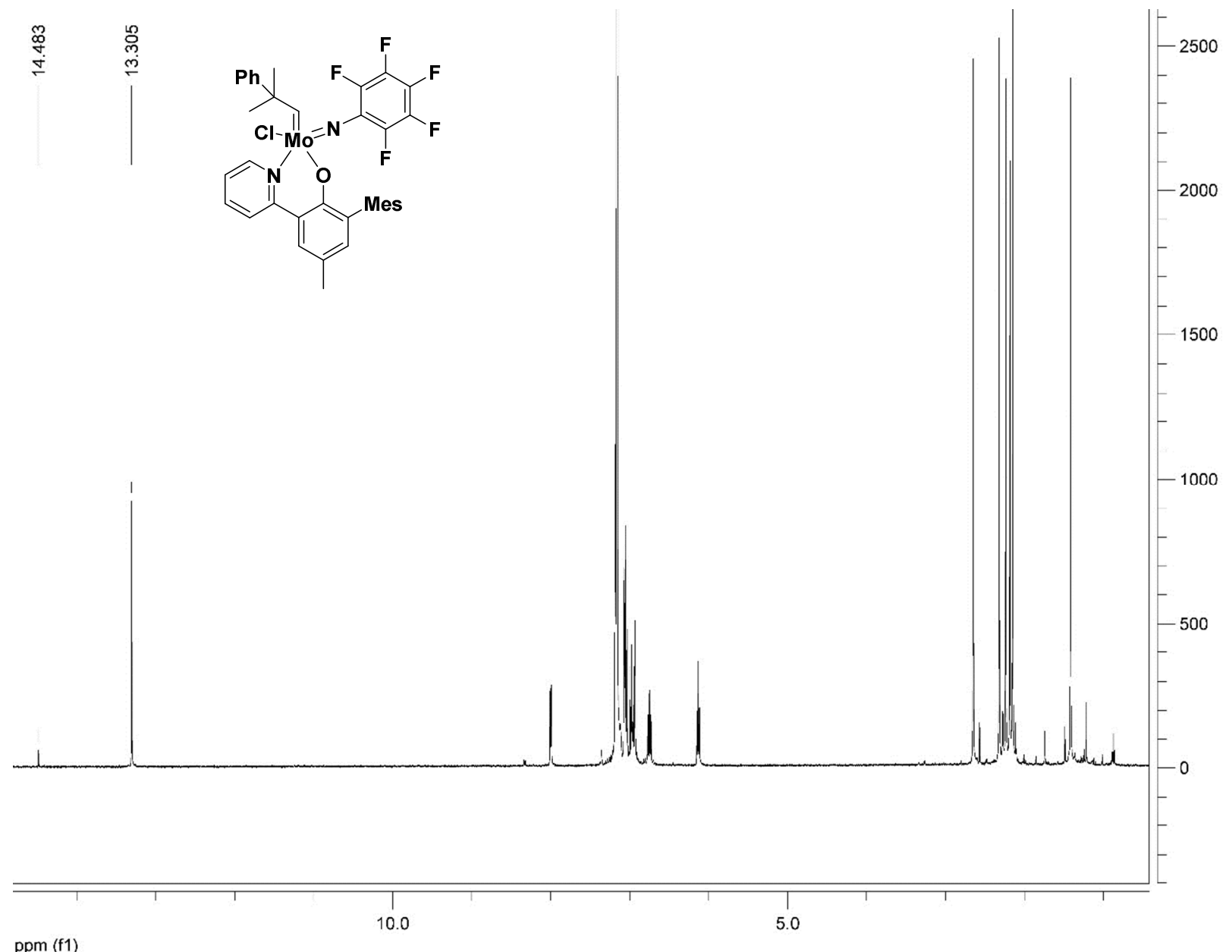

Figure S3. ${ }^{1} \mathrm{H}$ NMR $\left(500 \mathrm{MHz}, \mathrm{C}_{6} \mathrm{D}_{6}\right)$ spectrum and structure of $\mathbf{2}(\mathrm{MesON})$.

2(TripON). ${ }^{1} \mathrm{H}$ NMR (500 MHz, $\left.\mathrm{C}_{6} \mathrm{D}_{6}\right) \delta: 14.52\left(\mathrm{~s}, \mathrm{Mo}=\mathrm{CH}, J_{\mathrm{CH}}=147 \mathrm{~Hz}\right.$, anti), $13.39\left(\mathrm{~s}, 1 \mathrm{H}, \mathrm{Mo}=\mathrm{CH}, J_{\mathrm{CH}}=\right.$ $127 \mathrm{~Hz}, \mathrm{syn}$ ), 8.14 (dd, $1 \mathrm{H}$, Aromatic- $\mathrm{CH}, J=5.9,1.2 \mathrm{~Hz}), 7.42(\mathrm{~d}$, Aromatic- $\mathrm{CH}, J=1.7 \mathrm{~Hz}), 7.35-7.31(\mathrm{~m}$, $2 \mathrm{H}$, Aromatic- $\mathrm{CH}), 7.26-7.20(\mathrm{~m}, 4 \mathrm{H}$, Aromatic- $\mathrm{CH}), 7.08-7.02(\mathrm{~m}, 2 \mathrm{H}$, Aromatic- $\mathrm{CH}), 7.00-6.94(\mathrm{~m}, 1 \mathrm{H}$, Aromatic- $\mathrm{CH}), 6.77-6.71(\mathrm{~m}, 1 \mathrm{H}$, Aromatic- $\mathrm{CH}), 6.17-6.11(\mathrm{~m}, 1 \mathrm{H}$, Aromatic- $\mathrm{CH}), 3.16$ (sept, $1 \mathrm{H}$, $\mathrm{CH}\left(\mathrm{CH}_{3}\right)_{2}, J=6.8 \mathrm{~Hz}$ ), 3.12 (sept, $1 \mathrm{H}, \mathrm{CH}\left(\mathrm{CH}_{3}\right)_{2}, J=6.8 \mathrm{~Hz}$ ), 2.95 (sept, $\left.1 \mathrm{H}, \mathrm{CH}\left(\mathrm{CH}_{3}\right)_{2}, J=6.9 \mathrm{~Hz}\right), 2.18$ (s, $\left.3 \mathrm{H}, \mathrm{CH}_{3}\right), 2.17\left(\mathrm{~s}, 3 \mathrm{H}, \mathrm{CH}_{3}\right), 1.75\left(\mathrm{~d}, 3 \mathrm{H}, \mathrm{CHCH}_{3}, J=6.8 \mathrm{~Hz}\right), 1.57\left(\mathrm{~d}, 3 \mathrm{H}, \mathrm{CHCH}_{3}, J=6.8 \mathrm{~Hz}\right), 1.55(\mathrm{~s}, 3 \mathrm{H}$, $\left.\mathrm{CH}_{3}\right), 1.37\left(\mathrm{~d}, 3 \mathrm{H}, \mathrm{CHCH}_{3}, J=6.8 \mathrm{~Hz}\right), 1.34\left(\mathrm{~d}, 3 \mathrm{H}, \mathrm{CHCH}_{3}, J=6.9 \mathrm{~Hz}\right), 1.33\left(\mathrm{~d}, 3 \mathrm{H}, \mathrm{CHCH}_{3}, J=6.9 \mathrm{~Hz}\right)$, and $1.24\left(\mathrm{~d}, 3 \mathrm{H}, \mathrm{CHCH}_{3}, J=6.8 \mathrm{~Hz}\right) \mathrm{ppm} .{ }^{19} \mathrm{~F} \mathrm{NMR}\left(300 \mathrm{MHz}, \mathrm{C}_{6} \mathrm{D}_{6}\right) \delta:-146.52$ (d, Aromatic-CF, $\left.J=18.8 \mathrm{~Hz}\right),-$ 
158.00 (t, Aromatic-CF, $J=21.5 \mathrm{~Hz}$ ), and -163.10-164.42 (m, Aromatic-CF) ppm. ${ }^{13} \mathrm{C}$ NMR (125 MHz, $\mathrm{C}_{6} \mathrm{D}_{6}$ ) $\delta: 306.17$ (br s, Mo=CH), 155.87 (s, Aromatic-C), 154.64 (s, Aromatic-C), 152.63 (s, Aromatic- $\mathrm{CH}$ ), 148.56 (s, Aromatic-C), 147.88 (s, Aromatic-C), 147.53 (s, Aromatic-C), 146.81 (s, Aromatic-C), 143.03 (dm, Aromatic-CF, $J=250 \mathrm{~Hz}$ ), $139.21(\mathrm{dm}$, Aromatic-CF, $J=250 \mathrm{~Hz}), 138.76$ (s, Aromatic-CH), 137.56 (s, Aromatic-CH), 137.26 (dm, Aromatic-CF, J = $250 \mathrm{~Hz}$ ), 133.56 (s, Aromatic-C), 133.38 (s, Aromatic-C), 130.02-129.88 (m, Aromatic-C), 129.83 (s, Aromatic-C), 128.78 (s, Aromatic-CH), 127.46 (s, Aromatic$\mathrm{CH}), 126.55$ (s, Aromatic-CH), 126.52 (s, Aromatic-CH), 124.69 (s, Aromatic-CH), 122.21 (s, Aromatic-C), 121.44 (s, Aromatic-CH), 120.60 (s, Aromati-CH), 120.54 (s, Aromatic- $\mathrm{CH}), 52.86$ (s, $\left.\mathrm{C}\left(\mathrm{CH}_{3}\right)_{2} \mathrm{Ph}\right), 34.97$ (s, $\mathrm{CHCH}_{3}$ ), $31.72\left(\mathrm{~s}, \mathrm{CHCH}_{3}\right), 31.67\left(\mathrm{~s}, \mathrm{CHCH}_{3}\right), 30.39\left(\mathrm{~s}, \mathrm{CH}_{3}\right), 29.05\left(\mathrm{~s}, \mathrm{CH}_{3}\right), 26.11\left(\mathrm{~s}, \mathrm{CH}_{3}\right), 25.39\left(\mathrm{~s}, \mathrm{CH}_{3}\right)$, $24.61\left(\mathrm{~s}, \mathrm{CH}_{3}\right), 24.43\left(\mathrm{~s}, \mathrm{CH}_{3}\right), 24.40\left(\mathrm{~s}, \mathrm{CH}_{3}\right), 24.24\left(\mathrm{~s}, \mathrm{CH}_{3}\right)$, and $20.96\left(\mathrm{~s}, \mathrm{CH}_{3}\right) \mathrm{ppm}$.

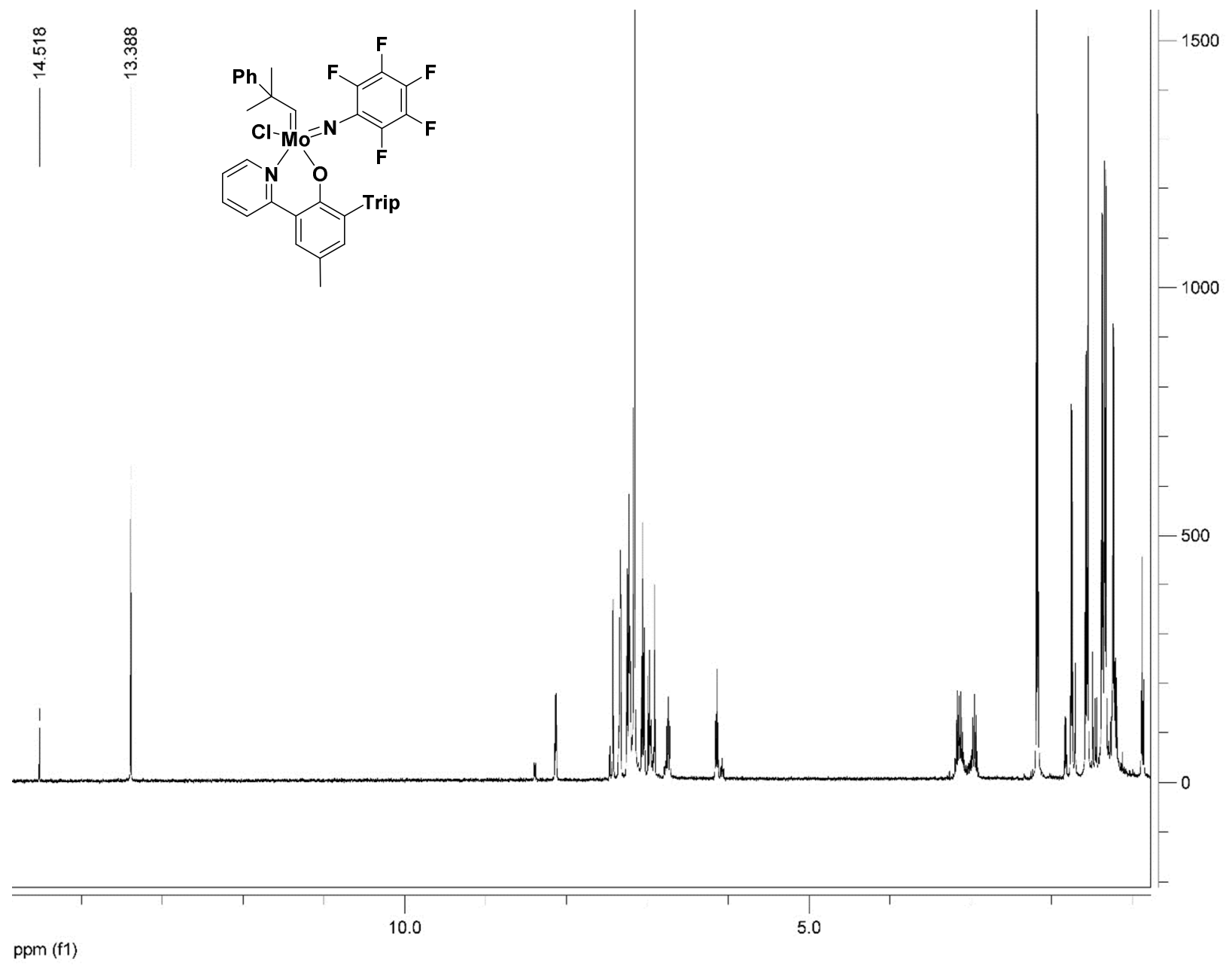

Figure S4. ${ }^{1} \mathrm{H} N M R\left(500 \mathrm{MHz}, \mathrm{C}_{6} \mathrm{D}_{6}\right)$ spectrum and structure of 2(TripON).

3(MesON). ${ }^{1} \mathrm{H}$ NMR (500 MHz, $\left.\mathrm{C}_{6} \mathrm{D}_{6}\right) \delta: 14.32\left(\mathrm{~s}, 1 \mathrm{H}, \mathrm{Mo}=\mathrm{CH}, J_{\mathrm{CH}}=146 \mathrm{~Hz}\right), 8.38(\mathrm{~d}, 1 \mathrm{H}$, Aromatic-CH, $J=$ $5.0 \mathrm{~Hz}), 7.21-7.13(\mathrm{~m}, 5 \mathrm{H}$, Aromatic-CH), $7.10(\mathrm{~s}, 1 \mathrm{H}$, Aromatic- $\mathrm{CH}), 7.06(\mathrm{~s}, 1 \mathrm{H}$, Aromatic- $\mathrm{CH}), 7.01-6.92$ $(\mathrm{m}, 3 \mathrm{H}$, Aromatic- $\mathrm{CH}), 6.67(\mathrm{t}, 1 \mathrm{H}$, Aromatic- $\mathrm{CH}, J=7.4 \mathrm{~Hz}), 6.63(\mathrm{~s}, 1 \mathrm{H}$, Aromatic-CH), $6.62(\mathrm{~s}, 1 \mathrm{H}$, Aromatic- $\mathrm{CH}), 6.57(\mathrm{dd}, 1 \mathrm{H}$, Aromatic- $\mathrm{CH}, J=8.2,6.6 \mathrm{~Hz}), 5.97(\mathrm{t}, 1 \mathrm{H}$, Aromatic- $\mathrm{CH}, J=6.4 \mathrm{~Hz}), 2.50(\mathrm{~s}$, 
$\left.3 \mathrm{H}, \mathrm{CH}_{3}\right), 2.35\left(\mathrm{~s}, 3 \mathrm{H}, \mathrm{CH}_{3}\right), 2.29\left(\mathrm{~s}, 6 \mathrm{H}, \mathrm{CH}_{3}\right), 2.27\left(\mathrm{~s}, 3 \mathrm{H}, \mathrm{CH}_{3}\right), 2.21\left(\mathrm{~s}, 3 \mathrm{H}, \mathrm{CH}_{3}\right), 1.83\left(\mathrm{~s}, 3 \mathrm{H}, \mathrm{CH}_{3}\right)$, and

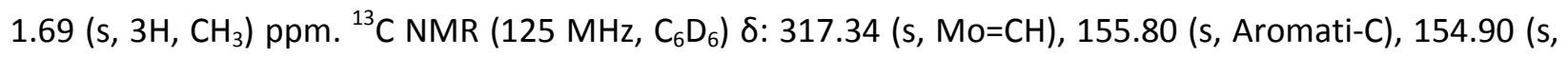
Aromatic-C), 154.82 (s, Aromatic-CH), 149.72 (s, Aromatic-C), 138.03 (s, Aromatic-C), 136.29 (s, Aromatic-C), 135.58 (s, Aromatic-C), , 138.14 (s, Aromatic-CH), 135.94 (s, Aromatic-CH), 135.45 (s, Aromatic-C), 135.25 (s, Aromatic-C), 128.77 (s, Aromatic-CH), 128.66 (s, Aromatic-C), 128.35 (s, Aromatic-CH), 127.82 (s, Aromatic-CH), 127.64 (s, Aromatic-CH), 126.60 (s, Aromatic-CH), 126.19 (s, Aromatic-CH), 125.96 (s, Aromatic-CH), 124.30 (s, Aromatic-CH), 122.83 (s, Aromatic-C), 120.14 (s, Aromatic- $\mathrm{CH}$ ), 119.14 (s, Aromatic- $\mathrm{C}$ ), 51.36 (s, $\left.\mathrm{C}\left(\mathrm{CH}_{3}\right)_{2} \mathrm{Ph}\right), 28.71\left(\mathrm{~s}, \mathrm{CH}_{3}\right), 27.08\left(\mathrm{~s}, \mathrm{CH}_{3}\right), 21.53\left(\mathrm{~s}, \mathrm{CH}_{3}\right)$, $21.23\left(\mathrm{~s}, \mathrm{CH}_{3}\right), 20.79\left(\mathrm{~s}, \mathrm{CH}_{3}\right), 20.78\left(\mathrm{~s}, \mathrm{CH}_{3}\right)$, and $19.32\left(\mathrm{~s}, \mathrm{CH}_{3}\right) \mathrm{ppm}$.

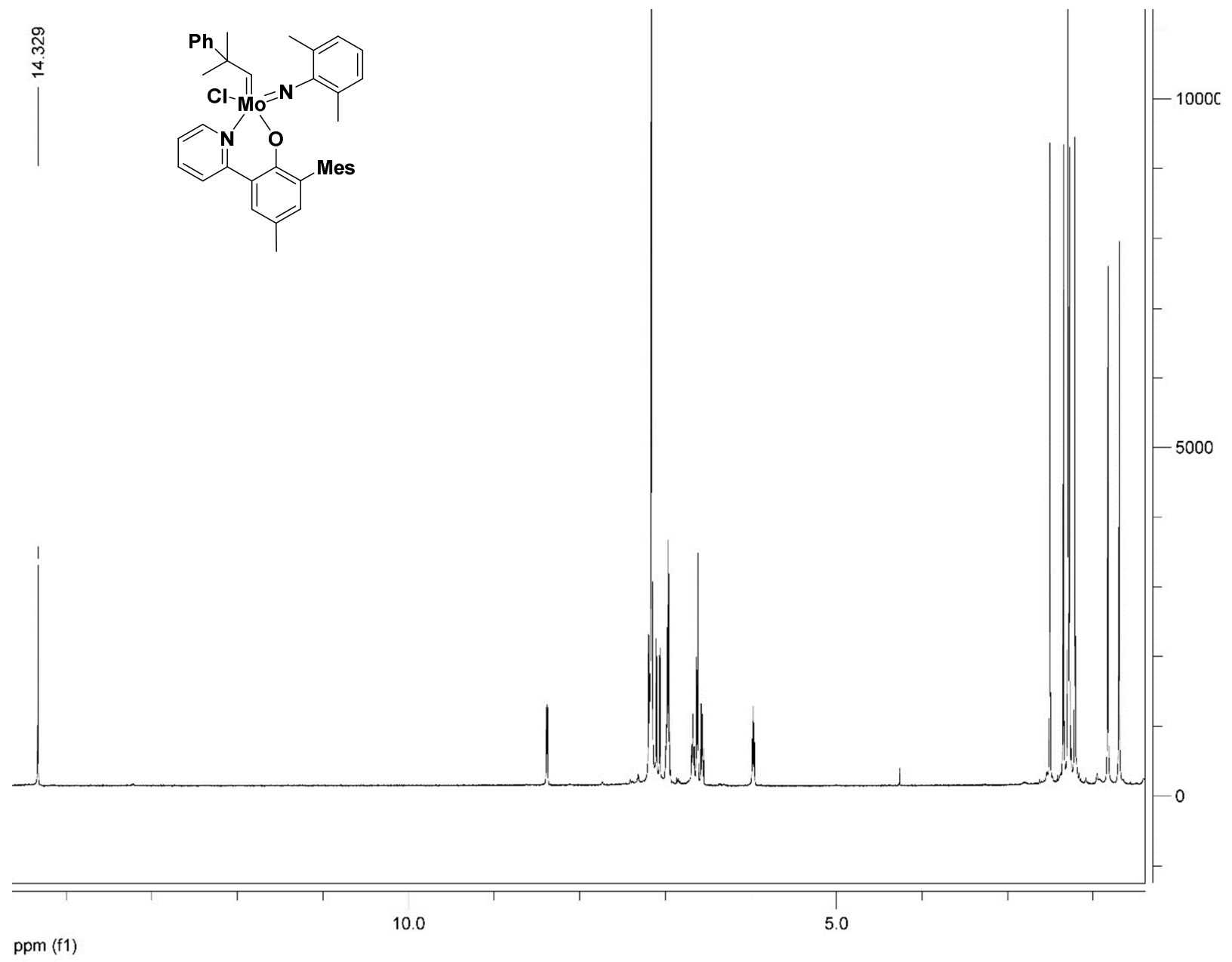

Figure S5. ${ }^{1} \mathrm{H} N M R\left(500 \mathrm{MHz}, \mathrm{C}_{6} \mathrm{D}_{6}\right)$ spectrum and structure of $\mathbf{3}(\mathrm{MesON})$.

3(TripON). ${ }^{1} \mathrm{H}$ NMR $\left(500 \mathrm{MHz}, \mathrm{C}_{6} \mathrm{D}_{6}\right) \delta: 14.35\left(\mathrm{~s}, 1 \mathrm{H}, \mathrm{Mo}=\mathrm{CH}, J_{\mathrm{CH}}=146 \mathrm{~Hz}\right), 8.40$ (dd, $1 \mathrm{H}$, Aromatic-CH, $J=$ 7.1, $5.9 \mathrm{~Hz}), 7.44(\mathrm{~d}, 1 \mathrm{H}$, Aromatic- $\mathrm{CH}, J=1.6 \mathrm{~Hz}), 7.36(\mathrm{~d}, 1 \mathrm{H}$, Aromatic- $\mathrm{CH}, J=1.6 \mathrm{~Hz}), 7.32(\mathrm{~s}, 1 \mathrm{H}$, Aromatic- $\mathrm{CH}, \mathrm{J}=2.1 \mathrm{~Hz}), 7.22-7.17(\mathrm{~m}, 3 \mathrm{H}$, Aromatic- $\mathrm{CH}), 7.13-7.09(\mathrm{~m}, 3 \mathrm{H}$, Aromatic- $\mathrm{CH}), 7.00-6.94(\mathrm{~m}$, $3 \mathrm{H}$, Aromatic- $\mathrm{CH}), 6.70-6.63(\mathrm{~m}, 3 \mathrm{H}$, Aromatic- $\mathrm{CH}), 6.59(\mathrm{dd}, 1 \mathrm{H}$, Aromatic- $\mathrm{CH}, J=8.2,6.7 \mathrm{~Hz}), 5.93(\mathrm{t}$, $1 \mathrm{H}$, Aromatic- $\mathrm{CH}, J=6.1 \mathrm{~Hz}$ ), $3.14\left(\right.$ sept, $\left.1 \mathrm{H}, \mathrm{CH}\left(\mathrm{CH}_{3}\right)_{2}, J=6.8 \mathrm{~Hz}\right), 3.00$ (sept, $1 \mathrm{H}, \mathrm{CH}\left(\mathrm{CH}_{3}\right)_{2}, J=6.9 \mathrm{~Hz}$ ), 
2.98 (sept, $\left.1 \mathrm{H}, \mathrm{CH}\left(\mathrm{CH}_{3}\right)_{2}, J=6.9 \mathrm{~Hz}\right), 2.34\left(\mathrm{~s}, 6 \mathrm{H}, \mathrm{CH}_{3}\right), 2.23\left(\mathrm{~s}, 3 \mathrm{H}, \mathrm{CH}_{3}\right), 1.79\left(\mathrm{~s}, 3 \mathrm{H}, \mathrm{CH}_{3}\right), 1.74(\mathrm{~s}, 3 \mathrm{H}$, $\left.\mathrm{CHCH}_{3}, J=6.9 \mathrm{~Hz}\right), 1.69\left(\mathrm{~s}, 3 \mathrm{H}, \mathrm{CH}_{3}\right), 1.47\left(\mathrm{~s}, 3 \mathrm{H}, \mathrm{CHCH}_{3}, J=6.9 \mathrm{~Hz}\right), 1.38-1.34\left(\mathrm{~m}, 9 \mathrm{H}, \mathrm{CHCH}_{3}\right)$, and 1.21 $\left(\mathrm{d}, 3 \mathrm{H}, \mathrm{CHCH}_{3}, J=6.8 \mathrm{~Hz}\right.$ ) ppm. ${ }^{13} \mathrm{C} \mathrm{NMR}\left(125 \mathrm{MHz}, \mathrm{C}_{6} \mathrm{D}_{6}\right) \delta: 317.81$ (s, Mo=CH), 155.80 (s, Aromatic-C), 154.90 (s, Aromatic-C), 154.82 (s, Aromatic-CH), 149.72 (s, Aromatic-C), 138.03 (s, Aromatic-C), 136.29 (s, Aromatic-C), 135.58 (s, Aromatic-C), 128.14 (s, Aromatic-CH), 127.82 (s, Aromatic-CH), 127.64 (s, Aromatic-CH), 126.60 (s, Aromatic-CH), 126.19 (s, Aromatic-CH), 125.96 (s, Aromatic-CH), 124.30 (s, Aromatic-CH), 122.83 (s, Aromatic-C), 120.14 (s, Aromatic-CH), 119.14 (s, Aromatic-C), 51.51 (s, $\left.\mathrm{C}\left(\mathrm{CH}_{3}\right)_{2} \mathrm{Ph}\right), 34.97$ (s, $\left.\mathrm{CHCH}_{3}\right), 31.59$ (s, $\left.\mathrm{CHCH}_{3}\right), 31.27$ (s, $\left.\mathrm{CHCH}_{3}\right), 29.12$ (s, $\left.\mathrm{CH}_{3}\right), 27.27$ (s, $\left.\mathrm{CH}_{3}\right), 26.55$ (s, $\left.\mathrm{CH}_{3}\right), 25.90\left(\mathrm{~s}, \mathrm{CH}_{3}\right), 24.70\left(\mathrm{~s}, \mathrm{CH}_{3}\right), 24.44\left(\mathrm{~s}, \mathrm{CH}_{3}\right), 23.88\left(\mathrm{~s}, \mathrm{CH}_{3}\right), 23.35\left(\mathrm{~s}, \mathrm{CH}_{3}\right), 20.94\left(\mathrm{~s}, \mathrm{CH}_{3}\right)$, and 19.37 $\left(\mathrm{s}, \mathrm{CH}_{3}\right) \mathrm{ppm}$.

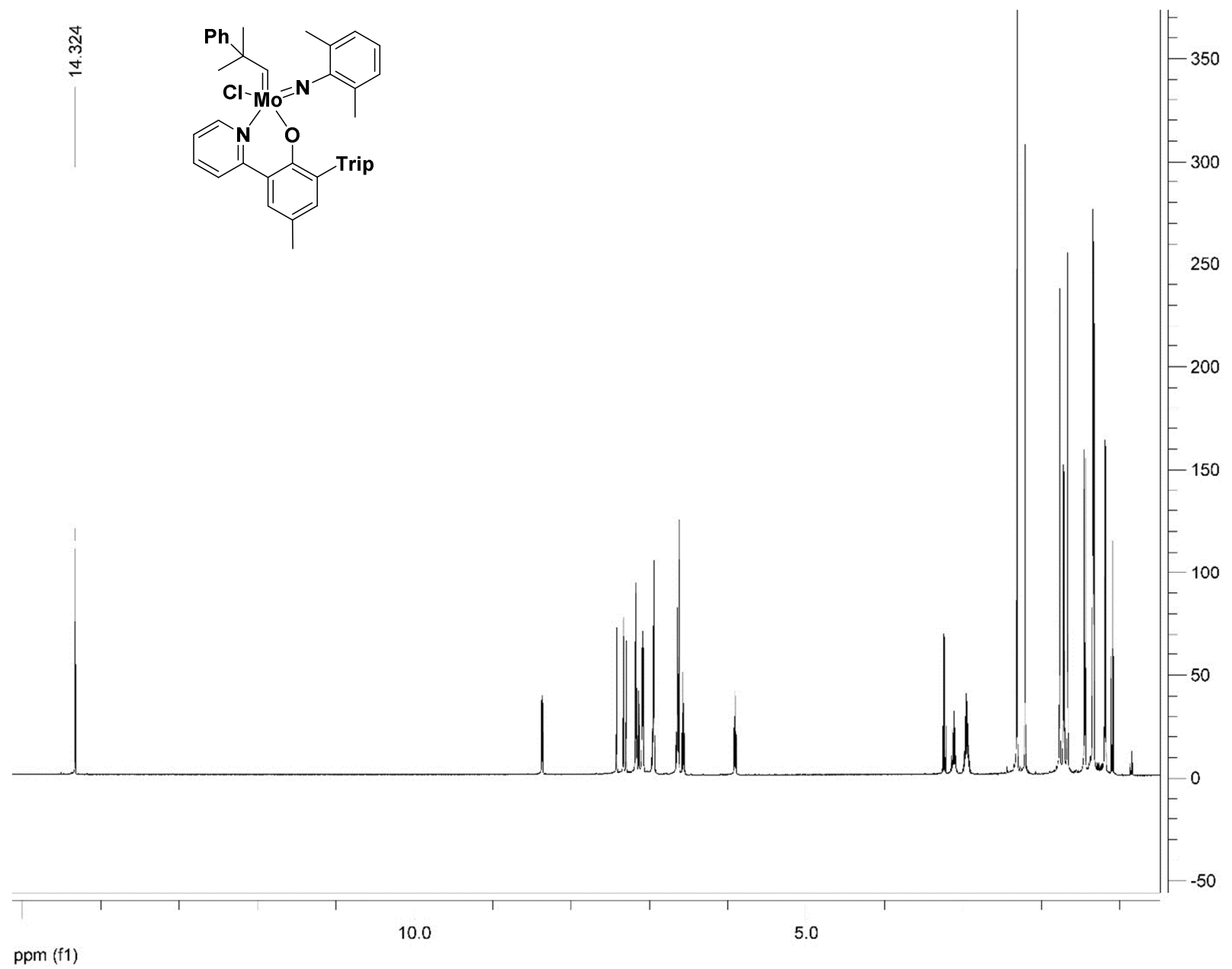

Figure S6. ${ }^{1} \mathrm{H} N M R\left(500 \mathrm{MHz}, \mathrm{C}_{6} \mathrm{D}_{6}\right)$ spectrum and structure of 3(TripON).

4(MesON). ${ }^{1} \mathrm{H}$ NMR (500 MHz, $\left.\mathrm{C}_{6} \mathrm{D}_{6}\right) \delta: 13.30\left(\mathrm{~s}, 1 \mathrm{H}, \mathrm{Mo}=\mathrm{CH}, J_{\mathrm{CH}}=125 \mathrm{~Hz}\right), 7.96(\mathrm{~d}, 1 \mathrm{H}$, Aromatic-CH, $J=$ $5.2 \mathrm{~Hz}), 7.21(\mathrm{~s}, 1 \mathrm{H}$, Aromatic- $\mathrm{CH}), 7.19(\mathrm{~s}, 1 \mathrm{H}$, Aromatic- $\mathrm{CH}), 7.13-7.05(\mathrm{~m}, 4 \mathrm{H}$, Aromatic- $\mathrm{CH}), 7.00(\mathrm{t}, 1 \mathrm{H}$, Aromatic- $\mathrm{CH}, J=7.4 \mathrm{~Hz}), 6.96(\mathrm{~s}, 1 \mathrm{H}$, Aromatic- $\mathrm{CH}), 6.86(\mathrm{~d}, 1 \mathrm{H}$, Aromatic- $\mathrm{CH}, J=7.8 \mathrm{~Hz}), 6.82(\mathrm{t}, 1 \mathrm{H}$, Aromatic- $\mathrm{CH}, J=7.4 \mathrm{~Hz}), 6.77(\mathrm{t}, 1 \mathrm{H}$, Aromatic- $\mathrm{CH}, J=7.3 \mathrm{~Hz}), 6.08(\mathrm{t}, 1 \mathrm{H}$, Aromatic- $\mathrm{CH}, J=6.4 \mathrm{~Hz}), 2.64$ 
$\left(\mathrm{s}, 3 \mathrm{H}, \mathrm{CH}_{3}\right), 2.38\left(\mathrm{~s}, 3 \mathrm{H}, \mathrm{CH}_{3}\right), 2.33\left(\mathrm{~s}, 6 \mathrm{H}, \mathrm{CH}_{3}\right), 2.26\left(\mathrm{~s}, 3 \mathrm{H}, \mathrm{CH}_{3}\right), 2.23\left(\mathrm{~s}, 3 \mathrm{H}, \mathrm{CH}_{3}\right), 2.19\left(\mathrm{~s}, 3 \mathrm{H}, \mathrm{CH}_{3}\right), 1.95$ $\left(\mathrm{s}, 3 \mathrm{H}, \mathrm{CH}_{3}\right), 1.47\left(\mathrm{~s}, 3 \mathrm{H}, \mathrm{CH}_{3}\right)$ and $1.05\left(\mathrm{~s}, 9 \mathrm{H}, \mathrm{C}\left(\mathrm{CH}_{3}\right)_{3}\right)$ ppm. $\left.{ }^{13} \mathrm{C} \mathrm{NMR} \mathrm{(125} \mathrm{MHz,} \mathrm{C}_{6} \mathrm{D}_{6}\right)$ ס: 301.62 (s, $\mathrm{Mo}=\mathrm{CH}$ ), 156.53 (s, Aromatic-C), 155.19 (s, Aromatic-C), 153.69 (s, Aromatic-CH), 148.40 (s, Aromatic-C), 138.37 (s, Aromatic-CH), 137.45 (s, Aromatic-C), 136.54 (s, Aromatic-C), 136.29 (s, Aromatic-C), 136.04 (s, Aromatic-C), 135.04 (s, Aromatic-C), 129.56 (s, Aromatic- $\mathrm{CH}$ ), 128.96 (s, Aromatic-C), 128.59 (s, Aromatic-CH), 128.47 (s, Aromatic-C), 127.16 (s, Aromatic-CH), 126.64 (s, Aromatic-CH), 126.17 (s, Aromatic-CH), 124.39 (s, Aromatic- $\mathrm{CH}$ ), 122.48 (s, Aromatic-C), 121.16 (s, Aromatic- $\mathrm{CH}$ ), 119.68 (s, Aromatic- $\mathrm{CH}$ ), 70.00 (s, $\left.\mathrm{C}\left(\mathrm{CH}_{3}\right)_{3}\right), 51.28\left(\mathrm{~s}, \mathrm{C}\left(\mathrm{CH}_{3}\right)_{2} \mathrm{Ph}\right), 30.90\left(\mathrm{~s}, \mathrm{CH}_{3}\right), 29.88\left(\mathrm{~s}, \mathrm{C}\left(\mathrm{CH}_{3}\right)_{3}\right), 29.61\left(\mathrm{~s}, \mathrm{CH}_{3}\right)$, $22.47\left(\mathrm{~s}, \mathrm{CH}_{3}\right), 21.37\left(\mathrm{~s}, \mathrm{CH}_{3}\right), 21.11\left(\mathrm{~s}, \mathrm{CH}_{3}\right)$, and $20.91\left(\mathrm{~s}, \mathrm{CH}_{3}\right) \mathrm{ppm}$.

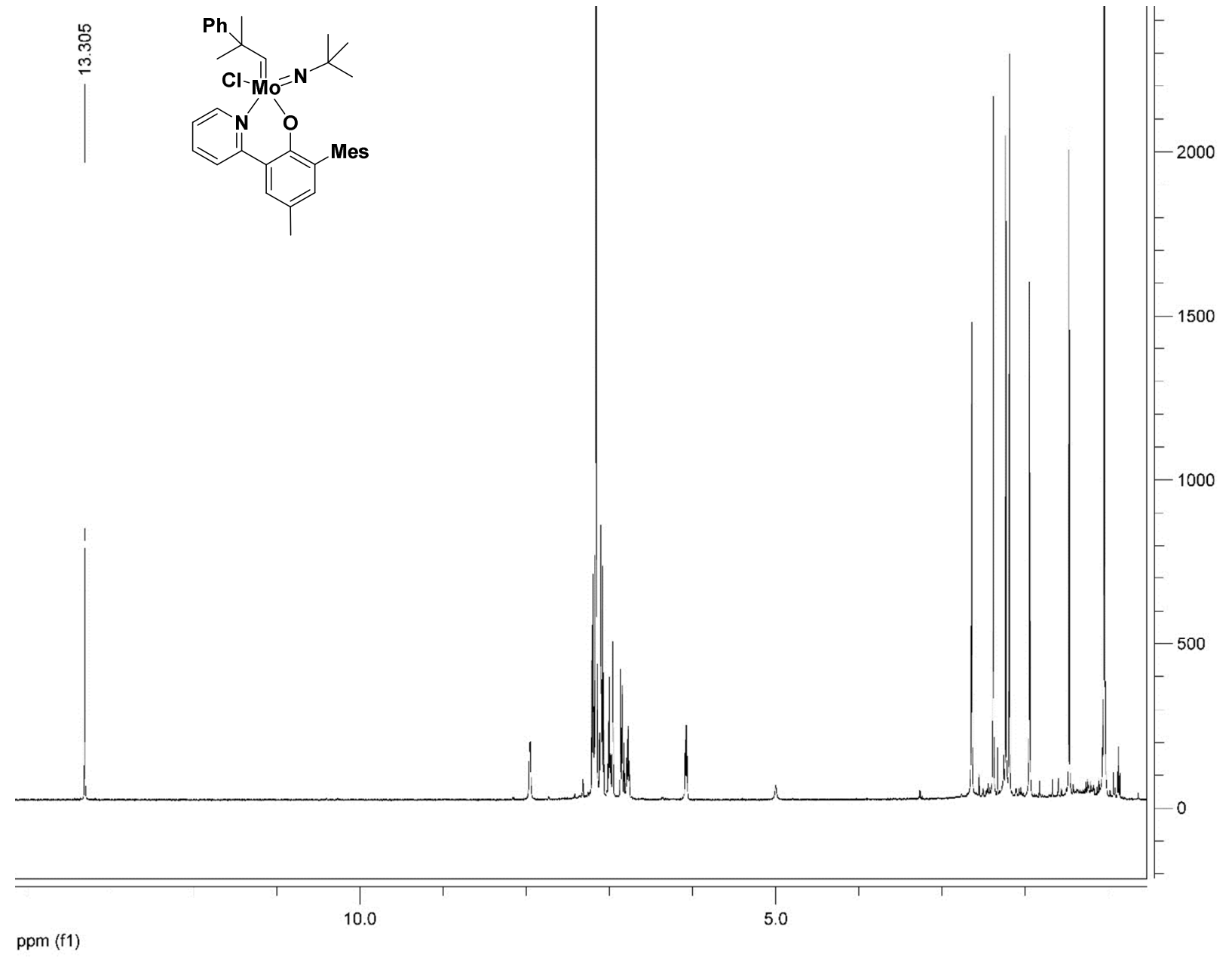

Figure S7. ${ }^{1} \mathrm{H}$ NMR (500 MHz, $\left.\mathrm{C}_{6} \mathrm{D}_{6}\right)$ spectrum and structure of $\mathbf{4}(\mathrm{MesON})$.

4(TripON). ${ }^{1} \mathrm{H}$ NMR (500 MHz, $\left.\mathrm{C}_{6} \mathrm{D}_{6}\right) \delta: 13.31\left(\mathrm{~s}, 1 \mathrm{H}, \mathrm{Mo}=\mathrm{CH}, J_{\mathrm{CH}}=125 \mathrm{~Hz}\right), 8.22(\mathrm{~d}, 1 \mathrm{H}$, Aromatic- $\mathrm{CH}, J=$ $5.6 \mathrm{~Hz}), 7.41(\mathrm{~s}, 1 \mathrm{H}$, Aromatic- $\mathrm{CH}), 7.35-7.30(\mathrm{~m}, 2 \mathrm{H}$, Aromatic- $\mathrm{CH}), 7.26-7.21(\mathrm{~m}, 3 \mathrm{H}$, Aromatic- $\mathrm{CH}), 7.20$ $(\mathrm{s}, 1 \mathrm{H}$, Aromatic-CH), $7.07(\mathrm{t}, 2 \mathrm{H}$, Aromatic- $\mathrm{CH}, \mathrm{J}=7.7 \mathrm{~Hz}), 6.99(\mathrm{t}, 1 \mathrm{H}$, Aromatic- $\mathrm{CH}, \mathrm{J}=7.2 \mathrm{~Hz}), 6.81-6.75$ (m, $1 \mathrm{H}$, Aromatic- $\mathrm{CH}), 6.14-6.8(\mathrm{~m}, 1 \mathrm{H}$, Aromatic- $\mathrm{CH}), 3.19$ (sept, $1 \mathrm{H}, \mathrm{CH}\left(\mathrm{CH}_{3}\right)_{2}, \mathrm{~J}=6.9 \mathrm{~Hz}$ ), 3.16 (sept, $1 \mathrm{H}$, $\left.\mathrm{CH}\left(\mathrm{CH}_{3}\right)_{2}, J=6.9 \mathrm{~Hz}\right), 2.96$ (sept, $\left.1 \mathrm{H}, \mathrm{CH}\left(\mathrm{CH}_{3}\right)_{2}, J=6.8 \mathrm{~Hz}\right), 2.22\left(\mathrm{~s}, 3 \mathrm{H}, \mathrm{CH}_{3}\right), 1.89\left(\mathrm{~s}, 3 \mathrm{H}, \mathrm{CH}_{3}\right), 1.72(\mathrm{~s}, 3 \mathrm{H}$, 
$\left.\mathrm{CHCH}_{3}, J=6.8 \mathrm{~Hz}\right), 1.58\left(\mathrm{~s}, 3 \mathrm{H}, \mathrm{CHCH}_{3}, J=6.9 \mathrm{~Hz}\right), 1.54\left(\mathrm{~s}, 3 \mathrm{H}, \mathrm{CH}_{3}\right), 1.37\left(\mathrm{~d}, 3 \mathrm{H}, \mathrm{CHCH}_{3}, J=6.8 \mathrm{~Hz}\right), 1.35$ $\left(\mathrm{d}, 3 \mathrm{H}, \mathrm{CHCH}_{3}, J=6.9 \mathrm{~Hz}\right), 1.34\left(\mathrm{~d}, 3 \mathrm{H}, \mathrm{CHCH}_{3}, J=6.9 \mathrm{~Hz}\right), 1.24\left(\mathrm{~d}, 3 \mathrm{H}, \mathrm{CHCH}_{3}\right)$, and $1.13\left(\mathrm{~s}, 9 \mathrm{H}, \mathrm{C}\left(\mathrm{CH}_{3}\right)_{3}\right)$ ppm. ${ }^{13} \mathrm{C}$ NMR (125 MHz, $\mathrm{C}_{6} \mathrm{D}_{6}$ ) $\delta: 306.21$ (s, Mo=CH), 156.95 (s, Aromatic-C), 156.55 (s, Aromatic-C), 154.09 (s, Aromatic-CH), 149.00 (s, Aromatic-C), 148.73 (s, Aromatic-C), 148.07 (s, Aromatic-C), 147.27 (s, Aromatic-CH), 147.06 (s, Aromatic-CH), 138.32 (s, Aromatic-C), 137.43 (s, Aromatic-CH), 134.39 (s, Aromatic-C), 134.17 (s, Aromatic-C), 128.59 (s, Aromatic-CH), 127.57 (s, Aromatic-CH), 127.39 (s, Aromatic-CH), 126.67 (s, Aromatic-CH), 126.21 (s, Aromatic- $\mathrm{CH}$ ), 124.46 (s, Aromatic-CH), 122.31 (s, Aromatic-C), 121.07 (s, Aromatic- $\mathrm{CH}$ ), 120.58 (s, Aromatic- $\mathrm{CH}$ ), 119.36 (s, Aromatic-C), $70.04\left(\mathrm{~s}, \mathrm{C}\left(\mathrm{CH}_{3}\right)_{3}\right)$, 51.51 (s, $\left.\mathrm{C}\left(\mathrm{CH}_{3}\right)_{2} \mathrm{Ph}\right), 35.01$ (s, $\left.\mathrm{CHCH}_{3}\right), 31.68\left(\mathrm{~s}, \mathrm{CHCH}_{3}\right), 31.65\left(\mathrm{~s}, \mathrm{CHCH}_{3}\right), 31.12\left(\mathrm{~s}, \mathrm{CH}_{3}\right), 30.07\left(\mathrm{~s}, \mathrm{CH}_{3}\right)$, $29.80\left(\mathrm{~s}, \mathrm{CH}_{3}\right), 26.07\left(\mathrm{~s}, \mathrm{CH}_{3}\right), 25.55\left(\mathrm{~s}, \mathrm{CH}_{3}\right), 24.67\left(\mathrm{~s}, \mathrm{CH}_{3}\right), 24.51\left(\mathrm{~s}, \mathrm{CH}_{3}\right), 24.49\left(\mathrm{~s}, \mathrm{CH}_{3}\right), 24.37\left(\mathrm{~s}, \mathrm{CH}_{3}\right)$, and $20.97\left(\mathrm{~s}, \mathrm{CH}_{3}\right) \mathrm{ppm}$.

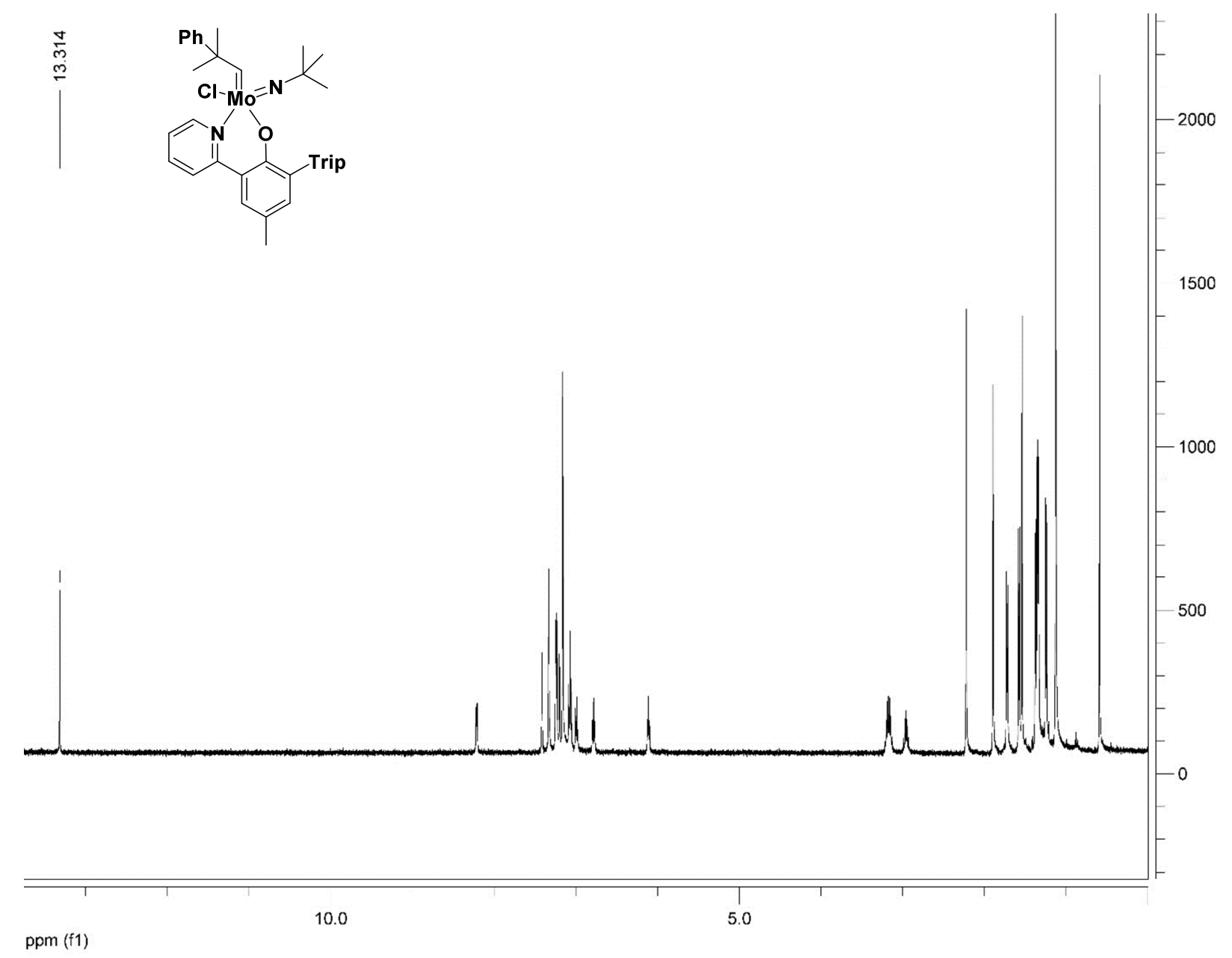

Figure S8. ${ }^{1} \mathrm{H} N M R\left(500 \mathrm{MHz}, \mathrm{C}_{6} \mathrm{D}_{6}\right)$ spectrum and structure of $\mathbf{4}($ TripON).

5(TripON). ${ }^{1} \mathrm{H}$ NMR $\left(500 \mathrm{MHz}, \mathrm{C}_{6} \mathrm{D}_{6}\right) \delta: 12.75\left(\mathrm{~s}, 1 \mathrm{H}, \mathrm{W}=\mathrm{CH}, J_{\mathrm{CH}}=144 \mathrm{~Hz}\right.$, anti), $10.52\left(\mathrm{~s}, 1 \mathrm{H}, \mathrm{W}=\mathrm{CH}, J_{\mathrm{CH}}=\right.$ $114 \mathrm{~Hz}$, syn), 8.12 (dd, $1 \mathrm{H}$, Aromatic- $\mathrm{CH}, \mathrm{J}=6.0,4.7 \mathrm{~Hz}), 7.37(\mathrm{~s}, 1 \mathrm{H}$, Aromatic- $\mathrm{CH}), 7.33(\mathrm{~s}, 1 \mathrm{H}$, Aromatic$\mathrm{CH})$, 7.26-7.21 (m, 2H, Aromatic- $\mathrm{CH}), 7.19(\mathrm{~s}, 1 \mathrm{H}$, Aromatic- $\mathrm{CH}), 6.99-6.96(\mathrm{~m}, 2 \mathrm{H}$, Aromatic- $\mathrm{CH}), 6.95-$ 
$6.91(\mathrm{~m}, 4 \mathrm{H}$, Aromatic- $\mathrm{CH}), 6.58-6.54(\mathrm{~m}, 1 \mathrm{H}$, Aromatic- $\mathrm{CH}), 5.51-5.46(\mathrm{~m}, 1 \mathrm{H}$, Aromatic- $\mathrm{CH}), 3.87$ (br s, $\left.2 \mathrm{H}, \mathrm{CH}\left(\mathrm{CH}_{3}\right)_{2}\right), 3.01$ (sept, $1 \mathrm{H}, \mathrm{CH}\left(\mathrm{CH}_{3}\right)_{2}, J=6.9 \mathrm{~Hz}$ ), 2.75 (sept, $\left.1 \mathrm{H}, \mathrm{CH}\left(\mathrm{CH}_{3}\right)_{2}, J=6.9 \mathrm{~Hz}\right), 2.16\left(\mathrm{~s}, 3 \mathrm{H}, \mathrm{CH}_{3}\right)$, $1.90\left(\mathrm{~s}, 3 \mathrm{H}, \mathrm{CH}_{3}\right), 1.74\left(\mathrm{~s}, 3 \mathrm{H}, \mathrm{CH}_{3}\right), 1.46\left(\mathrm{~d}, 3 \mathrm{H}, \mathrm{CH}\left(\mathrm{CH}_{3}\right)_{2}, J=6.9 \mathrm{~Hz}\right), 1.45\left(\mathrm{~d}, 3 \mathrm{H}, \mathrm{CH}\left(\mathrm{CH}_{3}\right)_{2}, J=6.9 \mathrm{~Hz}\right)$, $1.43\left(\mathrm{~d}, 6 \mathrm{H}, \mathrm{CH}\left(\mathrm{CH}_{3}\right)_{2}, J=6.9 \mathrm{~Hz}\right), 1.28\left(\mathrm{~d}, 6 \mathrm{H}, \mathrm{CH}\left(\mathrm{CH}_{3}\right)_{2}, J=6.9 \mathrm{~Hz}\right), 1.25\left(\mathrm{~d}, 3 \mathrm{H}, \mathrm{CH}\left(\mathrm{CH}_{3}\right)_{2}, J=6.8 \mathrm{~Hz}\right), 1.18$ $\left(\mathrm{d}, 3 \mathrm{H}, \mathrm{CH}\left(\mathrm{CH}_{3}\right)_{2}, J=6.8 \mathrm{~Hz}\right)$, and $0.96\left(\mathrm{br} \mathrm{d}, 6 \mathrm{H}, \mathrm{CH}\left(\mathrm{CH}_{3}\right)_{2}, J=6.4 \mathrm{~Hz}\right) \mathrm{ppm} .{ }^{19} \mathrm{~F} \mathrm{NMR}\left(300 \mathrm{MHz}, \mathrm{C}_{6} \mathrm{D}_{6}\right) \delta$ : -

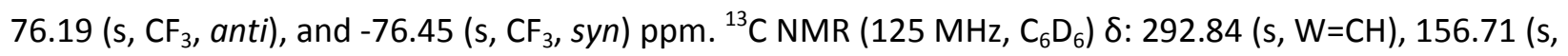
Aromatic-C), 155.31 (s, Aromatic-C), 154.38 (s, Aromatic-CH), 151.62 (s, Aromatic-C), 150.42 (s, Aromatic-C), 148.82 (s, Aromatic-C), 146.62 (s, Aromatic-C), 146.42 (s, Aromatic-C), 146.36 (s, AromaticC), 146.25 (s, Aromatic-C), 138.27 (s, Aromatic-CH), 137.93 (s, Aromatic-CH), 133.93 (s, Aromatic-C), 132.78 (s, Aromatic-C), 130.10 (s, Aromatic-C), 128.51 (s, Aromatic-C), 128.40 (s, Aromatic-C), 127.72 (s, Aromatic-C), 127.63 (s, Aromatic-C), 126.43 (s, Aromatic-CH), 125.81 (s, Aromatic-CH), 125.65 (Aromatic$\mathrm{CH}), 124.69$ (s, Aromatic-CH), 123.20 (s, Aromatic-C), 122.83 (s, Aromatic- $\mathrm{CH}$ ), 121.72 (s, Aromatic- $\mathrm{CH}$ ), 121.53 (s, Aromatic- $\mathrm{CH}$ ), 120.83 (s, Aromatic-CH), 120.57 (s, Aromatic- $\mathrm{CH}$ ), 118.59 (s, $\left.\mathrm{CF}_{3}\right), 48.27$ (s, $\left.\mathrm{C}\left(\mathrm{CH}_{3}\right)_{2} \mathrm{Ph}\right), 35.09$ (s, $\left.\mathrm{CHCH}_{3}\right), 32.16\left(\mathrm{~s}, \mathrm{CH}_{3}\right), 31.65$ (s, $\left.\mathrm{CHCH}_{3}\right), 31.36$ (s, $\left.\mathrm{CHCH}_{3}\right), 29.56\left(\mathrm{~s}, \mathrm{CHCH}_{3}\right), 28.88$ (s, $\mathrm{CH}_{3}$ ), $25.79\left(\mathrm{~s}, \mathrm{CH}_{3}\right), 25.74\left(\mathrm{~s}, \mathrm{CH}_{3}\right), 24.58\left(\mathrm{~s}, \mathrm{CH}_{3}\right), 24.48\left(\mathrm{~s}, \mathrm{CH}_{3}\right), 24.40\left(\mathrm{~s}, \mathrm{CH}_{3}\right), 23.76\left(\mathrm{~s}, \mathrm{CH}_{3}\right), 23.45$ (s, $\left.\mathrm{CH}_{3}\right)$, and $20.82\left(\mathrm{~s}, \mathrm{CH}_{3}\right)$ ppm.

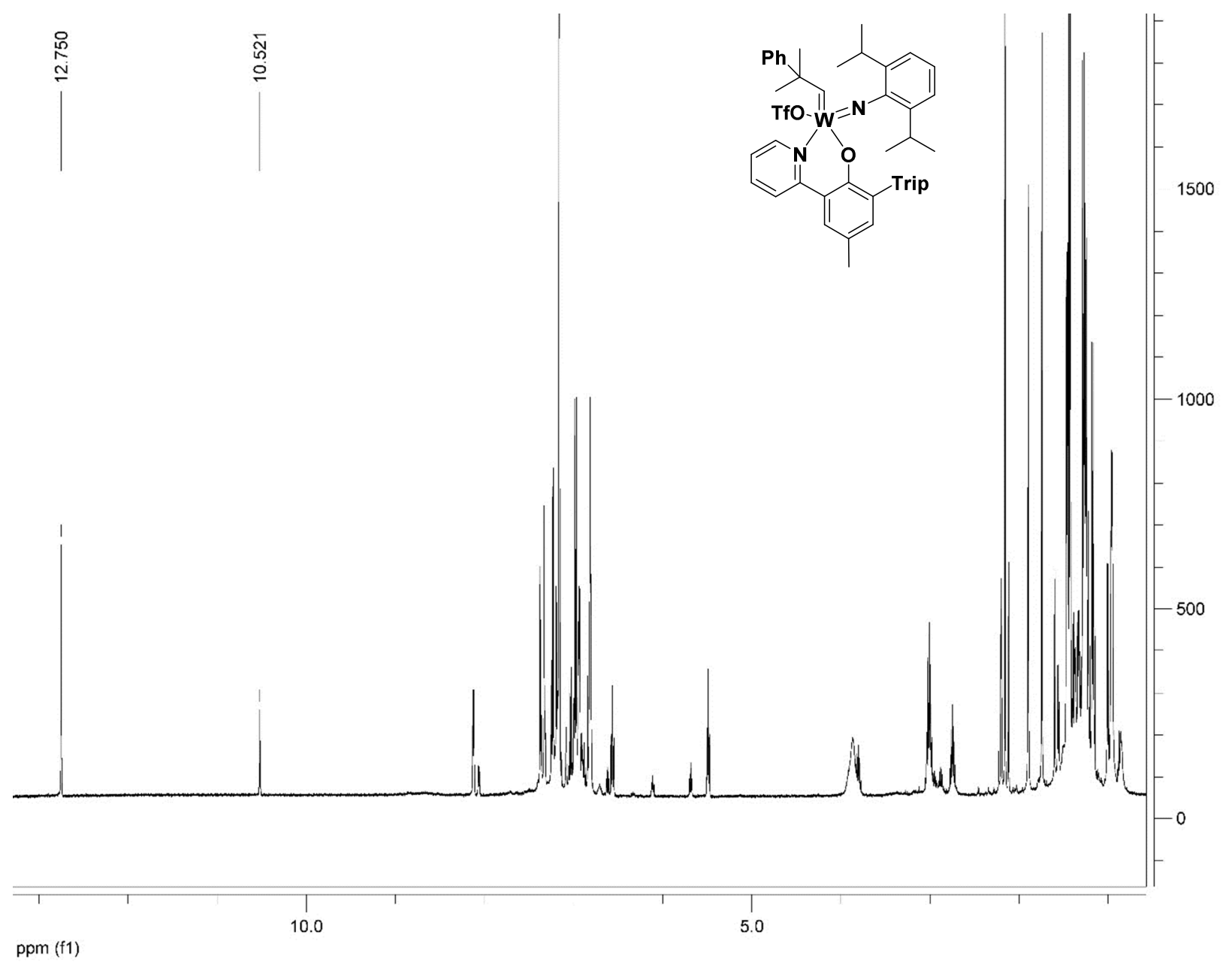

Figure S9. ${ }^{1} \mathrm{H} N M R\left(500 \mathrm{MHz}, \mathrm{C}_{6} \mathrm{D}_{6}\right)$ spectrum and structure of $\mathbf{5}$ (TripON). 
6(TripON). ${ }^{1} \mathrm{H}$ NMR (500 MHz, $\left.\mathrm{C}_{6} \mathrm{D}_{6}\right) \delta: 14.94\left(\mathrm{~s}, 1 \mathrm{H}, \mathrm{Mo}=\mathrm{CH}, J_{\mathrm{CH}}=147 \mathrm{~Hz}\right.$, anti), $13.29\left(\mathrm{~s}, 1 \mathrm{H}, \mathrm{Mo}=\mathrm{CH}, J_{\mathrm{CH}}\right.$ $=119 \mathrm{~Hz}$, syn), $7.84\left(\mathrm{dd}, 1 \mathrm{H}\right.$, Aromatic $\left.-\mathrm{CH}, J_{\mathrm{CH}}=7.0,6.0 \mathrm{~Hz}\right), 7.26-7.21(\mathrm{~m}, 1 \mathrm{H}$, Aromatic- $\mathrm{CH}), 7.20-7.18$ $(\mathrm{m}, 1 \mathrm{H}$, Aromatic- $\mathrm{CH}), 7.15-7.13(\mathrm{~m}, 1 \mathrm{H}$, Aromatic- $\mathrm{CH}), 7.13-7.09(\mathrm{~m}, 1 \mathrm{H}$, Aromatic- $\mathrm{CH}), 6.96-6.86(\mathrm{~m}$, $6 \mathrm{H}$, Aromatic-CH), 6.82-6.75 (m, $1 \mathrm{H}$, Aromatic- $\mathrm{CH}), 6.68$ (ddd, $1 \mathrm{H}$, Aromatic- $\mathrm{CH}, \mathrm{J}=9.1,7.4,1.7 \mathrm{~Hz}), 5.74-$ $5.69\left(\mathrm{~m}, 1 \mathrm{H}\right.$, Aromatic- $\mathrm{CH}$ ), 3.82 (sept, $2 \mathrm{H}, \mathrm{CH}\left(\mathrm{CH}_{3}\right)_{2}, J=6.8 \mathrm{~Hz}$ ), 3.06 (sept, $1 \mathrm{H}, \mathrm{CH}\left(\mathrm{CH}_{3}\right)_{2}, J=6.8 \mathrm{~Hz}$ ), 3.01 (sept, $1 \mathrm{H}, \mathrm{CH}\left(\mathrm{CH}_{3}\right)_{2}, J=6.9 \mathrm{~Hz}$ ), $2.94\left(\right.$ sept, $\left.1 \mathrm{H}, \mathrm{CH}\left(\mathrm{CH}_{3}\right)_{2}, J=6.9 \mathrm{~Hz}\right), 2.23\left(\mathrm{~s}, 3 \mathrm{H}, \mathrm{CH}_{3}\right), 2.14\left(\mathrm{~s}, 3 \mathrm{H}, \mathrm{CH}_{3}\right)$, $1.58\left(\mathrm{~s}, 3 \mathrm{H}, \mathrm{CH}_{3}\right), 1.54\left(\mathrm{~d}, 3 \mathrm{H}, \mathrm{CH}\left(\mathrm{CH}_{3}\right)_{2}, J=6.9 \mathrm{~Hz}\right), 1.43\left(\mathrm{~d}, 6 \mathrm{H}, \mathrm{CH}\left(\mathrm{CH}_{3}\right)_{2}, J=6.9 \mathrm{~Hz}\right), 1.41(\mathrm{~d}, 3 \mathrm{H}$, $\left.\mathrm{CH}\left(\mathrm{CH}_{3}\right)_{2}, J=6.9 \mathrm{~Hz}\right), 1.28\left(\mathrm{~d}, 3 \mathrm{H}, \mathrm{CH}\left(\mathrm{CH}_{3}\right)_{2}, J=6.8 \mathrm{~Hz}\right), 1.24\left(\mathrm{~d}, 6 \mathrm{H}, \mathrm{CH}\left(\mathrm{CH}_{3}\right)_{2}, J=6.8 \mathrm{~Hz}\right), 1.23(\mathrm{~d}, 3 \mathrm{H}$, $\left.\mathrm{CH}\left(\mathrm{CH}_{3}\right)_{2}, J=6.8 \mathrm{~Hz}\right)$, and $1.00\left(\mathrm{~d}, 6 \mathrm{H}, \mathrm{CH}\left(\mathrm{CH}_{3}\right)_{2}, J=6.8 \mathrm{~Hz}\right) \mathrm{ppm} .{ }^{19} \mathrm{~F} \mathrm{NMR}\left(300 \mathrm{MHz}, \mathrm{C}_{6} \mathrm{D}_{6}\right) \delta:-76.30(\mathrm{~s}$,

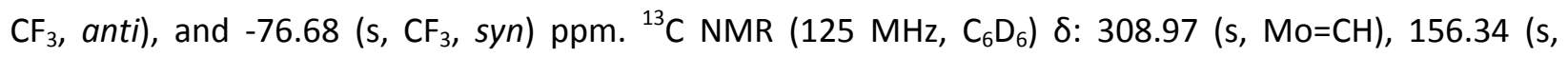
Aromatic-C), 156.32 (s, Aromatic-C), 153.54 (s, Aromatic-CH), 151.11 (s, Aromatic-C), 148.61 (s, Aromatic-C), 147.51 (s, Aromatic-C), 147.13 (s, Aromatic-C), 146.66 (s, Aromatic-C), 146.64 (s, AromaticC), 138.65 (s, Aromatic-CH), 137.49 (s, Aromatic-CH), 133.74 (s, Aromatic-C), 133.08 (s, Aromatic-C), 129.44 (s, Aromatic-CH), 128.52 (s, Aromatic- $\mathrm{CH}$ ), 128.52 (s, Aromatic- $\mathrm{CH}$ ), 126.33 (s, Aromatic-CH), 126.28 (s, Aromatic-CH), 126.01 (s, Aromatic- $\mathrm{CH}$ ), 124.86 (s, Aromaic-C), 122.31 (s, Aromatic-C), 121.28 (s, Aromatic-CH), 121.10 (s, Aromatic-CH), 120.88 (s, Aromatic-CH), 118.91 (s, Aromatic-C), 54.61 (s, $\mathrm{C}\left(\mathrm{CH}_{3}\right)_{2} \mathrm{Ph}$ ), 35.07 (s, $\left.\mathrm{CHCH}_{3}\right), 31.84\left(\mathrm{~s}, \mathrm{CH}_{3}\right), 31.76\left(\mathrm{~s}, \mathrm{CHCH}_{3}\right), 31.63\left(\mathrm{~s}, \mathrm{CHCH}_{3}\right), 30.15\left(\mathrm{~s}, \mathrm{CHCH}_{3}\right), 29.90$ (s, $\left.\mathrm{CH}_{3}\right), 29.68\left(\mathrm{~s}, \mathrm{CH}_{3}\right), 28.91\left(\mathrm{~s}, \mathrm{CH}_{3}\right), 25.48\left(\mathrm{~s}, \mathrm{CH}_{3}\right), 25.07\left(\mathrm{~s}, \mathrm{CH}_{3}\right), 24.51\left(\mathrm{~s}, \mathrm{CH}_{3}\right), 24.43\left(\mathrm{~s}, \mathrm{CH}_{3}\right), 24.05(\mathrm{~s}$, $\left.\mathrm{CH}_{3}\right)$, and $20.78\left(\mathrm{~s}, \mathrm{CH}_{3}\right) \mathrm{ppm}$.

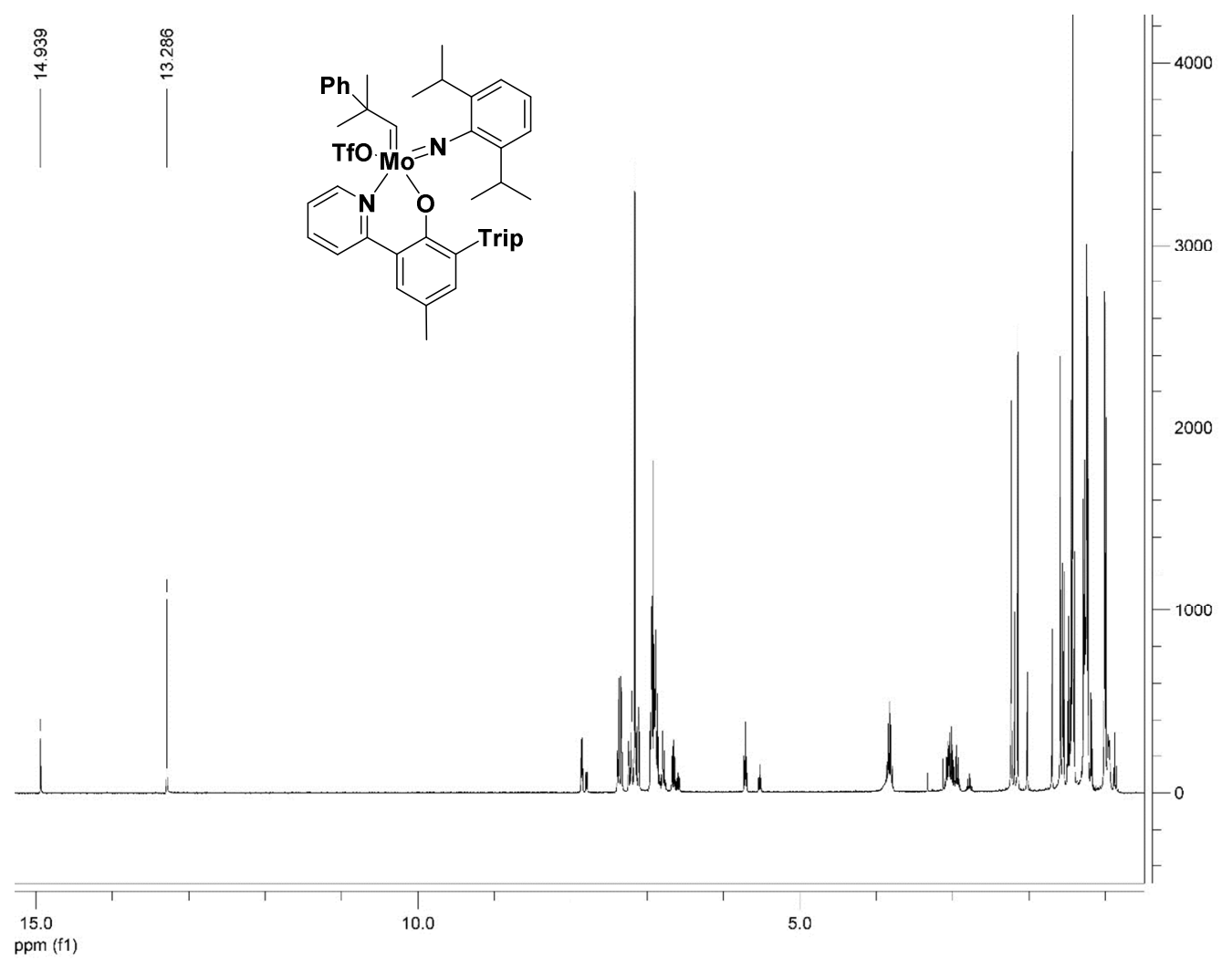

Figure S10. ${ }^{1} \mathrm{H} N \mathrm{NMR}\left(500 \mathrm{MHz}, \mathrm{C}_{6} \mathrm{D}_{6}\right)$ spectrum and structure of $\mathbf{6}($ TripON). 
7(TripON). ${ }^{1} \mathrm{H}$ NMR (500 MHz, $\left.\mathrm{C}_{6} \mathrm{D}_{6}\right) \delta: 8.96(\mathrm{~d}, 1 \mathrm{H}$, Aromatic- $\mathrm{CH}, J=5.7 \mathrm{~Hz}$, minor/adduct), $8.42(\mathrm{~d}, 1 \mathrm{H}$, Aromatic- $\mathrm{CH}, J=5.7 \mathrm{~Hz}$, major/adduct-free), $7.45(\mathrm{~d}, 1 \mathrm{H}$, Aromatic- $\mathrm{CH}, J=8.1 \mathrm{~Hz}$, minor/adduct), 7.36 (d, $1 \mathrm{H}$, Aromatic- $\mathrm{CH}, \mathrm{J}=8.2 \mathrm{~Hz}$, major/adduct-free $), 7.34-7.30(\mathrm{~m}, 2 \mathrm{H}$, Aromatic- $\mathrm{CH}$, major/adduct-free $)$, $7.28(\mathrm{~s}, 1 \mathrm{H}$, Aromatic- $\mathrm{CH}$, minor/adduct), 7.23 (s, $1 \mathrm{H}$, Aromatic- $\mathrm{CH}$, minor/adduct), 7.20-7.16 (m, 3H, Aromatic- $\mathrm{CH}$, minor/adduct), 7.10 (s, Aromatic- $\mathrm{CH}$, major/adduct-free), 6.99 (s, $1 \mathrm{H}$, Aromatic- $\mathrm{CH}$, minor/adduct), $6.98(\mathrm{~s}, 1 \mathrm{H}$, Aromatic- $\mathrm{CH}$, minor/adduct), 6.94-6.89 (m, 1H, Aromatic- $\mathrm{CH}$, major/adductfree, and $\mathrm{m}, 1 \mathrm{H}$, Aromatic- $\mathrm{CH}$, minor/adduct), $6.87(\mathrm{~s}, 3 \mathrm{H}$, Aromatic- $\mathrm{CH}$, major/adduct-free), $6.76(\mathrm{t}, 1 \mathrm{H}$, Aromatic- $\mathrm{CH}, J=7.7 \mathrm{~Hz}$, major/adduct-free), $6.36(\mathrm{t}, 1 \mathrm{H}$, Aromatic- $\mathrm{CH}, J=6.4 \mathrm{~Hz}), 5.89(\mathrm{t}, 1 \mathrm{H}$, Aromatic$\mathrm{CH}, J=6.6 \mathrm{~Hz}$, major/adduct-free $), 3.97-3.85\left(\mathrm{~m}, 2 \mathrm{H}, \mathrm{OCH}_{2}\right.$, minor/adduct, $\mathrm{m}, 2 \mathrm{H}, \mathrm{CH}\left(\mathrm{CH}_{3}\right)_{2}$, minor/adduct, and $\mathrm{m}, 1 \mathrm{H}, \mathrm{CH}_{2}=\mathrm{CH}_{2}$, major/adduct-free), 3.67-3.58 (dq, $2 \mathrm{H}, \mathrm{OCH}_{2}, J=12.9,6.9 \mathrm{~Hz}$, minor/adduct $), \quad 3.47-3.34\left(\mathrm{~m}, 3 \mathrm{H}, \mathrm{CH}\left(\mathrm{CH}_{3}\right)_{2}\right.$, major/adduct-free $), 3.20-3.13\left(\mathrm{~m}, 1 \mathrm{H}, \mathrm{CH}_{2}=\mathrm{CH}_{2}\right.$, minor/adduct), 3.01 (sept, $1 \mathrm{H}, \mathrm{CH}\left(\mathrm{CH}_{3}\right)_{2}$, major/adduct-free), 2.92-2.72 (m, $3 \mathrm{H}, \mathrm{CH}\left(\mathrm{CH}_{3}\right)_{2}$, minor/adduct, $\mathrm{m}, 1 \mathrm{H}, \mathrm{CH}_{2}=\mathrm{CH}_{2}$, minor/adduct, and $\mathrm{m}, 1 \mathrm{H}, \mathrm{CH}_{2}=\mathrm{CH}_{2}$, major/adduct-free $), 2.69-2.58\left(\mathrm{~m}, 1 \mathrm{H}, \mathrm{CH}\left(\mathrm{CH}_{3}\right)_{2}\right.$, major/adduct-free $), 2.56-2.49\left(\mathrm{~m}, 1 \mathrm{H}, \mathrm{CH}_{2}=\mathrm{CH}_{2}\right.$, minor/adduct $), 2.49-2.36\left(\mathrm{~m}, 1 \mathrm{H}, \mathrm{CH}_{2}=\mathrm{CH}_{2}\right.$, minor/adduct, and $\mathrm{m}, 1 \mathrm{H}, \mathrm{CH}_{2}=\mathrm{CH}_{2}$, major/adduct-free), $2.26\left(\mathrm{~s}, 3 \mathrm{H}, \mathrm{CH}_{3}\right.$, minor/adduct), $2.13\left(\mathrm{~s}, 3 \mathrm{H}, \mathrm{CH}_{3}\right.$, major/adduct-free), 1.92-1.80 (m, $1 \mathrm{H}, \mathrm{CH}_{2}=\mathrm{CH}_{2}$, major/adduct-free), $1.57\left(\mathrm{~d}, 3 \mathrm{H}, \mathrm{CH}\left(\mathrm{CH}_{3}\right)_{2}, J=6.7 \mathrm{~Hz}\right.$, minor/adduct), 1.48 (d, $3 \mathrm{H}, \mathrm{CH}\left(\mathrm{CH}_{3}\right)_{2}, J=6.8 \mathrm{~Hz}$, minor/adduct $), 1.47-1.40\left(\mathrm{~m}, 9 \mathrm{H}, \mathrm{CH}\left(\mathrm{CH}_{3}\right)_{2}\right.$, major/adduct-free), $1.34\left(\mathrm{~d}, 6 \mathrm{H}, \mathrm{CH}\left(\mathrm{CH}_{3}\right)_{2}, \mathrm{~J}=6.7 \mathrm{~Hz}\right.$, minor/adduct $), 1.32-1.19\left(\mathrm{~m}, 9 \mathrm{H}, \mathrm{CH}\left(\mathrm{CH}_{3}\right)_{2}\right.$, minor/adduct, and $\mathrm{m}, 15 \mathrm{H}, \mathrm{CH}\left(\mathrm{CH}_{3}\right)_{2}$, major/adduct-free), $1.17\left(\mathrm{~d}, 6 \mathrm{H}, \mathrm{CH}\left(\mathrm{CH}_{3}\right)_{2}, J=6.8 \mathrm{~Hz}\right.$, minor/adduct), 1.08 (d, $3 \mathrm{H}, \mathrm{CH}\left(\mathrm{CH}_{3}\right)_{2}, J=6.8 \mathrm{~Hz}$, minor/adduct), 1.04-0.93 (m, $6 \mathrm{H}, \mathrm{CH}\left(\mathrm{CH}_{3}\right)_{2}$, major/adduct-free), and $0.82\left(\mathrm{t}, 6 \mathrm{H}, \mathrm{CH}_{2} \mathrm{CH}_{3}, J=7.0 \mathrm{~Hz}\right.$, minor/adduct) ppm. ${ }^{19} \mathrm{~F} \mathrm{NMR}\left(300 \mathrm{MHz}, \mathrm{C}_{6} \mathrm{D}_{6}\right) \delta$ : 77.23 (major/adduct-free), and -77.70 (minor/adduct) ppm. ${ }^{13} \mathrm{C} \mathrm{NMR} \mathrm{(125} \mathrm{MHz,} \mathrm{C}_{6} \mathrm{D}_{6}$ ) $\delta: 158.31$ (s, Aromatic-C), 157.47 (s, Aromatic-C), 157.36 (s, Aromatic-C), 154.40 (s, Aromatic-C), 152.73 (s, Aromatic$\mathrm{CH}$ ), 152.67 (s, Aromatic-CH), 152.05 (s, Aromatic-C), 151.42 (s, Aromatic-C), 149.81 (s, Aromatic-C), 149.36 (s, Aromatic-C), 148.74 (s, Aromatic-C), 147.90 (s, Aromatic-C), 147.55 (br s, Aromatic-C), 146.82 (s, Aromatic-C), 146.58 (br s, Aromatic-C), 146.12 (br s, Aromatic-C), 138.29 (s, Aromatic-CH), 138.21 (s, Aromatic-CH), 136.96 (s, Aromatic-CH), 136.93 (s, Aromatic-CH), 135.91 (s, Aromatic-C), 133.21 (s, Aromatic-C), 132.87 (s, Aromatic-C), 130.45 (s, Aromatic-C), 129.92 (s, Aromatic-C), 129.79 (s, Aromatic$\mathrm{CH}), 129.19$ (s, Aromatic-CH), 127.53 (br s, Aromatic-CH), 126.66 (s, Aromatic-CH), 126.23 (s, AromaticC), 125.63 (s, Aromatic-C), 125.04 (s, Aromatic-CH), 124.65 (s, Aromatic-CH), 123.49 (s, Aromatic- $\mathrm{CH}$ ), 121.56 (s, Aromatic-CH), 121.41 (s, Aromatic-CH), 121.21 (br s, Aromatic-CH), 121.08-120.71 (s, Aromatic-CH), 120.05 (s, Aromatic- $\mathrm{CH}$ ), 129.4 (br s, $\mathrm{CF}_{3}$ ), 118.78 (br s, $\left.\mathrm{CF}_{3}\right), 69.65$ (s, $\mathrm{OCH}_{2}$, minor/adduct), 63.90 (s, $\mathrm{CH}_{2}=\mathrm{CH}_{2}$, minor/adduct), 63.44 ( $\mathrm{s}, \mathrm{CH}_{2}=\mathrm{CH}_{2}$, minor/adduct), 61.89 (br s, $\mathrm{CH}_{2}=\mathrm{CH}_{2}$, major/adduct-free), 59.92 (br s, $\mathrm{CH}_{2}=\mathrm{CH}_{2}$, major/adduct-free), 35.06 (s, $\mathrm{CHCH}_{3}$, major/adductfree), 34.91 (s, $\mathrm{CHCH}_{3}$, minor/adduct), 31.56 (s, $\mathrm{CHCH}_{3}$, minor/adduct), 31.40 (s, $\mathrm{CHCH}_{3}$, major/adductfree), 30.84 (s, $\mathrm{CHCH}_{3}$, minor/adduct), 30.47 (s, $\mathrm{CHCH}_{3}$, major/adduct-free), 30.46 (s, $\mathrm{CHCH}_{3}$, major/adduct-free), 27.47 (s, $\mathrm{CHCH}_{3}$, minor/adduct), $26.25\left(\mathrm{~s}, \mathrm{CHCH}_{3}\right), 25.22\left(\mathrm{~s}, \mathrm{CHCH}_{3}\right), 25.10\left(\mathrm{~s}, \mathrm{CHCH}_{3}\right)$, 24.89 (br s, $\left.\mathrm{CHCH}_{3}\right), 24.71-24.32$ (m, (s, $\left.\mathrm{CHCH}_{3}\right), 24.05$ (br s, $\left.\mathrm{CHCH}_{3}\right)$, 23.93-23.54 (m, $\left.\mathrm{CHCH}_{3}\right), 20.91$ (s, $\mathrm{CHCH}_{3}$, minor/adduct), 20.86 (s, $\mathrm{CHCH}_{3}$, major/adduct-free), and 14.31 (s, $\mathrm{CH}_{2} \mathrm{CH}_{3}$, minor/adduct) ppm. It should be noted that free ether was seen in the ${ }^{13} \mathrm{C}$ NMR spectrum. 


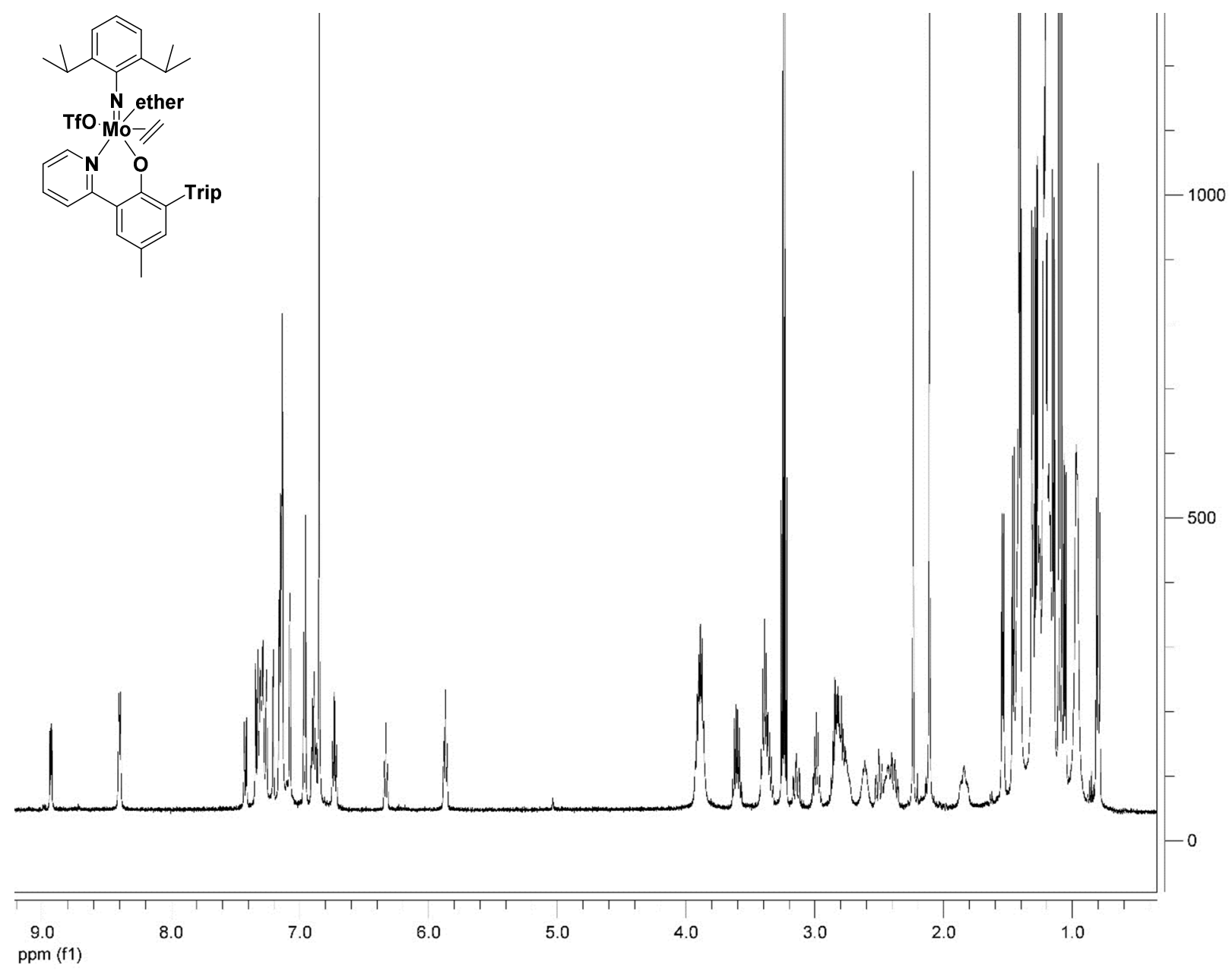

Figure S11. ${ }^{1} \mathrm{H} N M R\left(500 \mathrm{MHz}, \mathrm{C}_{6} \mathrm{D}_{6}\right)$ spectrum and structure of 7(TripON). 


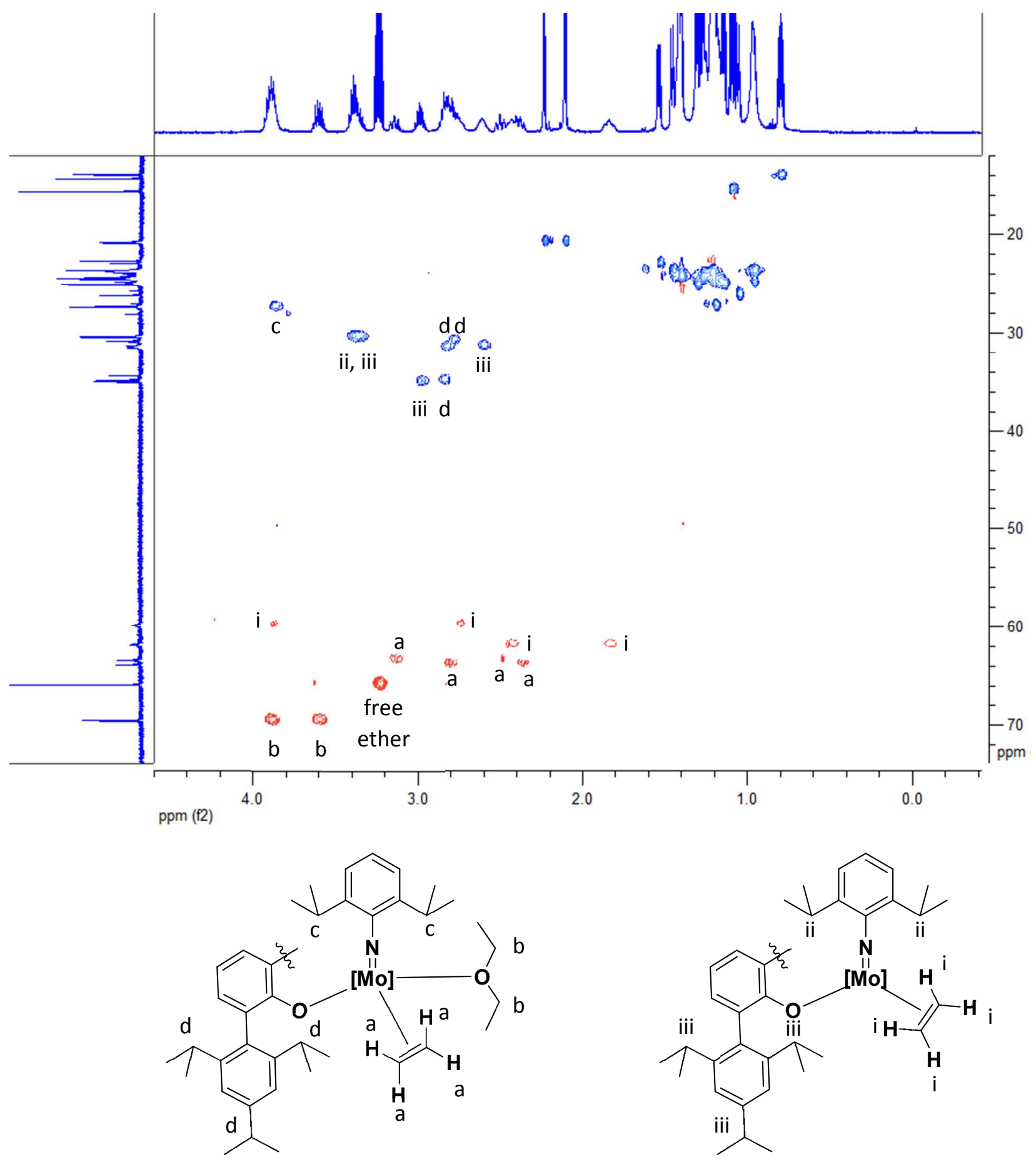

Figure S12. gHSQC NMR (500 MHz, $\left.\mathrm{C}_{6} \mathrm{D}_{6}\right)$ spectrum and partial structures of 7 (TripON) in solution, as well as numbering of relevant resonances. 


\section{CATALYTIC STUDIES}

General Considerations. All procedures and manipulations were performed under an argon or nitrogen atmosphere using standard Schlenk-line and glovebox techniques unless stated otherwise. Reagents were purchased from commercial sources and used without further purification unless stated otherwise. All glassware was oven-dried or flame-dried prior to use unless stated otherwise. Octane, 1-hexene, and 1-octene were distilled over $\mathrm{CaH}_{2}$ under a nitrogen atmosphere, stirred over NaK for several hours, filtered through Celite and stored over molecular sieves in a nitrogen-filled glovebox prior to use. Deuterated solvents were purchased from Cambridge Isotope Laboratories, degassed, and dried over activated molecular sieves prior to use.

1-Hexene Homocoupling. A solution of 100 eq. 1-hexene in $0.5 \mathrm{~mL}$ solvent was added to $0.010 \mathrm{~g}$ of catalyst ( 1 eq.) and loaded into a J-young tube. The reaction was sealed and immersed into a preheated oil bath at various temperatures. Progress of the reaction was followed by $1 \mathrm{H}$ NMR spectroscopy and after $24 \mathrm{~h}$ the reaction mixture was cooled, run through an alumina plug, diluted with pentane, and confirmed by GC with a mesitylene internal standard.

Table S1. Homocoupling of 1-hexene by 1-6.

\begin{tabular}{|c|c|c|c|c|c|c|c|}
\hline Cat. $^{a}$ & Solvent & $\begin{array}{c}\text { Temp. } \\
\left({ }^{\circ} \mathrm{C}\right)\end{array}$ & $\begin{array}{c}\text { Time } \\
\text { (h) }\end{array}$ & $\begin{array}{c}\text { trans-2- } \\
\text { hexene (\%) }\end{array}$ & $\begin{array}{c}\text { cis-2-hexene } \\
\text { (\%) }\end{array}$ & $\begin{array}{c}\text { 5-decene } \\
(\%)\end{array}$ & $\begin{array}{c}\text { E/Z Ratio } \\
\text { (\%:\%) }\end{array}$ \\
\hline 1(MesON) & $\mathrm{C}_{6} \mathrm{D}_{6}$ & $\mathrm{rt}$ & 24 & $<1$ & $<1$ & $<1$ & $\mathrm{~N} / \mathrm{A}$ \\
\hline 1(MesON) & $\mathrm{C}_{6} \mathrm{D}_{6}$ & 50 & 24 & $<1$ & $<1$ & 1.9 & $75 / 25$ \\
\hline 1 (MesON) & $\mathrm{C}_{6} \mathrm{D}_{6}$ & 80 & 24 & $<1$ & $<1$ & 6.0 & $56 / 44$ \\
\hline 1(MesON) & $\mathrm{CDCl}_{3}$ & $\mathrm{rt}$ & 24 & $<1$ & $<1$ & 5.9 & $53 / 47$ \\
\hline 2(MesON) & $\mathrm{CDCl}_{3}$ & $\mathrm{rt}$ & 24 & $<1$ & $<1$ & $<1$ & N/A \\
\hline 3(MesON) & $\mathrm{CDCl}_{3}$ & $\mathrm{rt}$ & 24 & $<1$ & $<1$ & 8.1 & $55 / 45$ \\
\hline 4(MesON) & $\mathrm{CDCl}_{3}$ & $\mathrm{rt}$ & 24 & $<1$ & $<1$ & $<1$ & $\mathrm{~N} / \mathrm{A}$ \\
\hline 1(MesON) & $\mathrm{CDCl}_{3}$ & 50 & 24 & $<1$ & $<1$ & 12.0 & $54 / 46$ \\
\hline 1(MesON) & $\mathrm{CDCl}_{3}$ & 80 & 24 & $<1$ & $<1$ & 15.1 & $63 / 37$ \\
\hline 2(MesON) & $\mathrm{CDCl}_{3}$ & 80 & 24 & $<1$ & $<1$ & $<1$ & $\mathrm{~N} / \mathrm{A}$ \\
\hline 3(MesON) & $\mathrm{CDCl}_{3}$ & 80 & 24 & $<1$ & $<1$ & 12.0 & $58 / 42$ \\
\hline 4(MesON) & $\mathrm{CDCl}_{3}$ & 80 & 24 & $<1$ & $<1$ & 2.5 & $67 / 33$ \\
\hline 1(MesON) & $C_{7} D_{8}$ & 120 & 24 & 22.4 & 13.4 & 2.7 & $49 / 51$ \\
\hline 1(TripON) & $C_{6} D_{6}$ & $\mathrm{rt}$ & 24 & $<1$ & $<1$ & $<1$ & $\mathrm{~N} / \mathrm{A}$ \\
\hline 1(TripON) & $\mathrm{C}_{6} \mathrm{D}_{6}$ & 50 & 24 & $<1$ & $<1$ & 1.2 & $88 / 12$ \\
\hline 1(TripON) & $\mathrm{C}_{6} \mathrm{D}_{6}$ & 80 & 24 & $<1$ & $<1$ & 12.1 & $46 / 54$ \\
\hline 1(TripON) & $\mathrm{CDCl}_{3}$ & $\mathrm{rt}$ & 24 & $<1$ & $<1$ & 4.7 & $62 / 38$ \\
\hline 2(TripON) & $\mathrm{CDCl}_{3}$ & $\mathrm{rt}$ & 24 & $<1$ & $<1$ & $<1$ & $\mathrm{~N} / \mathrm{A}$ \\
\hline 3(TripON) & $\mathrm{CDCl}_{3}$ & $\mathrm{rt}$ & 24 & $<1$ & $<1$ & 7.9 & $57 / 43$ \\
\hline 4(TripON) & $\mathrm{CDCl}_{3}$ & $\mathrm{rt}$ & 24 & $<1$ & $<1$ & $<1$ & $N / A$ \\
\hline 5(TripON) & $\mathrm{CDCl}_{3}$ & $\mathrm{rt}$ & 24 & $<1$ & $<1$ & 6.5 & $58 / 42$ \\
\hline 6(TripON) & $\mathrm{CDCl}_{3}$ & $\mathrm{rt}$ & 24 & $<1$ & $<1$ & $<1$ & $\mathrm{~N} / \mathrm{A}$ \\
\hline
\end{tabular}




\begin{tabular}{l|c|c|c|c|c|c|c}
$\mathbf{1}$ (TripON) & $\mathrm{CDCl}_{3}$ & 50 & 24 & $<1$ & $<1$ & 18.7 & $66 / 34$ \\
$\mathbf{1}$ (TripON) & $\mathrm{CDCl}_{3}$ & 80 & 24 & $<1$ & $<1$ & 31.8 & $69 / 31$ \\
$\mathbf{2}$ (TripON) & $\mathrm{CDCl}_{3}$ & 80 & 24 & $<1$ & $<1$ & $<1$ & N/A \\
$\mathbf{3 ( T r i p O N )}$ & $\mathrm{CDCl}_{3}$ & 80 & 24 & $<1$ & $<1$ & 10.1 & $65 / 35$ \\
$\mathbf{4 ( T r i p O N )}$ & $\mathrm{CDCl}_{3}$ & 80 & 24 & $<1$ & $<1$ & $<1$ & N/A \\
$\mathbf{5 ( T r i p O N )}$ & $\mathrm{CDCl}_{3}$ & 80 & 24 & $<1$ & $<1$ & 11.9 & $52 / 48$ \\
$\mathbf{6 ( T r i p O N )}$ & $\mathrm{CDCl}_{3}$ & 80 & 24 & $<1$ & $<1$ & 7.3 & $56 / 44$ \\
$\mathbf{1}$ (TripON) & $\mathrm{C}_{7} \mathrm{D}_{8}$ & 120 & 24 & 2.0 & 1.2 & 17.8 & $41 / 59$
\end{tabular}

${ }^{a} 1 \mathrm{~mol} \%$ catalyst in $0.5 \mathrm{~mL}$ solvent, in a sealed J-Young tube, conversions and E/Z ratios determined by GC.

1-Octene Homocoupling. A solution of 100 eq. 1-octene in $0.5 \mathrm{~mL}$ solvent was added to $0.010 \mathrm{~g}$ of catalyst (1 eq.) and loaded into a J-young tube. The reaction was exposed to an N2 atmosphere and immersed into a preheated oil bath at $120^{\circ} \mathrm{C}$. After $24 \mathrm{~h}$ the reaction mixture was cooled, run through an alumina plug, diluted with pentane, and quantified by GC with a mesitylene internal standard.

Table S2. Homocoupling of 1-octene by 1 (MesON) and 1(TrippON).

\begin{tabular}{l|l|l|l|l|l|l|l}
\hline \multicolumn{1}{c|}{ Cat. $^{\boldsymbol{a}}$} & Solvent & $\begin{array}{c}\text { Temp. } \\
\left.\mathbf{(}^{\circ} \mathbf{C}\right)\end{array}$ & $\begin{array}{c}\text { Time } \\
\text { (h) }\end{array}$ & $\begin{array}{c}\text { trans-2- } \\
\text { octene (\%) }\end{array}$ & $\begin{array}{c}\text { cis-2- } \\
\text { octene (\%) }\end{array}$ & $\begin{array}{c}\text { 7-tetradecene } \\
\text { (\%) }\end{array}$ & $\begin{array}{c}\text { E/Z Ratio } \\
\text { (\%:\%) }\end{array}$ \\
\hline $\mathbf{1}$ (MesON) & $\mathrm{C}_{7} \mathrm{D}_{8}$ & 120 & 24 & 2.2 & 1.6 & 9.8 & $61 / 39$ \\
$\mathbf{1}$ (MesON) & $\mathrm{C}_{7} \mathrm{H}_{5} \mathrm{~F}_{3}$ & 120 & 24 & 2.8 & 2.1 & 11.7 & $59 / 41$ \\
$\mathbf{1}$ (MesON) & $\mathrm{C}_{6} \mathrm{H}_{5} \mathrm{Cl}$ & 120 & 24 & 2.0 & 1.7 & 10.0 & $54 / 45$ \\
$\mathbf{1}($ TripON) & $\mathrm{C}_{7} \mathrm{D}_{8}$ & 120 & 24 & $<1$ & $<1$ & 52.9 & $51 / 49$ \\
$\mathbf{1}$ (TripON) & $\mathrm{C}_{7} \mathrm{H}_{5} \mathrm{~F}_{3}$ & 120 & 24 & $<1$ & $<1$ & 55.1 & $59 / 41$ \\
$\mathbf{1}($ TripON) & $\mathrm{C}_{6} \mathrm{H}_{5} \mathrm{Cl}$ & 120 & 24 & $<1$ & $<1$ & 38.6 & $55 / 45$
\end{tabular}

${ }^{a} 1 \mathrm{~mol} \%$ catalyst in $0.5 \mathrm{~mL}$ solvent, in a J-Young tube under an $\mathrm{N}_{2}$ atmosphere, conversions and $\mathrm{E} / \mathrm{Z}$ ratios determined by GC.

General Procedure for AM. A stock solution of $30.7 \mathrm{mg} \operatorname{Ir}(t$-BuPOCOP $)\left(\mathrm{C}_{2} \mathrm{H}_{4}\right)$ in $3.5 \mathrm{~g} n$-octane was prepared. In a J-Young tube, $0.5 \mathrm{~mL}$ of the stock solution and $1[\mathrm{MesON}]$ or 1 [TripON] (1 eq.) were combined. The reaction was sealed and immersed into a preheated oil bath at 150 or $200{ }^{\circ} \mathrm{C}$. After 4 days the reaction mixture was cooled, run through an alumina plug. The filtrate was diluted with pentane, and AM was quantified by GC with a mesitylene internal standard.

Table S3. AM of $n$-octene by $\operatorname{Ir}($ BuPOCOP $)\left(\mathrm{C}_{2} \mathrm{H}_{4}\right)$ and 1-6.

\begin{tabular}{|c|c|c|c|c|c|c|c|c|c|c|}
\hline Cat. $^{a}$ & $\begin{array}{c}\text { Temp. } \\
\left({ }^{\circ} \mathrm{C}\right)\end{array}$ & $\begin{array}{c}\text { Time } \\
\text { (h) }\end{array}$ & $\begin{array}{c}{\left[\mathrm{C}_{7}\right]} \\
(\mathrm{mM})\end{array}$ & $\begin{array}{c}{\left[\mathrm{C}_{9}\right]} \\
(\mathrm{mM})\end{array}$ & $\begin{array}{c}{\left[\mathrm{C}_{10}\right]} \\
(\mathrm{mM})\end{array}$ & $\begin{array}{c}{\left[\mathrm{C}_{11}\right]} \\
(\mathrm{mM})\end{array}$ & $\begin{array}{c}{\left[\mathrm{C}_{12}\right]} \\
(\mathrm{mM})\end{array}$ & $\begin{array}{c}{\left[\mathrm{C}_{13}\right]} \\
(\mathrm{mM})\end{array}$ & $\begin{array}{c}{\left[\mathrm{C}_{14}\right]} \\
(\mathrm{mM})\end{array}$ & $\begin{array}{l}\text { Total } \\
\text { (mM) }\end{array}$ \\
\hline 1(MesON) & 150 & 96 & 1.35 & 1.34 & 1.28 & 1.09 & 0.49 & 0.30 & 0.17 & 5.72 \\
\hline 1(MesON) & 200 & 96 & 4.92 & 4.45 & 3.47 & 2.06 & 1.43 & 0.64 & 0.24 & 17.21 \\
\hline 1(TripON) & 150 & 96 & 7.39 & 7.21 & 5.84 & 3.55 & 2.41 & 1.13 & 0.25 & 27.78 \\
\hline 1(TripON) & 200 & 96 & 18.77 & 18.58 & 13.15 & 9.64 & 6.75 & 2.57 & 0.77 & 70.23 \\
\hline 2(MesON) & 150 & 96 & 0 & 0 & 0 & 0 & 0 & 0 & 0 & 0 \\
\hline
\end{tabular}




\begin{tabular}{l|l|l|l|l|l|l|l|l|l|l}
$\mathbf{2}$ (TripON) & 150 & 96 & 0 & 0 & 0 & 0 & 0 & 0 & 0 & 0 \\
3(MesON) & 150 & 96 & 0 & 0 & 0 & 0 & 0 & 0 & 0 & 0 \\
3(TripON) & 150 & 96 & 0 & 0 & 0 & 0 & 0 & 0 & 0 & 0 \\
4(MesON) & 150 & 96 & 0 & 0 & 0 & 0 & 0 & 0 & 0 & 0 \\
4(TripON) & 150 & 96 & 7.16 & 5.88 & 5.18 & 2.46 & 2.16 & 0.93 & 0.26 & 48.06 \\
5(TripON) & 150 & 96 & 10.85 & 10.84 & 9.65 & 6.24 & 4.97 & 1.75 & 0.35 & 89.29 \\
6(TripON) & 150 & 96 & 4.27 & 3.78 & 2.57 & 2.16 & 1.88 & 1.58 & 0.93 & 34.03
\end{tabular}

${ }^{a} 6.4 \mathrm{mM} \mathrm{W}$ catalyst, $6.5 \mathrm{mM}$ Ir catalyst in $0.5 \mathrm{~mL}$ octane in a sealed J-Young tube, conversions determined by GC with mesitylene as an internal standard.

General Procedure for 1-Octene Isomerization/Coupling. A J-Young tube was charged with a suspension of $0.005 \mathrm{~g} 7\left[\right.$ TripON] in $0.5 \mathrm{~mL} 1$-octene. The system was sealed and heated to 100 or $150{ }^{\circ} \mathrm{C}$ for four days. The reaction mixture was filtered through basic alumina and placed under a hydrogen atmosphere with $\sim 0.005 \mathrm{~g} 5 \% \mathrm{Pd} / \mathrm{C}$. The solution was stirred overnight and filtered through Celite. The conversion was quantified by GC with a mesitylene internal standard.

Table S4. Isomerization/Coupling of 1-octene by 7(TripON).

\begin{tabular}{|c|c|c|c|c|c|c|c|c|c|c|}
\hline Cat. $^{a}$ & $\begin{array}{c}\text { Temp. } \\
\left({ }^{\circ} \mathrm{C}\right)\end{array}$ & $\begin{array}{c}\text { Time } \\
\text { (h) }\end{array}$ & $\begin{array}{l}{\left[\mathrm{C}_{7}\right]} \\
(\mathrm{M})\end{array}$ & $\begin{array}{l}{\left[\mathrm{C}_{9}\right]} \\
\text { (M) }\end{array}$ & $\begin{array}{c}{\left[\mathrm{C}_{10}\right]} \\
(\mathrm{M})\end{array}$ & $\begin{array}{l}{\left[\mathrm{C}_{11}\right]} \\
(\mathrm{M})\end{array}$ & $\begin{array}{c}{\left[\mathrm{C}_{12}\right]} \\
(\mathrm{M})\end{array}$ & $\begin{array}{c}{\left[\mathrm{C}_{13}\right]} \\
(\mathrm{M})\end{array}$ & $\begin{array}{c}{\left[\mathrm{C}_{14}\right]} \\
(\mathrm{M})\end{array}$ & $\begin{array}{c}\text { Total } \\
\text { (M) }\end{array}$ \\
\hline 1(MesON) & 100 & 96 & $<1$ & $<1$ & $<1$ & $<1$ & $<1$ & $<1$ & $<1$ & $<1$ \\
\hline 1 (MesON) & 150 & 96 & 55.4 & 95.6 & 15.7 & 14.9 & 67.1 & 76.7 & 89.3 & 414.7 \\
\hline
\end{tabular}

${ }^{a} 11 \mathrm{mM}$ Mo catalyst in $0.5 \mathrm{~mL}$ 1-octene in a sealed J-Young tube, conversions determined by GC with mesitylene as an internal standard.

\section{X-RAY DIFFRACTION STUDIES}

General Considerations. Low-temperature diffraction data were collected on a Bruker-AXS X8 Kappa Duo diffractometer coupled to a SMART Apex2 CCD detector with Mo $K_{\alpha}$ radiation $(\lambda=0.71073 \AA$ ) from an $I \mu S$ micro-source, performing $\phi$-and $\omega$-scans. The structures were solved by direct methods using SHELXT ${ }^{5}$ and refined against $F^{2}$ on all data by full-matrix least squares with SHELXL-2015 ${ }^{6}$ following established refinement strategies. ${ }^{7}$ All non-hydrogen atoms were refined anisotropically. Except when noted otherwise below, all hydrogen atoms were included into the model at geometrically calculated positions and refined using a riding model. The isotropic displacement parameters of all hydrogen atoms were fixed to 1.2 times the $U$ value of the atoms they are linked to (1.5 times for methyl groups). Coordinates for hydrogen atoms on the carbon binding directly to the metal were taken from the difference Fourier Synthesis. Those hydrogen atoms were subsequently refined semi-freely with the help of distance restraints (target $\mathrm{C}-\mathrm{H}$ distance $0.95(2) \AA$ ), while constraining their $U_{\text {iso }}$ values as described above. All disorders mentioned below were refined with the help of similarity restraints on 12 and 1-3 distances and displacement parameters as well as rigid bond restraints for anisotropic displacement parameters. Details of the data quality and a summary of the residual values of the refinements are listed in tables below. 
Compound x8_16005 (2(MesON)) crystallizes in the monoclinic space group $P 2_{1} / n$ with two molecules of x8_16005 and two molecules of toluene in the asymmetric unit. The alkylidene group of one of the independent molecules was refined as disordered over two positions using the abovementioned restraints. The crystal was a two-component non-merohedral twin. Two independent orientation matrices for the unit cell were found using the program CELL_NOW, ${ }^{8}$ and data reduction taking into account the twinning was performed with SAINT ${ }^{9}$ The program TWINABS $^{10}$ was used to perform absorption correction and to set up the HKLF4 format file for structure refinement. Refinement against an HKLF5 format file was also attempted, however the HKLF4 format file yielded a better model.

Compound x8_16023 (3(TripON)) crystallizes in the monoclinic space group C2/c with one molecule of x8_ 16023 and one pentane molecule per asymmetric unit. The pentane molecule was refined as disordered over two independent positions using the abovementioned restraints.

Compound $\mathbf{x 8} \mathbf{8 6 0 6 4}(\mathbf{7}($ TripON)) crystallizes in the triclinic space group $P 1$ with one molecule of x8_16064 and two molecules of diethyl ether per asymmetric unit. One of the two ether molecules is coordinating to the Mo atom, the other one fills a void in the structure. Except for the ethylene molecule binding side-on to the molybdenum atom, all ligands show disorder. The non-coordinating ether molecule was refined as disordered over five positions, three of which are crystallographically unique. All disorders were refined as described above. The residual density map contains one peak that is significantly higher than the other residual electron density maxima (the highest peak corresponds to 2.31 electrons, the second highest peak to 0.91 electrons). This electron density maximum is located $0.73 \AA$ away from the molybdenum atom and may indicate whole-molecule disorder; however a model containing whole-molecule disorder could not be established. 
Table S5. Crystal data and structure refinement for X16005_t4 (2(MesON)).

Identification code

Empirical formula

Formula weight

Temperature

Wavelength

Crystal system

Space group

Unit cell dimensions

Volume

Z

Density (calculated)

Absorption coefficient

$F(000)$

Crystal size

Theta range for data collection

Index ranges

Reflections collected

Independent reflections

Completeness to theta $=25.242^{\circ}$

Absorption correction

Max. and min. transmission

Refinement method

Data / restraints / parameters

Goodness-of-fit on $F^{2}$

Final $R$ indices $[I>2 \sigma(I)]$

$R$ indices (all data)

Largest diff. peak and hole
X8_16005

$\mathrm{C}_{44} \mathrm{H}_{40} \mathrm{Cl} \mathrm{F}_{5} \mathrm{Mo} \mathrm{N}_{2} \mathrm{O}$

839.17

100(2) K

$0.71073 \AA$

Monoclinic

$P 2_{1} / n$

$a=20.4635(18) \AA$

$?=90^{\circ}$

$b=16.5228(14) \AA$

? $=104.780(2)^{\circ}$

$c=23.384(2) \AA$

? $=90^{\circ}$

$7644.8(12) \AA^{3}$

8

$1.458 \mathrm{Mg} / \mathrm{m}^{3}$

$0.475 \mathrm{~mm}^{-1}$

3440

$0.385 \times 0.250 \times 0.235 \mathrm{~mm}^{3}$

1.526 to $29.574^{\circ}$.

$-29<=h<=28,0<=k<=23,0<=k<=33$

21221

$21221\left[R_{\text {int }}=0.0913\right]$

$99.7 \%$

Semi-empirical from equivalents

0.746070 and 0.542269

Full-matrix least-squares on $F^{2}$

21221 / 406 / 1068

1.034

$R 1=0.0479, w R 2=0.1062$

$R 1=0.0682, w R 2=0.1133$

0.770 and -1.372 e. $\AA^{-3}$ 
Table S6. Bond lengths $[\AA ̊]$ and angles [ $\left[^{\circ}\right]$ for X16005_t4 (2(MesON)).

\begin{tabular}{|c|c|c|c|}
\hline $\mathrm{Cl}(1)-\mathrm{Mo}(1)$ & $2.3875(6)$ & $C(31)-C(32)$ & $1.375(4)$ \\
\hline $\mathrm{Mo}(1)-\mathrm{N}(2)$ & $1.752(2)$ & $\mathrm{C}(31)-\mathrm{H}(31)$ & 0.9500 \\
\hline $\mathrm{Mo}(1)-\mathrm{C}(1)$ & $1.870(2)$ & $\mathrm{C}(32)-\mathrm{H}(32)$ & 0.9500 \\
\hline $\mathrm{Mo}(1)-\mathrm{O}(1)$ & $1.9880(17)$ & $C(33)-C(38)$ & $1.398(3)$ \\
\hline $\mathrm{Mo}(1)-\mathrm{N}(1)$ & $2.212(2)$ & $C(33)-C(34)$ & $1.411(3)$ \\
\hline$O(1)-C(21)$ & $1.349(3)$ & $C(34)-C(35)$ & $1.387(3)$ \\
\hline$N(1)-C(32)$ & $1.347(3)$ & $C(34)-C(39)$ & $1.507(3)$ \\
\hline $\mathrm{N}(1)-\mathrm{C}(28)$ & $1.349(3)$ & $C(35)-C(36)$ & $1.388(4)$ \\
\hline$N(2)-C(42)$ & $1.375(3)$ & $\mathrm{C}(35)-\mathrm{H}(35)$ & 0.9500 \\
\hline$C(1)-C(2)$ & $1.522(3)$ & $C(36)-C(37)$ & $1.389(4)$ \\
\hline $\mathrm{C}(1)-\mathrm{H}(1)$ & $0.957(17)$ & $C(36)-C(40)$ & $1.508(4)$ \\
\hline$C(2)-C(3)$ & $1.531(4)$ & $C(37)-C(38)$ & $1.394(4)$ \\
\hline$C(2)-C(5)$ & $1.536(4)$ & $\mathrm{C}(37)-\mathrm{H}(37)$ & 0.9500 \\
\hline$C(2)-C(4)$ & $1.537(4)$ & $C(38)-C(41)$ & $1.509(4)$ \\
\hline$C(3)-H(3 A)$ & 0.9800 & $C(39)-H(39 A)$ & 0.9800 \\
\hline $\mathrm{C}(3)-\mathrm{H}(3 \mathrm{~B})$ & 0.9800 & $C(39)-H(39 B)$ & 0.9800 \\
\hline$C(3)-H(3 C)$ & 0.9800 & $C(39)-H(39 C)$ & 0.9800 \\
\hline $\mathrm{C}(4)-\mathrm{H}(4 \mathrm{~A})$ & 0.9800 & $\mathrm{C}(40)-\mathrm{H}(40 \mathrm{~A})$ & 0.9800 \\
\hline$C(4)-H(4 B)$ & 0.9800 & $\mathrm{C}(40)-\mathrm{H}(40 \mathrm{~B})$ & 0.9800 \\
\hline$C(4)-H(4 C)$ & 0.9800 & $C(40)-H(40 C)$ & 0.9800 \\
\hline$C(5)-C(6)$ & $1.393(4)$ & $C(41)-H(41 A)$ & 0.9800 \\
\hline$C(5)-C(10)$ & $1.397(4)$ & $\mathrm{C}(41)-\mathrm{H}(41 \mathrm{~B})$ & 0.9800 \\
\hline$C(6)-C(7)$ & $1.388(4)$ & $\mathrm{C}(41)-\mathrm{H}(41 \mathrm{C})$ & 0.9800 \\
\hline $\mathrm{C}(6)-\mathrm{H}(6)$ & 0.9500 & $C(42)-C(47)$ & $1.395(4)$ \\
\hline$C(7)-C(8)$ & $1.387(4)$ & $C(42)-C(43)$ & $1.403(4)$ \\
\hline $\mathrm{C}(7)-\mathrm{H}(7)$ & 0.9500 & $C(43)-F(1)$ & $1.336(3)$ \\
\hline$C(8)-C(9)$ & $1.384(4)$ & $C(43)-C(44)$ & $1.374(4)$ \\
\hline $\mathrm{C}(8)-\mathrm{H}(8)$ & 0.9500 & $C(44)-F(2)$ & $1.337(3)$ \\
\hline$C(9)-C(10)$ & $1.385(4)$ & $C(44)-C(45)$ & $1.377(5)$ \\
\hline $\mathrm{C}(9)-\mathrm{H}(9)$ & 0.9500 & $C(45)-F(3)$ & $1.341(3)$ \\
\hline$C(10)-H(10)$ & 0.9500 & $C(45)-C(46)$ & $1.373(5)$ \\
\hline$C(21)-C(26)$ & $1.406(3)$ & $C(46)-F(4)$ & $1.343(4)$ \\
\hline$C(21)-C(22)$ & $1.410(3)$ & $C(46)-C(47)$ & $1.379(4)$ \\
\hline$C(22)-C(23)$ & $1.402(3)$ & $C(47)-F(5)$ & $1.337(3)$ \\
\hline$C(22)-C(28)$ & $1.473(3)$ & $C(10 S)-C(11 S)$ & $1.496(5)$ \\
\hline$C(23)-C(24)$ & $1.382(3)$ & $C(10 S)-H(10 A)$ & 0.9800 \\
\hline $\mathrm{C}(23)-\mathrm{H}(23)$ & 0.9500 & $C(10 S)-H(10 B)$ & 0.9800 \\
\hline$C(24)-C(25)$ & $1.398(3)$ & $C(10 S)-H(10 C)$ & 0.9800 \\
\hline$C(24)-C(27)$ & $1.510(3)$ & $C(11 S)-C(12 S)$ & $1.389(4)$ \\
\hline$C(25)-C(26)$ & $1.391(3)$ & $C(11 S)-C(16 S)$ & $1.401(4)$ \\
\hline $\mathrm{C}(25)-\mathrm{H}(25)$ & 0.9500 & $C(12 S)-C(13 S)$ & $1.385(5)$ \\
\hline$C(26)-C(33)$ & $1.494(3)$ & $C(12 S)-H(12 S)$ & 0.9500 \\
\hline$C(27)-H(27 A)$ & 0.9800 & $C(13 S)-C(14 S)$ & $1.384(5)$ \\
\hline$C(27)-H(27 B)$ & 0.9800 & $C(13 S)-H(13 S)$ & 0.9500 \\
\hline $\mathrm{C}(27)-\mathrm{H}(27 \mathrm{C})$ & 0.9800 & $C(14 S)-C(15 S)$ & $1.388(5)$ \\
\hline$C(28)-C(29)$ & $1.398(3)$ & $\mathrm{C}(14 \mathrm{~S})-\mathrm{H}(14 \mathrm{~S})$ & 0.9500 \\
\hline$C(29)-C(30)$ & $1.378(3)$ & $C(15 S)-C(16 S)$ & $1.373(5)$ \\
\hline$C(29)-H(29)$ & 0.9500 & $C(15 S)-H(15 S)$ & 0.9500 \\
\hline$C(30)-C(31)$ & $1.388(4)$ & $C(16 S)-H(16 S)$ & 0.9500 \\
\hline$C(30)-H(30)$ & 0.9500 & $\mathrm{Cl}(2)-\mathrm{Mo}(2)$ & $2.3879(6$ \\
\hline
\end{tabular}




\begin{tabular}{|c|c|c|c|}
\hline$O(2)-C(61)$ & $1.357(3)$ & $C(62)-C(73)$ & $1.496(3)$ \\
\hline $\mathrm{O}(2)-\mathrm{Mo}(2)$ & $1.9812(17)$ & $C(63)-C(64)$ & $1.403(3)$ \\
\hline$N(3)-C(72)$ & $1.345(3)$ & $C(63)-H(63)$ & 0.9500 \\
\hline$N(3)-C(68)$ & $1.354(3)$ & $C(64)-C(65)$ & $1.383(3)$ \\
\hline $\mathrm{N}(3)-\mathrm{Mo}(2)$ & $2.203(2)$ & $C(64)-C(67)$ & $1.502(3)$ \\
\hline$N(4)-C(82)$ & $1.377(3)$ & $C(65)-C(66)$ & $1.400(3)$ \\
\hline $\mathrm{N}(4)-\mathrm{Mo}(2)$ & $1.755(2)$ & $C(65)-H(65)$ & 0.9500 \\
\hline $\mathrm{Mo}(2)-\mathrm{C}(51)$ & $1.869(3)$ & $C(66)-C(68)$ & $1.477(3)$ \\
\hline$C(51)-C(52)$ & $1.523(4)$ & $C(67)-H(67 A)$ & 0.9800 \\
\hline$C(51)-H(51)$ & $0.956(17)$ & $\mathrm{C}(67)-\mathrm{H}(67 \mathrm{~B})$ & 0.9800 \\
\hline$C(52)-C(53 A)$ & $1.517(10)$ & $\mathrm{C}(67)-\mathrm{H}(67 \mathrm{C})$ & 0.9800 \\
\hline$C(52)-C(54)$ & $1.533(9)$ & $C(68)-C(69)$ & $1.396(3)$ \\
\hline$C(52)-C(55)$ & $1.534(8)$ & $C(69)-C(70)$ & $1.385(4)$ \\
\hline$C(52)-C(55 A)$ & $1.541(9)$ & $C(69)-H(69)$ & 0.9500 \\
\hline$C(52)-C(53)$ & $1.548(9)$ & $C(70)-C(71)$ & $1.385(4)$ \\
\hline$C(52)-C(54 A)$ & $1.554(9)$ & $\mathrm{C}(70)-\mathrm{H}(70)$ & 0.9500 \\
\hline$C(53)-H(53 A)$ & 0.9800 & $C(71)-C(72)$ & $1.377(4)$ \\
\hline$C(53)-H(53 B)$ & 0.9800 & $\mathrm{C}(71)-\mathrm{H}(71)$ & 0.9500 \\
\hline$C(53)-H(53 C)$ & 0.9800 & $\mathrm{C}(72)-\mathrm{H}(72)$ & 0.9500 \\
\hline$C(54)-H(54 A)$ & 0.9800 & $C(73)-C(78)$ & $1.394(3)$ \\
\hline $\mathrm{C}(54)-\mathrm{H}(54 \mathrm{~B})$ & 0.9800 & $C(73)-C(74)$ & $1.410(3)$ \\
\hline $\mathrm{C}(54)-\mathrm{H}(54 \mathrm{C})$ & 0.9800 & $C(74)-C(75)$ & $1.390(3)$ \\
\hline$C(55)-C(60)$ & $1.388(9)$ & $C(74)-C(79)$ & $1.508(3)$ \\
\hline$C(55)-C(56)$ & $1.401(10)$ & $C(75)-C(76)$ & $1.391(3)$ \\
\hline$C(56)-C(57)$ & $1.388(9)$ & $C(75)-H(75)$ & 0.9500 \\
\hline$C(56)-H(56)$ & 0.9500 & $C(76)-C(77)$ & $1.390(4)$ \\
\hline$C(57)-C(58)$ & $1.370(10)$ & $C(76)-C(80)$ & $1.505(3)$ \\
\hline$C(57)-H(57)$ & 0.9500 & $C(77)-C(78)$ & $1.396(3)$ \\
\hline$C(58)-C(59)$ & $1.389(9)$ & $\mathrm{C}(77)-\mathrm{H}(77)$ & 0.9500 \\
\hline$C(58)-H(58)$ & 0.9500 & $C(78)-C(81)$ & $1.509(3)$ \\
\hline$C(59)-C(60)$ & $1.393(10)$ & $\mathrm{C}(79)-\mathrm{H}(79 \mathrm{~A})$ & 0.9800 \\
\hline $\mathrm{C}(59)-\mathrm{H}(59)$ & 0.9500 & $\mathrm{C}(79)-\mathrm{H}(79 \mathrm{~B})$ & 0.9800 \\
\hline$C(60)-H(60)$ & 0.9500 & $\mathrm{C}(79)-\mathrm{H}(79 \mathrm{C})$ & 0.9800 \\
\hline$C(53 A)-H(53 D)$ & 0.9800 & $\mathrm{C}(80)-\mathrm{H}(80 \mathrm{~A})$ & 0.9800 \\
\hline$C(53 A)-H(53 E)$ & 0.9800 & $\mathrm{C}(80)-\mathrm{H}(80 \mathrm{~B})$ & 0.9800 \\
\hline$C(53 A)-H(53 F)$ & 0.9800 & $C(80)-H(80 C)$ & 0.9800 \\
\hline$C(54 A)-H(54 D)$ & 0.9800 & $\mathrm{C}(81)-\mathrm{H}(81 \mathrm{~A})$ & 0.9800 \\
\hline$C(54 \mathrm{~A})-\mathrm{H}(54 \mathrm{E})$ & 0.9800 & $\mathrm{C}(81)-\mathrm{H}(81 \mathrm{~B})$ & 0.9800 \\
\hline$C(54 A)-H(54 F)$ & 0.9800 & $\mathrm{C}(81)-\mathrm{H}(81 \mathrm{C})$ & 0.9800 \\
\hline$C(55 A)-C(60 A)$ & $1.389(10)$ & $C(82)-C(83)$ & $1.392(3)$ \\
\hline$C(55 A)-C(56 A)$ & $1.403(10)$ & $C(82)-C(87)$ & $1.394(4)$ \\
\hline$C(56 A)-C(57 A)$ & $1.398(10)$ & $C(83)-F(6)$ & $1.337(3)$ \\
\hline$C(56 A)-H(56 A)$ & 0.9500 & $C(83)-C(84)$ & $1.373(4)$ \\
\hline$C(57 A)-C(58 A)$ & $1.366(10)$ & $C(84)-F(7)$ & $1.337(3)$ \\
\hline$C(57 A)-H(57 A)$ & 0.9500 & $C(84)-C(85)$ & $1.373(4)$ \\
\hline$C(58 A)-C(59 A)$ & $1.387(9)$ & $C(85)-F(8)$ & $1.341(3)$ \\
\hline$C(58 A)-H(58 A)$ & 0.9500 & $C(85)-C(86)$ & $1.372(4)$ \\
\hline$C(59 A)-C(60 A)$ & $1.385(9)$ & $C(86)-F(9)$ & $1.345(3)$ \\
\hline$C(59 A)-H(59 A)$ & 0.9500 & $C(86)-C(87)$ & $1.374(4)$ \\
\hline$C(60 A)-H(60 A)$ & 0.9500 & $C(87)-F(10)$ & $1.342(3)$ \\
\hline$C(61)-C(62)$ & $1.405(3)$ & $C(20 S)-C(21 S)$ & $1.493(5)$ \\
\hline$C(61)-C(66)$ & $1.408(3)$ & $C(20 S)-H(20 A)$ & 0.9800 \\
\hline$C(62)-C(63)$ & $1.387(3)$ & $C(20 S)-H(20 B)$ & 0.9800 \\
\hline
\end{tabular}




\begin{tabular}{|c|c|c|c|}
\hline$C(20 S)-H(20 C)$ & 0.9800 & $C(7)-C(6)-H(6)$ & 119.5 \\
\hline$C(21 S)-C(26 S)$ & $1.394(4)$ & $C(5)-C(6)-H(6)$ & 119.5 \\
\hline$C(21 S)-C(22 S)$ & $1.413(4)$ & $C(8)-C(7)-C(6)$ & $120.5(3)$ \\
\hline$C(22 S)-C(23 S)$ & $1.362(5)$ & $\mathrm{C}(8)-\mathrm{C}(7)-\mathrm{H}(7)$ & 119.7 \\
\hline$C(22 S)-H(22 S)$ & 0.9500 & $C(6)-C(7)-H(7)$ & 119.7 \\
\hline$C(23 S)-C(24 S)$ & $1.398(5)$ & $C(9)-C(8)-C(7)$ & $118.9(3)$ \\
\hline$C(23 S)-H(23 S)$ & 0.9500 & $C(9)-C(8)-H(8)$ & 120.5 \\
\hline$C(24 S)-C(25 S)$ & $1.383(5)$ & $C(7)-C(8)-H(8)$ & 120.5 \\
\hline$C(24 S)-H(24 S)$ & 0.9500 & $C(8)-C(9)-C(10)$ & $120.7(3)$ \\
\hline$C(25 S)-C(26 S)$ & $1.373(5)$ & $\mathrm{C}(8)-\mathrm{C}(9)-\mathrm{H}(9)$ & 119.7 \\
\hline$C(25 S)-H(25 S)$ & 0.9500 & $C(10)-C(9)-H(9)$ & 119.7 \\
\hline \multirow[t]{2}{*}{$\mathrm{C}(26 \mathrm{~S})-\mathrm{H}(26 \mathrm{~S})$} & 0.9500 & $C(9)-C(10)-C(5)$ & $121.0(3)$ \\
\hline & & $C(9)-C(10)-H(10)$ & 119.5 \\
\hline $\mathrm{N}(2)-\mathrm{Mo}(1)-\mathrm{C}(1)$ & $101.68(11)$ & $C(5)-C(10)-H(10)$ & 119.5 \\
\hline $\mathrm{N}(2)-\mathrm{Mo}(1)-\mathrm{O}(1)$ & $150.40(8)$ & $O(1)-C(21)-C(26)$ & $120.5(2)$ \\
\hline $\mathrm{C}(1)-\mathrm{Mo}(1)-\mathrm{O}(1)$ & $107.12(9)$ & $O(1)-C(21)-C(22)$ & $119.5(2)$ \\
\hline$N(2)-M o(1)-N(1)$ & $90.67(8)$ & $C(26)-C(21)-C(22)$ & $120.0(2)$ \\
\hline$C(1)-M o(1)-N(1)$ & $99.17(9)$ & $C(23)-C(22)-C(21)$ & $119.2(2)$ \\
\hline $\mathrm{O}(1)-\mathrm{Mo}(1)-\mathrm{N}(1)$ & $78.28(7)$ & $C(23)-C(22)-C(28)$ & $120.6(2)$ \\
\hline $\mathrm{N}(2)-\mathrm{Mo}(1)-\mathrm{Cl}(1)$ & $95.06(7)$ & $C(21)-C(22)-C(28)$ & $119.7(2)$ \\
\hline $\mathrm{C}(1)-\mathrm{Mo}(1)-\mathrm{Cl}(1)$ & $100.81(8)$ & $C(24)-C(23)-C(22)$ & $121.3(2)$ \\
\hline $\mathrm{O}(1)-\mathrm{Mo}(1)-\mathrm{Cl}(1)$ & $86.26(5)$ & $C(24)-C(23)-H(23)$ & 119.3 \\
\hline $\mathrm{N}(1)-\mathrm{Mo}(1)-\mathrm{Cl}(1)$ & $157.62(6)$ & $C(22)-C(23)-H(23)$ & 119.3 \\
\hline $\mathrm{C}(21)-\mathrm{O}(1)-\mathrm{Mo}(1)$ & 114.48(14) & $C(23)-C(24)-C(25)$ & $118.1(2)$ \\
\hline$C(32)-N(1)-C(28)$ & $119.2(2)$ & $C(23)-C(24)-C(27)$ & $121.1(2)$ \\
\hline$C(32)-N(1)-M o(1)$ & $122.24(16)$ & $C(25)-C(24)-C(27)$ & $120.7(2)$ \\
\hline $\mathrm{C}(28)-\mathrm{N}(1)-\mathrm{Mo}(1)$ & $118.55(15)$ & $C(26)-C(25)-C(24)$ & $122.7(2)$ \\
\hline $\mathrm{C}(42)-\mathrm{N}(2)-\mathrm{Mo}(1)$ & $165.35(19)$ & $C(26)-C(25)-H(25)$ & 118.7 \\
\hline$C(2)-C(1)-M o(1)$ & $142.8(2)$ & $C(24)-C(25)-H(25)$ & 118.7 \\
\hline$C(2)-C(1)-H(1)$ & $115.5(18)$ & $C(25)-C(26)-C(21)$ & $118.2(2)$ \\
\hline$M o(1)-C(1)-H(1)$ & $101.6(18)$ & $C(25)-C(26)-C(33)$ & $120.2(2)$ \\
\hline$C(1)-C(2)-C(3)$ & $110.6(2)$ & $C(21)-C(26)-C(33)$ & $121.5(2)$ \\
\hline$C(1)-C(2)-C(5)$ & $105.5(2)$ & $C(24)-C(27)-H(27 A)$ & 109.5 \\
\hline$C(3)-C(2)-C(5)$ & $112.4(2)$ & $C(24)-C(27)-H(27 B)$ & 109.5 \\
\hline$C(1)-C(2)-C(4)$ & $108.6(2)$ & $H(27 A)-C(27)-H(27 B)$ & 109.5 \\
\hline$C(3)-C(2)-C(4)$ & $108.4(2)$ & $C(24)-C(27)-H(27 C)$ & 109.5 \\
\hline$C(5)-C(2)-C(4)$ & $111.3(2)$ & $H(27 A)-C(27)-H(27 C)$ & 109.5 \\
\hline$C(2)-C(3)-H(3 A)$ & 109.5 & $H(27 B)-C(27)-H(27 C)$ & 109.5 \\
\hline$C(2)-C(3)-H(3 B)$ & 109.5 & $N(1)-C(28)-C(29)$ & $120.1(2)$ \\
\hline$H(3 A)-C(3)-H(3 B)$ & 109.5 & $N(1)-C(28)-C(22)$ & $120.2(2)$ \\
\hline$C(2)-C(3)-H(3 C)$ & 109.5 & $C(29)-C(28)-C(22)$ & $119.6(2)$ \\
\hline$H(3 A)-C(3)-H(3 C)$ & 109.5 & $C(30)-C(29)-C(28)$ & $120.7(2)$ \\
\hline $\mathrm{H}(3 \mathrm{~B})-\mathrm{C}(3)-\mathrm{H}(3 \mathrm{C})$ & 109.5 & $C(30)-C(29)-H(29)$ & 119.7 \\
\hline$C(2)-C(4)-H(4 A)$ & 109.5 & $C(28)-C(29)-H(29)$ & 119.7 \\
\hline$C(2)-C(4)-H(4 B)$ & 109.5 & $C(29)-C(30)-C(31)$ & $118.4(2)$ \\
\hline $\mathrm{H}(4 \mathrm{~A})-\mathrm{C}(4)-\mathrm{H}(4 \mathrm{~B})$ & 109.5 & $C(29)-C(30)-H(30)$ & 120.8 \\
\hline$C(2)-C(4)-H(4 C)$ & 109.5 & $C(31)-C(30)-H(30)$ & 120.8 \\
\hline $\mathrm{H}(4 \mathrm{~A})-\mathrm{C}(4)-\mathrm{H}(4 \mathrm{C})$ & 109.5 & $C(32)-C(31)-C(30)$ & $118.9(2)$ \\
\hline $\mathrm{H}(4 \mathrm{~B})-\mathrm{C}(4)-\mathrm{H}(4 \mathrm{C})$ & 109.5 & $C(32)-C(31)-H(31)$ & 120.6 \\
\hline$C(6)-C(5)-C(10)$ & $117.8(2)$ & $C(30)-C(31)-H(31)$ & 120.6 \\
\hline$C(6)-C(5)-C(2)$ & $119.9(2)$ & $N(1)-C(32)-C(31)$ & $122.8(2)$ \\
\hline$C(10)-C(5)-C(2)$ & $122.2(2)$ & $N(1)-C(32)-H(32)$ & 118.6 \\
\hline$C(7)-C(6)-C(5)$ & $121.0(3)$ & $C(31)-C(32)-H(32)$ & 118.6 \\
\hline
\end{tabular}




$\begin{array}{ll}C(38)-C(33)-C(34) & 119.8(2) \\ C(38)-C(33)-C(26) & 121.6(2) \\ C(34)-C(33)-C(26) & 118.5(2) \\ C(35)-C(34)-C(33) & 119.1(2) \\ C(35)-C(34)-C(39) & 119.4(2) \\ C(33)-C(34)-C(39) & 121.4(2) \\ C(34)-C(35)-C(36) & 121.9(2) \\ C(34)-C(35)-H(35) & 119.1 \\ C(36)-C(35)-H(35) & 119.1 \\ C(35)-C(36)-C(37) & 118.2(2) \\ C(35)-C(36)-C(40) & 120.6(2) \\ C(37)-C(36)-C(40) & 121.1(2) \\ C(36)-C(37)-C(38) & 121.8(2) \\ C(36)-C(37)-H(37) & 119.1 \\ C(38)-C(37)-H(37) & 119.1 \\ C(37)-C(38)-C(33) & 119.2(2) \\ C(37)-C(38)-C(41) & 119.3(2) \\ C(33)-C(38)-C(41) & 121.5(2) \\ C(34)-C(39)-H(39 A) & 109.5 \\ C(34)-C(39)-H(39 B) & 109.5 \\ H(39 A)-C(39)-H(39 B) & 109.5 \\ C(34)-C(39)-H(39 C) & 109.5 \\ H(39 A)-C(39)-H(39 C) & 109.5 \\ H(39 B)-C(39)-H(39 C) & 109.5 \\ C(36)-C(40)-H(40 A) & 109.5 \\ C(36)-C(40)-H(40 B) & 109.5 \\ H(40 A)-C(40)-H(40 B) & 109.5 \\ C(36)-C(40)-H(40 C) & 109.5 \\ H(40 A)-C(40)-H(40 C) & 109.5 \\ H(40 B)-C(40)-H(40 C) & 109.5 \\ C(38)-C(41)-H(41 A) & 109.5 \\ C(38)-C(41)-H(41 B) & 109.5 \\ H(41 A)-C(41)-H(41 B) & 109.5 \\ C(38)-C(41)-H(41 C) & 109.5 \\ H(41 A)-C(41)-H(41 C) & 109.5 \\ H(41 B)-C(41)-H(41 C) & 109.5 \\ N(2)-C(42)-C(47) & 122.5(2) \\ N(2)-C(42)-C(43) & 121.0(2) \\ C(47)-C(42)-C(43) & 116.5(2) \\ F(1)-C(43)-C(44) & 118.8(3) \\ F(1)-C(43)-C(42) & 119.3(2) \\ C(44)-C(43)-C(42) & 121.9(3) \\ F(2)-C(44)-C(43) & 120.2(3) \\ F(2)-C(44)-C(45) & 119.9(3) \\ C(43)-C(44)-C(45) & 119.9(3) \\ F(3)-C(45)-C(46) & 119.6(3) \\ F(3)-C(45)-C(44) & 120.5(3) \\ C(46)-C(45)-C(44) & 119.8(3) \\ F(4)-C(46)-C(45) & 120.5(3) \\ F(4)-C(46)-C(47) & 119.3(3) \\ C(45)-C(46)-C(47) & 120.2(3) \\ F(5)-C(47)-C(46) & 119.2(3) \\ F(5)-C(47)-C(42) & \\ & \end{array}$

\begin{tabular}{|c|c|}
\hline$C(46)-C(47)-C(42)$ & $121.6(3)$ \\
\hline$C(11 S)-C(10 S)-H(10 A)$ & 109.5 \\
\hline$C(11 S)-C(10 S)-H(10 B)$ & 109.5 \\
\hline$H(10 A)-C(10 S)-H(10 B)$ & 109.5 \\
\hline$C(11 S)-C(10 S)-H(10 C)$ & 109.5 \\
\hline$H(10 A)-C(10 S)-H(10 C)$ & 109.5 \\
\hline$H(10 B)-C(10 S)-H(10 C)$ & 109.5 \\
\hline$C(12 S)-C(11 S)-C(16 S)$ & $118.0(3)$ \\
\hline$C(12 S)-C(11 S)-C(10 S)$ & 121.3(3) \\
\hline$C(16 S)-C(11 S)-C(10 S)$ & $120.7(3)$ \\
\hline$C(13 S)-C(12 S)-C(11 S)$ & $121.3(3)$ \\
\hline$C(13 S)-C(12 S)-H(12 S)$ & 119.4 \\
\hline$C(11 S)-C(12 S)-H(12 S)$ & 119.4 \\
\hline$C(14 S)-C(13 S)-C(12 S)$ & $119.8(3)$ \\
\hline$C(14 S)-C(13 S)-H(13 S)$ & 120.1 \\
\hline$C(12 S)-C(13 S)-H(13 S)$ & 120.1 \\
\hline$C(13 S)-C(14 S)-C(15 S)$ & 119.7(3) \\
\hline$C(13 S)-C(14 S)-H(14 S)$ & 120.2 \\
\hline $\mathrm{C}(15 \mathrm{~S})-\mathrm{C}(14 \mathrm{~S})-\mathrm{H}(14 \mathrm{~S})$ & 120.2 \\
\hline$C(16 S)-C(15 S)-C(14 S)$ & $120.3(3)$ \\
\hline$C(16 S)-C(15 S)-H(15 S)$ & 119.9 \\
\hline$C(14 S)-C(15 S)-H(15 S)$ & 119.9 \\
\hline$C(15 S)-C(16 S)-C(11 S)$ & $121.0(3)$ \\
\hline$C(15 S)-C(16 S)-H(16 S)$ & 119.5 \\
\hline$C(11 S)-C(16 S)-H(16 S)$ & 119.5 \\
\hline $\mathrm{C}(61)-\mathrm{O}(2)-\mathrm{Mo}(2)$ & $113.93(14)$ \\
\hline$C(72)-N(3)-C(68)$ & $118.9(2)$ \\
\hline $\mathrm{C}(72)-\mathrm{N}(3)-\mathrm{Mo}(2)$ & $122.04(17)$ \\
\hline$C(68)-N(3)-M o(2)$ & 118.91(16) \\
\hline $\mathrm{C}(82)-\mathrm{N}(4)-\mathrm{Mo}(2)$ & $158.49(19)$ \\
\hline $\mathrm{N}(4)-\mathrm{Mo}(2)-\mathrm{C}(51)$ & $102.46(11)$ \\
\hline $\mathrm{N}(4)-\mathrm{Mo}(2)-\mathrm{O}(2)$ & $149.19(9)$ \\
\hline $\mathrm{C}(51)-\mathrm{Mo}(2)-\mathrm{O}(2)$ & $107.50(9)$ \\
\hline $\mathrm{N}(4)-\mathrm{Mo}(2)-\mathrm{N}(3)$ & 89.37(9) \\
\hline $\mathrm{C}(51)-\mathrm{Mo}(2)-\mathrm{N}(3)$ & $101.78(10)$ \\
\hline $\mathrm{O}(2)-\mathrm{Mo}(2)-\mathrm{N}(3)$ & 77.78(7) \\
\hline $\mathrm{N}(4)-\mathrm{Mo}(2)-\mathrm{Cl}(2)$ & $95.41(7)$ \\
\hline C(51)-Mo(2)-Cl(2) & $99.75(8)$ \\
\hline $\mathrm{O}(2)-\mathrm{Mo}(2)-\mathrm{Cl}(2)$ & $86.59(5)$ \\
\hline $\mathrm{N}(3)-\mathrm{Mo}(2)-\mathrm{Cl}(2)$ & $156.38(6)$ \\
\hline $\mathrm{C}(52)-\mathrm{C}(51)-\mathrm{Mo}(2)$ & $140.9(2)$ \\
\hline $\mathrm{C}(52)-\mathrm{C}(51)-\mathrm{H}(51)$ & $115.8(18)$ \\
\hline $\mathrm{Mo}(2)-\mathrm{C}(51)-\mathrm{H}(51)$ & $102.8(18)$ \\
\hline$C(53 A)-C(52)-C(51)$ & $111.6(10)$ \\
\hline$C(51)-C(52)-C(54)$ & 110.1(9) \\
\hline$C(51)-C(52)-C(55)$ & 105.9(9) \\
\hline$C(54)-C(52)-C(55)$ & 113.3(9) \\
\hline$C(53 A)-C(52)-C(55 A)$ & $113.5(10)$ \\
\hline$C(51)-C(52)-C(55 A)$ & $104.3(10)$ \\
\hline$C(51)-C(52)-C(53)$ & 108.6(9) \\
\hline$C(54)-C(52)-C(53)$ & $107.3(10)$ \\
\hline$C(55)-C(52)-C(53)$ & $111.5(9)$ \\
\hline$C(53 A)-C(52)-C(54 A)$ & $108.4(10)$ \\
\hline
\end{tabular}




$\begin{array}{ll} & \\ C(51)-C(52)-C(54 A) & 108.4(8) \\ C(55 A)-C(52)-C(54 A) & 110.4(10) \\ C(52)-C(53)-H(53 A) & 109.5 \\ C(52)-C(53)-H(53 B) & 109.5 \\ H(53 A)-C(53)-H(53 B) & 109.5 \\ C(52)-C(53)-H(53 C) & 109.5 \\ H(53 A)-C(53)-H(53 C) & 109.5 \\ H(53 B)-C(53)-H(53 C) & 109.5 \\ C(52)-C(54)-H(54 A) & 109.5 \\ C(52)-C(54)-H(54 B) & 109.5 \\ H(54 A)-C(54)-H(54 B) & 109.5 \\ C(52)-C(54)-H(54 C) & 109.5 \\ H(54 A)-C(54)-H(54 C) & 109.5 \\ H(54 B)-C(54)-H(54 C) & 109.5 \\ C(60)-C(55)-C(56) & 117.8(8) \\ C(60)-C(55)-C(52) & 123.9(9) \\ C(56)-C(55)-C(52) & 117.2(9) \\ C(57)-C(56)-C(55) & 119.9(8) \\ C(57)-C(56)-H(56) & 120.1 \\ C(55)-C(56)-H(56) & 120.1 \\ C(58)-C(57)-C(56) & 121.3(8) \\ C(58)-C(57)-H(57) & 119.3 \\ C(56)-C(57)-H(57) & 119.3 \\ C(57)-C(58)-C(59) & 120.0(8) \\ C(57)-C(58)-H(58) & 120.0 \\ C(59)-C(58)-H(58) & 120.0 \\ C(58)-C(59)-C(60) & 118.7(8) \\ C(58)-C(59)-H(59) & 120.7 \\ C(60)-C(59)-H(59) & 120.7 \\ C(55)-C(60)-C(59) & 122.2(9) \\ C(55)-C(60)-H(60) & 118.9 \\ C(59)-C(60)-H(60) & 118.9 \\ C(52)-C(53 A)-H(53 D) & 109.5 \\ C(52)-C(53 A)-H(53 E) & 109.5 \\ H(53 D)-C(53 A)-H(53 E) & 109.5 \\ C(52)-C(53 A)-H(53 F) & 109.5 \\ H(53 D)-C(53 A)-H(53 F) & 109.5 \\ H(53 E)-C(53 A)-H(53 F) & 109.5 \\ C(52)-C(54 A)-H(54 D) & 109.5 \\ C(52)-C(54 A)-H(54 E) & 109.5 \\ H(54 D)-C(54 A)-H(54 E) & 109.5 \\ C(52)-C(54 A)-H(54 F) & 109.5 \\ H(54 D)-C(54 A)-H(54 F) & 109.5 \\ H(54 E)-C(54 A)-H(54 F) & 109.5 \\ C(60 A)-C(55 A)-C(56 A) & 117.4(8) \\ C(60 A)-C(55 A)-C(52) & 120.5(9) \\ C(56 A)-C(55 A)-C(52) & 122.0(10) \\ C(57 A)-C(56 A)-C(55 A) & 121.3(9) \\ C(57 A)-C(56 A)-H(56 A) & 119.3 \\ C(55 A)-C(56 A)-H(56 A) & 119.3 \\ C(58 A)-C(57 A)-C(56 A) & 119.3(9) \\ C(58 A)-C(57 A)-H(57 A) & 120.4 \\ C(56 A)-C(57 A)-H(57 A) & 120.4 \\ & \\ & \end{array}$

\begin{tabular}{|c|c|}
\hline$C(57 A)-C(58 A)-C(59 A)$ & $120.7(8)$ \\
\hline$C(57 A)-C(58 A)-H(58 A)$ & 119.7 \\
\hline$C(59 A)-C(58 A)-H(58 A)$ & 119.7 \\
\hline$C(60 A)-C(59 A)-C(58 A)$ & $119.8(8)$ \\
\hline$C(60 A)-C(59 A)-H(59 A)$ & 120.1 \\
\hline$C(58 A)-C(59 A)-H(59 A)$ & 120.1 \\
\hline$C(59 A)-C(60 A)-C(55 A)$ & $121.4(9)$ \\
\hline$C(59 A)-C(60 A)-H(60 A)$ & 119.3 \\
\hline$C(55 A)-C(60 A)-H(60 A)$ & 119.3 \\
\hline$O(2)-C(61)-C(62)$ & $120.1(2)$ \\
\hline$O(2)-C(61)-C(66)$ & $119.8(2)$ \\
\hline$C(62)-C(61)-C(66)$ & $120.1(2)$ \\
\hline$C(63)-C(62)-C(61)$ & $118.2(2)$ \\
\hline$C(63)-C(62)-C(73)$ & $121.7(2)$ \\
\hline$C(61)-C(62)-C(73)$ & $119.9(2)$ \\
\hline$C(62)-C(63)-C(64)$ & $122.5(2)$ \\
\hline$C(62)-C(63)-H(63)$ & 118.8 \\
\hline$C(64)-C(63)-H(63)$ & 118.8 \\
\hline$C(65)-C(64)-C(63)$ & $118.0(2)$ \\
\hline$C(65)-C(64)-C(67)$ & $121.2(2)$ \\
\hline$C(63)-C(64)-C(67)$ & $120.9(2)$ \\
\hline$C(64)-C(65)-C(66)$ & $121.4(2)$ \\
\hline$C(64)-C(65)-H(65)$ & 119.3 \\
\hline$C(66)-C(65)-H(65)$ & 119.3 \\
\hline$C(65)-C(66)-C(61)$ & $119.1(2)$ \\
\hline$C(65)-C(66)-C(68)$ & $120.7(2)$ \\
\hline$C(61)-C(66)-C(68)$ & $119.8(2)$ \\
\hline$C(64)-C(67)-H(67 A)$ & 109.5 \\
\hline$C(64)-C(67)-H(67 B)$ & 109.5 \\
\hline$H(67 A)-C(67)-H(67 B)$ & 109.5 \\
\hline$C(64)-C(67)-H(67 C)$ & 109.5 \\
\hline$H(67 A)-C(67)-H(67 C)$ & 109.5 \\
\hline$H(67 B)-C(67)-H(67 C)$ & 109.5 \\
\hline$N(3)-C(68)-C(69)$ & $120.4(2)$ \\
\hline$N(3)-C(68)-C(66)$ & $119.4(2)$ \\
\hline$C(69)-C(68)-C(66)$ & $120.0(2)$ \\
\hline$C(70)-C(69)-C(68)$ & $120.2(2)$ \\
\hline$C(70)-C(69)-H(69)$ & 119.9 \\
\hline$C(68)-C(69)-H(69)$ & 119.9 \\
\hline$C(71)-C(70)-C(69)$ & $118.7(2)$ \\
\hline$C(71)-C(70)-H(70)$ & 120.7 \\
\hline$C(69)-C(70)-H(70)$ & 120.7 \\
\hline$C(72)-C(71)-C(70)$ & $118.7(2)$ \\
\hline$C(72)-C(71)-H(71)$ & 120.7 \\
\hline$C(70)-C(71)-H(71)$ & 120.7 \\
\hline$N(3)-C(72)-C(71)$ & $123.1(2)$ \\
\hline$N(3)-C(72)-H(72)$ & 118.4 \\
\hline$C(71)-C(72)-H(72)$ & 118.4 \\
\hline$C(78)-C(73)-C(74)$ & $120.1(2)$ \\
\hline$C(78)-C(73)-C(62)$ & $122.2(2)$ \\
\hline$C(74)-C(73)-C(62)$ & $117.7(2)$ \\
\hline$C(75)-C(74)-C(73)$ & $119.3(2)$ \\
\hline$C(75)-C(74)-C(79)$ & $119.3(2)$ \\
\hline
\end{tabular}




$\begin{array}{ll}C(73)-C(74)-C(79) & 121.4(2) \\ C(74)-C(75)-C(76) & 121.5(2) \\ C(74)-C(75)-H(75) & 119.3 \\ C(76)-C(75)-H(75) & 119.3 \\ C(77)-C(76)-C(75) & 118.3(2) \\ C(77)-C(76)-C(80) & 120.9(2) \\ C(75)-C(76)-C(80) & 120.7(2) \\ C(76)-C(77)-C(78) & 121.9(2) \\ C(76)-C(77)-H(77) & 119.0 \\ C(78)-C(77)-H(77) & 119.0 \\ C(73)-C(78)-C(77) & 118.9(2) \\ C(73)-C(78)-C(81) & 121.8(2) \\ C(77)-C(78)-C(81) & 119.2(2) \\ C(74)-C(79)-H(79 A) & 109.5 \\ C(74)-C(79)-H(79 B) & 109.5 \\ H(79 A)-C(79)-H(79 B) & 109.5 \\ C(74)-C(79)-H(79 C) & 109.5 \\ H(79 A)-C(79)-H(79 C) & 109.5 \\ H(79 B)-C(79)-H(79 C) & 109.5 \\ C(76)-C(80)-H(80 A) & 109.5 \\ C(76)-C(80)-H(80 B) & 109.5 \\ H(80 A)-C(80)-H(80 B) & 109.5 \\ C(76)-C(80)-H(80 C) & 109.5 \\ H(80 A)-C(80)-H(80 C) & 109.5 \\ H(80 B)-C(80)-H(80 C) & 109.5 \\ C(78)-C(81)-H(81 A) & 109.5 \\ C(78)-C(81)-H(81 B) & 109.5 \\ H(81 A)-C(81)-H(81 B) & 109.5 \\ C(78)-C(81)-H(81 C) & 109.5 \\ H(81 A)-C(81)-H(81 C) & 109.5 \\ H(81 B)-C(81)-H(81 C) & 109.5 \\ N(4)-C(82)-C(83) & 121.3(2) \\ N(4)-C(82)-C(87) & 122.8(2) \\ C(83)-C(82)-C(87) & 115.9(2) \\ F(6)-C(83)-C(84) & 118.8(2) \\ F(6)-C(83)-C(82) & 119.0(2) \\ C(84)-C(83)-C(82) & 122.3(2) \\ & \\ & \\ & \end{array}$

$\begin{array}{ll}F(7)-C(84)-C(85) & 119.1(2) \\ F(7)-C(84)-C(83) & 120.7(2) \\ C(85)-C(84)-C(83) & 120.2(2) \\ F(8)-C(85)-C(86) & 119.9(3) \\ F(8)-C(85)-C(84) & 120.8(2) \\ C(86)-C(85)-C(84) & 119.2(2) \\ F(9)-C(86)-C(85) & 119.9(3) \\ F(9)-C(86)-C(87) & 119.8(3) \\ C(85)-C(86)-C(87) & 120.3(3) \\ F(10)-C(87)-C(86) & 118.7(2) \\ F(10)-C(87)-C(82) & 119.2(2) \\ C(86)-C(87)-C(82) & 122.1(3) \\ C(21 S)-C(20 S)-H(20 A) & 109.5 \\ C(21 S)-C(20 S)-H(20 B) & 109.5 \\ H(20 A)-C(20 S)-H(20 B) & 109.5 \\ C(21 S)-C(20 S)-H(20 C) & 109.5 \\ H(20 A)-C(20 S)-H(20 C) & 109.5 \\ H(20 B)-C(20 S)-H(20 C) & 109.5 \\ C(26 S)-C(21 S)-C(22 S) & 117.4(3) \\ C(26 S)-C(21 S)-C(20 S) & 122.3(3) \\ C(22 S)-C(21 S)-C(20 S) & 120.3(3) \\ C(23 S)-C(22 S)-C(21 S) & 120.7(3) \\ C(23 S)-C(22 S)-H(22 S) & 119.7 \\ C(21 S)-C(22 S)-H(22 S) & 119.7 \\ C(22 S)-C(23 S)-C(24 S) & 121.3(3) \\ C(22 S)-C(23 S)-H(23 S) & 119.3 \\ C(24 S)-C(23 S)-H(23 S) & 119.3 \\ C(25 S)-C(24 S)-C(23 S) & 118.3(3) \\ C(25 S)-C(24 S)-H(24 S) & 120.8 \\ C(23 S)-C(24 S)-H(24 S) & 120.8 \\ C(26 S)-C(25 S)-C(24 S) & 120.8(3) \\ C(26 S)-C(25 S)-H(25 S) & 119.6 \\ C(24 S)-C(25 S)-H(25 S) & 119.6 \\ C(25 S)-C(26 S)-C(21 S) & 121.5(3) \\ C(25 S)-C(26 S)-H(26 S) & 119.3 \\ C(21 S)-C(26 S)-H(26 S) & 119.3 \\ & \\ & \end{array}$


Table S7. Crystal data and structure refinement for X16023 (3(TripON)).

Identification code

Empirical formula

Formula weight

Temperature

Wavelength

Crystal system

Space group

Unit cell dimensions

Volume

Z

Density (calculated)

Absorption coefficient

$F(000)$

Crystal size

Theta range for data collection

Index ranges

Reflections collected

Independent reflections

Completeness to theta $=25.242^{\circ}$

Absorption correction

Refinement method

Data / restraints / parameters

Goodness-of-fit on $F^{2}$

Final $R$ indices $[/>2 \sigma(I)]$

$R$ indices (all data)

Largest diff. peak and hole
$\mathrm{X} 16023$

$\mathrm{C}_{50} \mathrm{H}_{65} \mathrm{Cl} \mathrm{Mo} \mathrm{N} \mathrm{O}$

841.43

100(2) K

$0.71073 \AA$

Monoclinic

$\mathrm{C} 2 / \mathrm{c}$

$a=39.702(3) \AA$

$b=12.5644(9) \AA$

$c=20.3447(15) \AA$

9088.5(12) $\AA^{3}$

8

$1.230 \mathrm{Mg} / \mathrm{m}^{3}$

$0.384 \mathrm{~mm}^{-1}$

3568

$0.220 \times 0.185 \times 0.125 \mathrm{~mm}^{3}$

1.145 to $30.508^{\circ}$.

$-56<=h<=56,-16<=k<=17,-29<=k<=29$

77710

$13859\left[R_{\text {int }}=0.0478\right]$

$100.0 \%$

Semi-empirical from equivalents

Full-matrix least-squares on $F^{2}$

13859 / 182 / 556

1.035

$R 1=0.0337, w R 2=0.0779$

$R 1=0.0438, w R 2=0.0823$

0.750 and -0.743 e. $\AA^{-3}$
] $=90^{\circ}$

[ $=116.4221(12)^{\circ}$

] $=90^{\circ}$ 
Table S8. Bond lengths $[\AA ̊ A]$ and angles $\left[\left[^{\circ}\right]\right.$ for $\mathbf{X 1 6 0 2 3 . ~}$

\begin{tabular}{|c|c|c|c|}
\hline $\mathrm{Cl}(1)-\mathrm{Mo}(1)$ & $2.4140(4)$ & $C(22)-C(28)$ & $1.473(2)$ \\
\hline $\mathrm{Mo}(1)-\mathrm{N}(1)$ & $1.7428(13)$ & $C(23)-C(24)$ & $1.379(2)$ \\
\hline$M o(1)-C(1)$ & $1.9082(14)$ & $C(23)-H(23)$ & 0.9500 \\
\hline $\mathrm{Mo}(1)-\mathrm{O}(1)$ & $1.9824(10)$ & $C(24)-C(25)$ & $1.396(2)$ \\
\hline $\mathrm{Mo}(1)-\mathrm{N}(2)$ & $2.2286(13)$ & $C(24)-C(27)$ & $1.514(2)$ \\
\hline$C(1)-C(2)$ & $1.526(2)$ & $C(25)-C(26)$ & $1.390(2)$ \\
\hline$C(1)-H(1)$ & $0.958(14)$ & $\mathrm{C}(25)-\mathrm{H}(25)$ & 0.9500 \\
\hline$C(2)-C(5)$ & $1.538(2)$ & $C(26)-C(33)$ & $1.509(2)$ \\
\hline$C(2)-C(4)$ & $1.538(2)$ & $C(27)-H(27 A)$ & 0.9800 \\
\hline$C(2)-C(3)$ & $1.544(2)$ & $\mathrm{C}(27)-\mathrm{H}(27 \mathrm{~B})$ & 0.9800 \\
\hline$C(3)-H(3 A)$ & 0.9800 & $\mathrm{C}(27)-\mathrm{H}(27 \mathrm{C})$ & 0.9800 \\
\hline$C(3)-H(3 B)$ & 0.9800 & $C(28)-C(29)$ & $1.405(2)$ \\
\hline$C(3)-H(3 C)$ & 0.9800 & $C(29)-C(30)$ & $1.379(2)$ \\
\hline$C(4)-H(4 A)$ & 0.9800 & $C(29)-H(29)$ & 0.9500 \\
\hline $\mathrm{C}(4)-\mathrm{H}(4 \mathrm{~B})$ & 0.9800 & $C(30)-C(31)$ & $1.383(3)$ \\
\hline $\mathrm{C}(4)-\mathrm{H}(4 \mathrm{C})$ & 0.9800 & $C(30)-H(30)$ & 0.9500 \\
\hline$C(5)-C(6)$ & $1.390(2)$ & $C(31)-C(32)$ & $1.369(3)$ \\
\hline$C(5)-C(10)$ & $1.396(2)$ & $C(31)-H(31)$ & 0.9500 \\
\hline$C(6)-C(7)$ & $1.391(2)$ & $\mathrm{C}(32)-\mathrm{H}(32)$ & 0.9500 \\
\hline$C(6)-H(6)$ & 0.9500 & $C(33)-C(38)$ & $1.404(2)$ \\
\hline$C(7)-C(8)$ & $1.389(3)$ & $C(33)-C(34)$ & $1.408(2)$ \\
\hline $\mathrm{C}(7)-\mathrm{H}(7)$ & 0.9500 & $C(34)-C(35)$ & $1.397(2)$ \\
\hline$C(8)-C(9)$ & $1.379(3)$ & $C(34)-C(39)$ & $1.525(2)$ \\
\hline $\mathrm{C}(8)-\mathrm{H}(8)$ & 0.9500 & $C(35)-C(36)$ & $1.394(2)$ \\
\hline$C(9)-C(10)$ & $1.385(2)$ & $C(35)-H(35)$ & 0.9500 \\
\hline $\mathrm{C}(9)-\mathrm{H}(9)$ & 0.9500 & $C(36)-C(37)$ & $1.383(2)$ \\
\hline$C(10)-H(10)$ & 0.9500 & $C(36)-C(42)$ & $1.523(2)$ \\
\hline$N(1)-C(11)$ & $1.3885(18)$ & $C(37)-C(38)$ & $1.398(2)$ \\
\hline$C(11)-C(12)$ & $1.406(3)$ & $\mathrm{C}(37)-\mathrm{H}(37)$ & 0.9500 \\
\hline$C(11)-C(16)$ & $1.412(2)$ & $C(38)-C(45)$ & $1.521(2)$ \\
\hline$C(12)-C(13)$ & $1.394(2)$ & $C(39)-C(40)$ & $1.525(2)$ \\
\hline$C(12)-C(17)$ & $1.508(3)$ & $C(39)-C(41)$ & $1.533(2)$ \\
\hline$C(13)-C(14)$ & $1.385(3)$ & $\mathrm{C}(39)-\mathrm{H}(39)$ & 1.0000 \\
\hline$C(13)-H(13)$ & 0.9500 & $\mathrm{C}(40)-\mathrm{H}(40 \mathrm{~A})$ & 0.9800 \\
\hline$C(14)-C(15)$ & $1.376(3)$ & $\mathrm{C}(40)-\mathrm{H}(40 \mathrm{~B})$ & 0.9800 \\
\hline $\mathrm{C}(14)-\mathrm{H}(14)$ & 0.9500 & $\mathrm{C}(40)-\mathrm{H}(40 \mathrm{C})$ & 0.9800 \\
\hline$C(15)-C(16)$ & $1.396(2)$ & $C(41)-H(41 A)$ & 0.9800 \\
\hline $\mathrm{C}(15)-\mathrm{H}(15)$ & 0.9500 & $\mathrm{C}(41)-\mathrm{H}(41 \mathrm{~B})$ & 0.9800 \\
\hline$C(16)-C(18)$ & $1.498(3)$ & $\mathrm{C}(41)-\mathrm{H}(41 \mathrm{C})$ & 0.9800 \\
\hline$C(17)-H(17 A)$ & 0.9800 & $C(42)-C(44)$ & $1.523(2)$ \\
\hline$C(17)-H(17 B)$ & 0.9800 & $C(42)-C(43)$ & $1.524(2)$ \\
\hline$C(17)-H(17 C)$ & 0.9800 & $\mathrm{C}(42)-\mathrm{H}(42)$ & 1.0000 \\
\hline$C(18)-H(18 A)$ & 0.9800 & $\mathrm{C}(43)-\mathrm{H}(43 \mathrm{~A})$ & 0.9800 \\
\hline$C(18)-H(18 B)$ & 0.9800 & $\mathrm{C}(43)-\mathrm{H}(43 \mathrm{~B})$ & 0.9800 \\
\hline$C(18)-H(18 C)$ & 0.9800 & $\mathrm{C}(43)-\mathrm{H}(43 \mathrm{C})$ & 0.9800 \\
\hline$N(2)-C(28)$ & $1.3567(19)$ & $\mathrm{C}(44)-\mathrm{H}(44 \mathrm{~A})$ & 0.9800 \\
\hline$N(2)-C(32)$ & $1.358(2)$ & $\mathrm{C}(44)-\mathrm{H}(44 \mathrm{~B})$ & 0.9800 \\
\hline$C(21)-O(1)$ & $1.3422(16)$ & $\mathrm{C}(44)-\mathrm{H}(44 \mathrm{C})$ & 0.9800 \\
\hline$C(21)-C(26)$ & $1.408(2)$ & $C(45)-C(46)$ & $1.529(2)$ \\
\hline$C(21)-C(22)$ & $1.411(2)$ & $C(45)-C(47)$ & $1.534(3)$ \\
\hline$C(22)-C(23)$ & $1.4054(19)$ & $\mathrm{C}(45)-\mathrm{H}(45)$ & 1.0000 \\
\hline
\end{tabular}




\begin{tabular}{|c|c|c|c|}
\hline$C(46)-H(46 A)$ & 0.9800 & $C(1)-C(2)-C(4)$ & $112.33(12)$ \\
\hline $\mathrm{C}(46)-\mathrm{H}(46 \mathrm{~B})$ & 0.9800 & $C(5)-C(2)-C(4)$ & $111.87(12)$ \\
\hline $\mathrm{C}(46)-\mathrm{H}(46 \mathrm{C})$ & 0.9800 & $C(1)-C(2)-C(3)$ & $107.32(12)$ \\
\hline$C(47)-H(47 A)$ & 0.9800 & $C(5)-C(2)-C(3)$ & $110.80(13)$ \\
\hline$C(47)-H(47 B)$ & 0.9800 & $C(4)-C(2)-C(3)$ & $108.30(13)$ \\
\hline $\mathrm{C}(47)-\mathrm{H}(47 \mathrm{C})$ & 0.9800 & $C(2)-C(3)-H(3 A)$ & 109.5 \\
\hline$C(1 S)-C(2 S)$ & $1.518(6)$ & $C(2)-C(3)-H(3 B)$ & 109.5 \\
\hline$C(1 S)-H(1 S 1)$ & 0.9800 & $H(3 A)-C(3)-H(3 B)$ & 109.5 \\
\hline$C(1 S)-H(1 S 2)$ & 0.9800 & $C(2)-C(3)-H(3 C)$ & 109.5 \\
\hline$C(1 S)-H(1 S 3)$ & 0.9800 & $H(3 A)-C(3)-H(3 C)$ & 109.5 \\
\hline$C(2 S)-C(3 S)$ & $1.492(6)$ & $H(3 B)-C(3)-H(3 C)$ & 109.5 \\
\hline $\mathrm{C}(2 \mathrm{~S})-\mathrm{H}(2 \mathrm{~S} 1)$ & 0.9900 & $C(2)-C(4)-H(4 A)$ & 109.5 \\
\hline $\mathrm{C}(2 \mathrm{~S})-\mathrm{H}(2 \mathrm{~S} 2)$ & 0.9900 & $\mathrm{C}(2)-\mathrm{C}(4)-\mathrm{H}(4 \mathrm{~B})$ & 109.5 \\
\hline$C(3 S)-C(4 S)$ & $1.514(6)$ & $H(4 A)-C(4)-H(4 B)$ & 109.5 \\
\hline$C(3 S)-H(3 S 1)$ & 0.9900 & $C(2)-C(4)-H(4 C)$ & 109.5 \\
\hline$C(3 S)-H(3 S 2)$ & 0.9900 & $H(4 A)-C(4)-H(4 C)$ & 109.5 \\
\hline$C(4 S)-C(5 S)$ & $1.538(7)$ & $\mathrm{H}(4 \mathrm{~B})-\mathrm{C}(4)-\mathrm{H}(4 \mathrm{C})$ & 109.5 \\
\hline$C(4 S)-H(4 S 1)$ & 0.9900 & $C(6)-C(5)-C(10)$ & $117.61(15)$ \\
\hline $\mathrm{C}(4 \mathrm{~S})-\mathrm{H}(4 \mathrm{~S} 2)$ & 0.9900 & $C(6)-C(5)-C(2)$ & $121.62(14)$ \\
\hline$C(5 S)-H(5 S 1)$ & 0.9800 & $C(10)-C(5)-C(2)$ & $120.71(14)$ \\
\hline$C(5 S)-H(5 S 2)$ & 0.9800 & $C(5)-C(6)-C(7)$ & $121.28(16)$ \\
\hline$C(5 S)-H(5 S 3)$ & 0.9800 & $C(5)-C(6)-H(6)$ & 119.4 \\
\hline$C(1 T)-C(2 T)$ & $1.529(13)$ & $C(7)-C(6)-H(6)$ & 119.4 \\
\hline $\mathrm{C}(1 \mathrm{~T})-\mathrm{H}(1 \mathrm{~T} 1)$ & 0.9800 & $C(8)-C(7)-C(6)$ & $120.25(17)$ \\
\hline $\mathrm{C}(1 \mathrm{~T})-\mathrm{H}(1 \mathrm{~T} 2)$ & 0.9800 & $\mathrm{C}(8)-\mathrm{C}(7)-\mathrm{H}(7)$ & 119.9 \\
\hline $\mathrm{C}(1 \mathrm{~T})-\mathrm{H}(1 \mathrm{~T} 3)$ & 0.9800 & $\mathrm{C}(6)-\mathrm{C}(7)-\mathrm{H}(7)$ & 119.9 \\
\hline$C(2 T)-C(3 T)$ & $1.466(13)$ & $C(9)-C(8)-C(7)$ & $118.97(16)$ \\
\hline $\mathrm{C}(2 \mathrm{~T})-\mathrm{H}(2 \mathrm{~T} 1)$ & 0.9900 & $C(9)-C(8)-H(8)$ & 120.5 \\
\hline $\mathrm{C}(2 \mathrm{~T})-\mathrm{H}(2 \mathrm{~T} 2)$ & 0.9900 & $C(7)-C(8)-H(8)$ & 120.5 \\
\hline$C(3 T)-C(4 T)$ & $1.471(12)$ & $C(8)-C(9)-C(10)$ & $120.73(17)$ \\
\hline $\mathrm{C}(3 \mathrm{~T})-\mathrm{H}(3 \mathrm{~T} 1)$ & 0.9900 & $\mathrm{C}(8)-\mathrm{C}(9)-\mathrm{H}(9)$ & 119.6 \\
\hline $\mathrm{C}(3 \mathrm{~T})-\mathrm{H}(3 \mathrm{~T} 2)$ & 0.9900 & $\mathrm{C}(10)-\mathrm{C}(9)-\mathrm{H}(9)$ & 119.6 \\
\hline$C(4 T)-C(5 T)$ & $1.506(12)$ & $C(9)-C(10)-C(5)$ & $121.14(17)$ \\
\hline $\mathrm{C}(4 \mathrm{~T})-\mathrm{H}(4 \mathrm{~T} 1)$ & 0.9900 & $C(9)-C(10)-H(10)$ & 119.4 \\
\hline $\mathrm{C}(4 \mathrm{~T})-\mathrm{H}(4 \mathrm{~T} 2)$ & 0.9900 & $C(5)-C(10)-H(10)$ & 119.4 \\
\hline$C(5 T)-H(5 T 1)$ & 0.9800 & $\mathrm{C}(11)-\mathrm{N}(1)-\mathrm{Mo}(1)$ & $156.70(12)$ \\
\hline$C(5 T)-H(5 T 2)$ & 0.9800 & $N(1)-C(11)-C(12)$ & $118.14(14)$ \\
\hline \multirow[t]{2}{*}{$\mathrm{C}(5 \mathrm{~T})-\mathrm{H}(5 \mathrm{~T} 3)$} & 0.9800 & $N(1)-C(11)-C(16)$ & 119.64(16) \\
\hline & & $C(12)-C(11)-C(16)$ & $122.22(15)$ \\
\hline $\mathrm{N}(1)-\mathrm{Mo}(1)-\mathrm{C}(1)$ & $100.05(6)$ & $C(13)-C(12)-C(11)$ & $117.57(17)$ \\
\hline $\mathrm{N}(1)-\mathrm{Mo}(1)-\mathrm{O}(1)$ & $141.39(5)$ & $C(13)-C(12)-C(17)$ & $122.24(18)$ \\
\hline$C(1)-M o(1)-O(1)$ & $116.84(5)$ & $C(11)-C(12)-C(17)$ & $120.19(14)$ \\
\hline $\mathrm{N}(1)-\mathrm{Mo}(1)-\mathrm{N}(2)$ & $87.08(5)$ & $C(14)-C(13)-C(12)$ & $121.0(2)$ \\
\hline$C(1)-M o(1)-N(2)$ & $91.47(6)$ & $C(14)-C(13)-H(13)$ & 119.5 \\
\hline $\mathrm{O}(1)-\mathrm{Mo}(1)-\mathrm{N}(2)$ & $81.26(4)$ & $\mathrm{C}(12)-\mathrm{C}(13)-\mathrm{H}(13)$ & 119.5 \\
\hline $\mathrm{N}(1)-\mathrm{Mo}(1)-\mathrm{Cl}(1)$ & $97.44(4)$ & $C(15)-C(14)-C(13)$ & $120.59(16)$ \\
\hline$C(1)-M o(1)-C l(1)$ & $100.37(5)$ & $\mathrm{C}(15)-\mathrm{C}(14)-\mathrm{H}(14)$ & 119.7 \\
\hline $\mathrm{O}(1)-\mathrm{Mo}(1)-\mathrm{Cl}(1)$ & $87.27(3)$ & $\mathrm{C}(13)-\mathrm{C}(14)-\mathrm{H}(14)$ & 119.7 \\
\hline $\mathrm{N}(2)-\mathrm{Mo}(1)-\mathrm{Cl}(1)$ & $166.34(3)$ & $C(14)-C(15)-C(16)$ & $121.25(18)$ \\
\hline$C(2)-C(1)-M o(1)$ & $127.55(11)$ & $C(14)-C(15)-H(15)$ & 119.4 \\
\hline$C(2)-C(1)-H(1)$ & $112.5(11)$ & $C(16)-C(15)-H(15)$ & 119.4 \\
\hline $\mathrm{Mo}(1)-\mathrm{C}(1)-\mathrm{H}(1)$ & $119.8(10)$ & $C(15)-C(16)-C(11)$ & $117.30(18)$ \\
\hline$C(1)-C(2)-C(5)$ & $106.14(12)$ & $C(15)-C(16)-C(18)$ & $121.93(16)$ \\
\hline
\end{tabular}




$\begin{array}{ll}C(11)-C(16)-C(18) & 120.77(14) \\ C(12)-C(17)-H(17 A) & 109.5 \\ C(12)-C(17)-H(17 B) & 109.5 \\ H(17 A)-C(17)-H(17 B) & 109.5 \\ C(12)-C(17)-H(17 C) & 109.5 \\ H(17 A)-C(17)-H(17 C) & 109.5 \\ H(17 B)-C(17)-H(17 C) & 109.5 \\ C(16)-C(18)-H(18 A) & 109.5 \\ C(16)-C(18)-H(18 B) & 109.5 \\ H(18 A)-C(18)-H(18 B) & 109.5 \\ C(16)-C(18)-H(18 C) & 109.5 \\ H(18 A)-C(18)-H(18 C) & 109.5 \\ H(18 B)-C(18)-H(18 C) & 109.5 \\ C(28)-N(2)-C(32) & 118.34(14) \\ C(28)-N(2)-M o(1) & 125.53(10) \\ C(32)-N(2)-M o(1) & 116.06(11) \\ O(1)-C(21)-C(26) & 119.20(12) \\ O(1)-C(21)-C(22) & 121.28(13) \\ C(26)-C(21)-C(22) & 119.49(12) \\ C(23)-C(22)-C(21) & 119.10(14) \\ C(23)-C(22)-C(28) & 118.35(13) \\ C(21)-C(22)-C(28) & 122.51(12) \\ C(24)-C(23)-C(22) & 122.02(14) \\ C(24)-C(23)-H(23) & 119.0 \\ C(22)-C(23)-H(23) & 119.0 \\ C(23)-C(24)-C(25) & 117.50(14) \\ C(23)-C(24)-C(27) & 120.74(15) \\ C(25)-C(24)-C(27) & 121.76(16) \\ C(26)-C(25)-C(24) & 123.01(15) \\ C(26)-C(25)-H(25) & 118.5 \\ C(24)-C(25)-H(25) & 118.5 \\ C(25)-C(26)-C(21) & 118.57(14) \\ C(25)-C(26)-C(33) & 119.33(13) \\ C(21)-C(26)-C(33) & 121.94(12) \\ C(24)-C(27)-H(27 A) & 109.5 \\ C(24)-C(27)-H(27 B) & 109.5 \\ H(27 A)-C(27)-H(27 B) & 109.5 \\ C(24)-C(27)-H(27 C) & 109.5 \\ H(27 A)-C(27)-H(27 C) & 109.5 \\ H(27 B)-C(27)-H(27 C) & 109.5 \\ N(2)-C(28)-C(29) & 119.60(14) \\ N(2)-C(28)-C(22) & 120.67(13) \\ C(29)-C(28)-C(22) & 119.72(14) \\ C(30)-C(29)-C(28) & 120.97(16) \\ C(30)-C(29)-H(29) & 119.5 \\ C(28)-C(29)-H(29) & 119.5 \\ C(29)-C(30)-C(31) & 118.71(16) \\ C(29)-C(30)-H(30) & 120.6 \\ C(31)-C(30)-H(30) & 120.6 \\ C(32)-C(31)-C(30) & 118.41(16) \\ C(32)-C(31)-H(31) & 120.8 \\ C(30)-C(31)-H(31) & 120.8 \\ N(2)-C(32)-C(31) & 123.83(16) \\ & \end{array}$

\begin{tabular}{|c|c|}
\hline$N(2)-C(32)-H(32)$ & 118.1 \\
\hline$C(31)-C(32)-H(32)$ & 118.1 \\
\hline $\mathrm{C}(21)-\mathrm{O}(1)-\mathrm{Mo}(1)$ & $127.55(9)$ \\
\hline$C(38)-C(33)-C(34)$ & $119.60(13)$ \\
\hline$C(38)-C(33)-C(26)$ & $118.33(14)$ \\
\hline$C(34)-C(33)-C(26)$ & $122.01(13)$ \\
\hline$C(35)-C(34)-C(33)$ & 118.99(14) \\
\hline$C(35)-C(34)-C(39)$ & $120.10(14)$ \\
\hline$C(33)-C(34)-C(39)$ & $120.88(13)$ \\
\hline$C(36)-C(35)-C(34)$ & $122.25(15)$ \\
\hline$C(36)-C(35)-H(35)$ & 118.9 \\
\hline$C(34)-C(35)-H(35)$ & 118.9 \\
\hline$C(37)-C(36)-C(35)$ & $117.57(14)$ \\
\hline$C(37)-C(36)-C(42)$ & $120.27(14)$ \\
\hline$C(35)-C(36)-C(42)$ & $122.12(15)$ \\
\hline$C(36)-C(37)-C(38)$ & $122.46(14)$ \\
\hline$C(36)-C(37)-H(37)$ & 118.8 \\
\hline$C(38)-C(37)-H(37)$ & 118.8 \\
\hline$C(37)-C(38)-C(33)$ & $119.11(15)$ \\
\hline$C(37)-C(38)-C(45)$ & $118.75(14)$ \\
\hline$C(33)-C(38)-C(45)$ & $122.10(14)$ \\
\hline$C(34)-C(39)-C(40)$ & $113.70(13)$ \\
\hline$C(34)-C(39)-C(41)$ & $110.78(14)$ \\
\hline$C(40)-C(39)-C(41)$ & $109.24(16)$ \\
\hline$C(34)-C(39)-H(39)$ & 107.6 \\
\hline$C(40)-C(39)-H(39)$ & 107.6 \\
\hline$C(41)-C(39)-H(39)$ & 107.6 \\
\hline $\mathrm{C}(39)-\mathrm{C}(40)-\mathrm{H}(40 \mathrm{~A})$ & 109.5 \\
\hline$C(39)-C(40)-H(40 B)$ & 109.5 \\
\hline$H(40 A)-C(40)-H(40 B)$ & 109.5 \\
\hline$C(39)-C(40)-H(40 C)$ & 109.5 \\
\hline $\mathrm{H}(40 \mathrm{~A})-\mathrm{C}(40)-\mathrm{H}(40 \mathrm{C})$ & 109.5 \\
\hline $\mathrm{H}(40 \mathrm{~B})-\mathrm{C}(40)-\mathrm{H}(40 \mathrm{C})$ & 109.5 \\
\hline$C(39)-C(41)-H(41 A)$ & 109.5 \\
\hline$C(39)-C(41)-H(41 B)$ & 109.5 \\
\hline $\mathrm{H}(41 \mathrm{~A})-\mathrm{C}(41)-\mathrm{H}(41 \mathrm{~B})$ & 109.5 \\
\hline $\mathrm{C}(39)-\mathrm{C}(41)-\mathrm{H}(41 \mathrm{C})$ & 109.5 \\
\hline $\mathrm{H}(41 \mathrm{~A})-\mathrm{C}(41)-\mathrm{H}(41 \mathrm{C})$ & 109.5 \\
\hline $\mathrm{H}(41 \mathrm{~B})-\mathrm{C}(41)-\mathrm{H}(41 \mathrm{C})$ & 109.5 \\
\hline$C(44)-C(42)-C(36)$ & $110.44(13)$ \\
\hline$C(44)-C(42)-C(43)$ & $110.80(16)$ \\
\hline$C(36)-C(42)-C(43)$ & $113.49(14)$ \\
\hline$C(44)-C(42)-H(42)$ & 107.3 \\
\hline$C(36)-C(42)-H(42)$ & 107.3 \\
\hline$C(43)-C(42)-H(42)$ & 107.3 \\
\hline$C(42)-C(43)-H(43 A)$ & 109.5 \\
\hline $\mathrm{C}(42)-\mathrm{C}(43)-\mathrm{H}(43 \mathrm{~B})$ & 109.5 \\
\hline $\mathrm{H}(43 \mathrm{~A})-\mathrm{C}(43)-\mathrm{H}(43 \mathrm{~B})$ & 109.5 \\
\hline $\mathrm{C}(42)-\mathrm{C}(43)-\mathrm{H}(43 \mathrm{C})$ & 109.5 \\
\hline$H(43 A)-C(43)-H(43 C)$ & 109.5 \\
\hline $\mathrm{H}(43 \mathrm{~B})-\mathrm{C}(43)-\mathrm{H}(43 \mathrm{C})$ & 109.5 \\
\hline $\mathrm{C}(42)-\mathrm{C}(44)-\mathrm{H}(44 \mathrm{~A})$ & 109.5 \\
\hline$C(42)-C(44)-H(44 B)$ & 109.5 \\
\hline
\end{tabular}




$\begin{array}{ll}H(44 A)-C(44)-H(44 B) & 109.5 \\ C(42)-C(44)-H(44 C) & 109.5 \\ H(44 A)-C(44)-H(44 C) & 109.5 \\ H(44 B)-C(44)-H(44 C) & 109.5 \\ C(38)-C(45)-C(46) & 112.30(14) \\ C(38)-C(45)-C(47) & 110.12(15) \\ C(46)-C(45)-C(47) & 110.68(16) \\ C(38)-C(45)-H(45) & 107.9 \\ C(46)-C(45)-H(45) & 107.9 \\ C(47)-C(45)-H(45) & 107.9 \\ C(45)-C(46)-H(46 A) & 109.5 \\ C(45)-C(46)-H(46 B) & 109.5 \\ H(46 A)-C(46)-H(46 B) & 109.5 \\ C(45)-C(46)-H(46 C) & 109.5 \\ H(46 A)-C(46)-H(46 C) & 109.5 \\ H(46 B)-C(46)-H(46 C) & 109.5 \\ C(45)-C(47)-H(47 A) & 109.5 \\ C(45)-C(47)-H(47 B) & 109.5 \\ H(47 A)-C(47)-H(47 B) & 109.5 \\ C(45)-C(47)-H(47 C) & 109.5 \\ H(47 A)-C(47)-H(47 C) & 109.5 \\ H(47 B)-C(47)-H(47 C) & 109.5 \\ C(2 S)-C(1 S)-H(1 S 1) & 109.5 \\ C(2 S)-C(1 S)-H(1 S 2) & 109.5 \\ H(1 S 1)-C(1 S)-H(1 S 2) & 109.5 \\ C(2 S)-C(1 S)-H(1 S 3) & 109.5 \\ H(1 S 1)-C(1 S)-H(1 S 3) & 109.5 \\ H(1 S 2)-C(1 S)-H(1 S 3) & 109.5 \\ C(3 S)-C(2 S)-C(1 S) & 113.4(4) \\ C(3 S)-C(2 S)-H(2 S 1) & 108.9 \\ C(1 S)-C(2 S)-H(2 S 1) & 108.9 \\ C(3 S)-C(2 S)-H(2 S 2) & 108.9 \\ C(1 S)-C(2 S)-H(2 S 2) & 108.9 \\ H(2 S 1)-C(2 S)-H(2 S 2) & 107.7 \\ C(2 S)-C(3 S)-C(4 S) & 115.9(4) \\ C(2 S)-C(3 S)-H(3 S 1) & 108.3 \\ C(4 S)-C(3 S)-H(3 S 1) & 108.3 \\ C(2 S)-C(3 S)-H(3 S 2) & 108.3 \\ C(4 S)-C(3 S)-H(3 S 2) & 108.3 \\ H(3 S 1)-C(3 S)-H(3 S 2) & 107.4 \\ C(3 S)-C(4 S)-C(5 S) & 114.7(4) \\ C(3 S)-C(4 S)-H(4 S 1) & 108.6 \\ & \\ & \end{array}$

\begin{tabular}{|c|c|}
\hline$C(5 S)-C(4 S)-H(4 S 1)$ & 108.6 \\
\hline $\mathrm{C}(3 \mathrm{~S})-\mathrm{C}(4 \mathrm{~S})-\mathrm{H}(4 \mathrm{~S} 2)$ & 108.6 \\
\hline $\mathrm{C}(5 \mathrm{~S})-\mathrm{C}(4 \mathrm{~S})-\mathrm{H}(4 \mathrm{~S} 2)$ & 108.6 \\
\hline $\mathrm{H}(4 \mathrm{~S} 1)-\mathrm{C}(4 \mathrm{~S})-\mathrm{H}(4 \mathrm{~S} 2)$ & 107.6 \\
\hline$C(4 S)-C(5 S)-H(5 S 1)$ & 109.5 \\
\hline$C(4 S)-C(5 S)-H(5 S 2)$ & 109.5 \\
\hline$H(5 S 1)-C(5 S)-H(5 S 2)$ & 109.5 \\
\hline $\mathrm{C}(4 \mathrm{~S})-\mathrm{C}(5 \mathrm{~S})-\mathrm{H}(5 \mathrm{~S} 3)$ & 109.5 \\
\hline$H(5 S 1)-C(5 S)-H(5 S 3)$ & 109.5 \\
\hline $\mathrm{H}(5 \mathrm{~S} 2)-\mathrm{C}(5 \mathrm{~S})-\mathrm{H}(5 \mathrm{~S} 3)$ & 109.5 \\
\hline $\mathrm{C}(2 \mathrm{~T})-\mathrm{C}(1 \mathrm{~T})-\mathrm{H}(1 \mathrm{~T} 1)$ & 109.5 \\
\hline $\mathrm{C}(2 \mathrm{~T})-\mathrm{C}(1 \mathrm{~T})-\mathrm{H}(1 \mathrm{~T} 2)$ & 109.5 \\
\hline $\mathrm{H}(1 \mathrm{~T} 1)-\mathrm{C}(1 \mathrm{~T})-\mathrm{H}(1 \mathrm{~T} 2)$ & 109.5 \\
\hline $\mathrm{C}(2 \mathrm{~T})-\mathrm{C}(1 \mathrm{~T})-\mathrm{H}(1 \mathrm{~T} 3)$ & 109.5 \\
\hline $\mathrm{H}(1 \mathrm{~T} 1)-\mathrm{C}(1 \mathrm{~T})-\mathrm{H}(1 \mathrm{~T} 3)$ & 109.5 \\
\hline $\mathrm{H}(1 \mathrm{~T} 2)-\mathrm{C}(1 \mathrm{~T})-\mathrm{H}(1 \mathrm{~T} 3)$ & 109.5 \\
\hline$C(3 T)-C(2 T)-C(1 T)$ & $113.3(13)$ \\
\hline $\mathrm{C}(3 \mathrm{~T})-\mathrm{C}(2 \mathrm{~T})-\mathrm{H}(2 \mathrm{~T} 1)$ & 108.9 \\
\hline $\mathrm{C}(1 \mathrm{~T})-\mathrm{C}(2 \mathrm{~T})-\mathrm{H}(2 \mathrm{~T} 1)$ & 108.9 \\
\hline $\mathrm{C}(3 \mathrm{~T})-\mathrm{C}(2 \mathrm{~T})-\mathrm{H}(2 \mathrm{~T} 2)$ & 108.9 \\
\hline $\mathrm{C}(1 \mathrm{~T})-\mathrm{C}(2 \mathrm{~T})-\mathrm{H}(2 \mathrm{~T} 2)$ & 108.9 \\
\hline $\mathrm{H}(2 \mathrm{~T} 1)-\mathrm{C}(2 \mathrm{~T})-\mathrm{H}(2 \mathrm{~T} 2)$ & 107.7 \\
\hline$C(2 T)-C(3 T)-C(4 T)$ & $114.6(13)$ \\
\hline $\mathrm{C}(2 \mathrm{~T})-\mathrm{C}(3 \mathrm{~T})-\mathrm{H}(3 \mathrm{~T} 1)$ & 108.6 \\
\hline $\mathrm{C}(4 \mathrm{~T})-\mathrm{C}(3 \mathrm{~T})-\mathrm{H}(3 \mathrm{~T} 1)$ & 108.6 \\
\hline $\mathrm{C}(2 \mathrm{~T})-\mathrm{C}(3 \mathrm{~T})-\mathrm{H}(3 \mathrm{~T} 2)$ & 108.6 \\
\hline $\mathrm{C}(4 \mathrm{~T})-\mathrm{C}(3 \mathrm{~T})-\mathrm{H}(3 \mathrm{~T} 2)$ & 108.6 \\
\hline $\mathrm{H}(3 \mathrm{~T} 1)-\mathrm{C}(3 \mathrm{~T})-\mathrm{H}(3 \mathrm{~T} 2)$ & 107.6 \\
\hline$C(3 T)-C(4 T)-C(5 T)$ & $113.8(12)$ \\
\hline $\mathrm{C}(3 \mathrm{~T})-\mathrm{C}(4 \mathrm{~T})-\mathrm{H}(4 \mathrm{~T} 1)$ & 108.8 \\
\hline $\mathrm{C}(5 \mathrm{~T})-\mathrm{C}(4 \mathrm{~T})-\mathrm{H}(4 \mathrm{~T} 1)$ & 108.8 \\
\hline $\mathrm{C}(3 \mathrm{~T})-\mathrm{C}(4 \mathrm{~T})-\mathrm{H}(4 \mathrm{~T} 2)$ & 108.8 \\
\hline $\mathrm{C}(5 \mathrm{~T})-\mathrm{C}(4 \mathrm{~T})-\mathrm{H}(4 \mathrm{~T} 2)$ & 108.8 \\
\hline $\mathrm{H}(4 \mathrm{~T} 1)-\mathrm{C}(4 \mathrm{~T})-\mathrm{H}(4 \mathrm{~T} 2)$ & 107.7 \\
\hline $\mathrm{C}(4 \mathrm{~T})-\mathrm{C}(5 \mathrm{~T})-\mathrm{H}(5 \mathrm{~T} 1)$ & 109.5 \\
\hline $\mathrm{C}(4 \mathrm{~T})-\mathrm{C}(5 \mathrm{~T})-\mathrm{H}(5 \mathrm{~T} 2)$ & 109.5 \\
\hline $\mathrm{H}(5 \mathrm{~T} 1)-\mathrm{C}(5 \mathrm{~T})-\mathrm{H}(5 \mathrm{~T} 2)$ & 109.5 \\
\hline $\mathrm{C}(4 \mathrm{~T})-\mathrm{C}(5 \mathrm{~T})-\mathrm{H}(5 \mathrm{~T} 3)$ & 109.5 \\
\hline $\mathrm{H}(5 \mathrm{~T} 1)-\mathrm{C}(5 \mathrm{~T})-\mathrm{H}(5 \mathrm{~T} 3)$ & 109.5 \\
\hline $\mathrm{H}(5 \mathrm{~T} 2)-\mathrm{C}(5 \mathrm{~T})-\mathrm{H}(5 \mathrm{~T} 3)$ & 109.5 \\
\hline
\end{tabular}


Table S9. Crystal data and structure refinement for X16064.

\begin{tabular}{|c|c|c|}
\hline Identification code & \multicolumn{2}{|l|}{ X16064 } \\
\hline Empirical formula & \multicolumn{2}{|c|}{$\mathrm{C}_{50} \mathrm{H}_{73} \mathrm{~F}_{3} \mathrm{Mo} \mathrm{N}_{2} \mathrm{O}_{6} \mathrm{~S}$} \\
\hline Formula weight & \multicolumn{2}{|l|}{983.10} \\
\hline Temperature & \multicolumn{2}{|l|}{$100(2) \mathrm{K}$} \\
\hline Wavelength & \multicolumn{2}{|l|}{$0.71073 \AA$} \\
\hline Crystal system & \multicolumn{2}{|l|}{ Triclinic } \\
\hline Space group & \multicolumn{2}{|l|}{$\mathrm{P} \overline{1}$} \\
\hline \multirow[t]{3}{*}{ Unit cell dimensions } & $a=11.0888(10) \AA$ & ? $=80.3137(18)^{\circ}$ \\
\hline & $b=14.4032(13) \AA$ & ? $=84.3917(19)^{\circ}$ \\
\hline & $c=16.6215(15) \AA$ & ? $=88.979(2)^{\circ}$ \\
\hline Volume & \multicolumn{2}{|l|}{$2604.3(4) \AA^{3}$} \\
\hline$Z$ & \multicolumn{2}{|l|}{2} \\
\hline Density (calculated) & \multicolumn{2}{|l|}{$1.254 \mathrm{Mg} / \mathrm{m}^{3}$} \\
\hline Absorption coefficient & \multicolumn{2}{|l|}{$0.349 \mathrm{~mm}^{-1}$} \\
\hline$F(000)$ & \multicolumn{2}{|l|}{1040} \\
\hline Crystal size & \multicolumn{2}{|c|}{$0.180 \times 0.140 \times 0.100 \mathrm{~mm}^{3}$} \\
\hline Theta range for data collection & \multicolumn{2}{|l|}{1.434 to $30.508^{\circ}$. } \\
\hline Index ranges & \multicolumn{2}{|c|}{$-15<=h<=15,-20<=k<=19,-23<=k<=23$} \\
\hline Reflections collected & \multicolumn{2}{|l|}{78095} \\
\hline Independent reflections & \multicolumn{2}{|c|}{$15864\left[R_{\text {int }}=0.0365\right]$} \\
\hline Completeness to theta $=25.242^{\circ}$ & \multicolumn{2}{|l|}{$100.0 \%$} \\
\hline Absorption correction & \multicolumn{2}{|c|}{ Semi-empirical from equivalents } \\
\hline Refinement method & \multicolumn{2}{|c|}{ Full-matrix least-squares on $F^{2}$} \\
\hline Data / restraints / parameters & \multicolumn{2}{|c|}{15864 / 2685 / 1000} \\
\hline Goodness-of-fit on $F^{2}$ & \multicolumn{2}{|l|}{1.050} \\
\hline Final $R$ indices $[/>2 \sigma(I)]$ & \multicolumn{2}{|c|}{$R 1=0.0493, w R 2=0.1257$} \\
\hline$R$ indices (all data) & \multicolumn{2}{|c|}{$R 1=0.0588, w R 2=0.1327$} \\
\hline Largest diff. peak and hole & \multicolumn{2}{|c|}{2.311 and -1.362 e. $\AA^{-3}$} \\
\hline Largest diff. peak and hole & \multicolumn{2}{|c|}{1.179 and -0.854 e. $\AA^{-3}$} \\
\hline
\end{tabular}


Table S10. Bond lengths $[\AA ̊]$ and angles $\left[{ }^{\circ}\right]$ for $\mathbf{X 1 6 0 6 4}$.

\begin{tabular}{|c|c|c|c|}
\hline$C(1)-C(2)$ & $1.397(4)$ & $C(24)-C(25)$ & $1.409(5)$ \\
\hline$C(1)-M o(1)$ & $2.200(2)$ & $C(24)-C(29)$ & $1.521(6)$ \\
\hline$C(1)-H(1 A)$ & $0.947(16)$ & $C(25)-C(26)$ & $1.386(5)$ \\
\hline$C(1)-H(1 B)$ & $0.951(16)$ & $\mathrm{C}(25)-\mathrm{H}(25)$ & 0.9500 \\
\hline $\mathrm{C}(2)-\mathrm{Mo}(1)$ & $2.193(2)$ & $C(26)-C(27)$ & $1.388(6)$ \\
\hline$C(2)-H(2 A)$ & $0.962(16)$ & $C(26)-C(32)$ & $1.526(4)$ \\
\hline$C(2)-H(2 B)$ & $0.969(16)$ & $C(27)-C(28)$ & $1.397(5)$ \\
\hline $\mathrm{Mo}(1)-\mathrm{N}(2)$ & $1.7658(18)$ & $C(27)-H(27)$ & 0.9500 \\
\hline $\mathrm{Mo}(1)-\mathrm{O}(1)$ & $1.9987(14)$ & $C(28)-C(35)$ & $1.523(6)$ \\
\hline $\mathrm{Mo}(1)-\mathrm{N}(1)$ & $2.1871(18)$ & $C(29)-C(31)$ & $1.529(5)$ \\
\hline $\mathrm{Mo}(1)-\mathrm{O}(2)$ & $2.2050(16)$ & $C(29)-C(30)$ & $1.530(5)$ \\
\hline $\mathrm{Mo}(1)-\mathrm{O}(5)$ & $2.2171(16)$ & $\mathrm{C}(29)-\mathrm{H}(29)$ & 1.0000 \\
\hline $\mathrm{O}(2)-\mathrm{S}(1)$ & $1.4301(19)$ & $C(30)-H(30 A)$ & 0.9800 \\
\hline$O(2)-S(1 A)$ & $1.5020(19)$ & $\mathrm{C}(30)-\mathrm{H}(30 \mathrm{~B})$ & 0.9800 \\
\hline$S(1)-O(4)$ & $1.429(4)$ & $C(30)-H(30 C)$ & 0.9800 \\
\hline $\mathrm{S}(1)-\mathrm{O}(3)$ & $1.449(9)$ & $C(31)-H(31 A)$ & 0.9800 \\
\hline$S(1)-C(3)$ & $1.815(5)$ & $\mathrm{C}(31)-\mathrm{H}(31 \mathrm{~B})$ & 0.9800 \\
\hline$C(3)-F(2)$ & $1.307(8)$ & $\mathrm{C}(31)-\mathrm{H}(31 \mathrm{C})$ & 0.9800 \\
\hline$C(3)-F(3)$ & $1.320(7)$ & $C(32)-C(34)$ & $1.514(5)$ \\
\hline$C(3)-F(1)$ & $1.337(8)$ & $C(32)-C(33)$ & $1.526(6)$ \\
\hline$S(1 A)-O(4 A)$ & $1.416(4)$ & $C(32)-H(32)$ & 1.0000 \\
\hline$S(1 A)-O(3 A)$ & $1.429(9)$ & $C(33)-H(33 A)$ & 0.9800 \\
\hline$S(1 A)-C(3 A)$ & $1.819(5)$ & $\mathrm{C}(33)-\mathrm{H}(33 \mathrm{~B})$ & 0.9800 \\
\hline$C(3 A)-F(1 A)$ & $1.287(9)$ & $\mathrm{C}(33)-\mathrm{H}(33 \mathrm{C})$ & 0.9800 \\
\hline$C(3 A)-F(2 A)$ & $1.297(9)$ & $C(34)-H(34 A)$ & 0.9800 \\
\hline$C(3 A)-F(3 A)$ & $1.318(7)$ & $\mathrm{C}(34)-\mathrm{H}(34 \mathrm{~B})$ & 0.9800 \\
\hline$O(1)-C(11)$ & $1.329(2)$ & $\mathrm{C}(34)-\mathrm{H}(34 \mathrm{C})$ & 0.9800 \\
\hline$C(11)-C(16)$ & $1.410(2)$ & $C(35)-C(37)$ & $1.531(5)$ \\
\hline$C(11)-C(12)$ & $1.414(3)$ & $C(35)-C(36)$ & $1.532(5)$ \\
\hline$C(12)-C(13)$ & $1.408(3)$ & $\mathrm{C}(35)-\mathrm{H}(35)$ & 1.0000 \\
\hline$C(12)-C(18)$ & $1.483(3)$ & $C(36)-H(36 A)$ & 0.9800 \\
\hline$N(1)-C(18)$ & $1.358(3)$ & $C(36)-H(36 B)$ & 0.9800 \\
\hline$N(1)-C(22)$ & $1.360(2)$ & $\mathrm{C}(36)-\mathrm{H}(36 \mathrm{C})$ & 0.9800 \\
\hline$C(18)-C(19)$ & $1.403(3)$ & $\mathrm{C}(37)-\mathrm{H}(37 \mathrm{~A})$ & 0.9800 \\
\hline$C(19)-C(20)$ & $1.377(3)$ & $\mathrm{C}(37)-\mathrm{H}(37 \mathrm{~B})$ & 0.9800 \\
\hline $\mathrm{C}(19)-\mathrm{H}(19)$ & 0.9500 & $\mathrm{C}(37)-\mathrm{H}(37 \mathrm{C})$ & 0.9800 \\
\hline$C(20)-C(21)$ & $1.386(3)$ & $C(23 A)-C(24 A)$ & $1.391(7)$ \\
\hline $\mathrm{C}(20)-\mathrm{H}(20)$ & 0.9500 & $C(23 A)-C(28 A)$ & $1.405(7)$ \\
\hline$C(21)-C(22)$ & $1.369(3)$ & $C(24 A)-C(25 A)$ & $1.401(7)$ \\
\hline $\mathrm{C}(21)-\mathrm{H}(21)$ & 0.9500 & $C(24 A)-C(29 A)$ & $1.518(7)$ \\
\hline $\mathrm{C}(22)-\mathrm{H}(22)$ & 0.9500 & $C(25 A)-C(26 A)$ & $1.385(8)$ \\
\hline$C(13)-C(14)$ & $1.382(3)$ & $C(25 A)-H(25 A)$ & 0.9500 \\
\hline $\mathrm{C}(13)-\mathrm{H}(13)$ & 0.9500 & $C(26 A)-C(27 A)$ & $1.380(8)$ \\
\hline$C(14)-C(15)$ & $1.394(3)$ & $C(26 A)-C(32 A)$ & $1.523(6)$ \\
\hline$C(14)-C(17)$ & $1.506(3)$ & $C(27 A)-C(28 A)$ & $1.390(7)$ \\
\hline$C(15)-C(16)$ & $1.391(3)$ & $C(27 A)-H(27 A)$ & 0.9500 \\
\hline $\mathrm{C}(15)-\mathrm{H}(15)$ & 0.9500 & $C(28 \mathrm{~A})-C(35 \mathrm{~A})$ & $1.519(7)$ \\
\hline$C(16)-C(23 A)$ & $1.491(6)$ & $C(29 A)-C(31 A)$ & $1.523(7)$ \\
\hline$C(16)-C(23)$ & $1.510(4)$ & $C(29 A)-C(30 A)$ & $1.527(8)$ \\
\hline$C(23)-C(24)$ & $1.396(5)$ & $C(29 A)-H(29 A)$ & 1.0000 \\
\hline$C(23)-C(28)$ & $1.415(5)$ & $C(30 A)-H(30 D)$ & 0.9800 \\
\hline
\end{tabular}




\begin{tabular}{|c|c|c|c|}
\hline$C(30 A)-H(30 E)$ & 0.9800 & $C(51)-H(51 C)$ & 0.9800 \\
\hline$C(30 A)-H(30 F)$ & 0.9800 & $C(52)-H(52 A)$ & 0.9800 \\
\hline$C(31 A)-H(31 D)$ & 0.9800 & $C(52)-H(52 B)$ & 0.9800 \\
\hline$C(31 A)-H(31 E)$ & 0.9800 & $C(52)-H(52 C)$ & 0.9800 \\
\hline$C(31 A)-H(31 F)$ & 0.9800 & $C(41 A)-C(46 A)$ & $1.352(10)$ \\
\hline$C(32 A)-C(34 A)$ & $1.510(8)$ & $C(41 A)-C(42 A)$ & $1.385(10)$ \\
\hline$C(32 A)-C(33 A)$ & $1.526(9)$ & $C(42 A)-C(43 A)$ & $1.396(8)$ \\
\hline$C(32 A)-H(32 A)$ & 1.0000 & $C(42 A)-C(47 A)$ & $1.508(9)$ \\
\hline$C(33 A)-H(33 D)$ & 0.9800 & $C(43 A)-C(44 A)$ & $1.376(8)$ \\
\hline $\mathrm{C}(33 \mathrm{~A})-\mathrm{H}(33 \mathrm{E})$ & 0.9800 & $\mathrm{C}(43 \mathrm{~A})-\mathrm{H}(43 \mathrm{~A})$ & 0.9500 \\
\hline$C(33 A)-H(33 F)$ & 0.9800 & $C(44 A)-C(45 A)$ & $1.368(9)$ \\
\hline$C(34 A)-H(34 D)$ & 0.9800 & $C(44 A)-H(44 A)$ & 0.9500 \\
\hline$C(34 A)-H(34 E)$ & 0.9800 & $C(45 A)-C(46 A)$ & $1.410(10)$ \\
\hline $\mathrm{C}(34 \mathrm{~A})-\mathrm{H}(34 \mathrm{~F})$ & 0.9800 & $C(45 A)-H(45 A)$ & 0.9500 \\
\hline$C(35 A)-C(36 A)$ & $1.528(8)$ & $C(46 A)-C(50 A)$ & $1.489(10)$ \\
\hline$C(35 A)-C(37 A)$ & $1.533(7)$ & $C(47 A)-C(48 A)$ & $1.521(9)$ \\
\hline$C(35 A)-H(35 A)$ & 1.0000 & $C(47 A)-C(49 A)$ & $1.522(8)$ \\
\hline$C(36 A)-H(36 D)$ & 0.9800 & $C(47 A)-H(47 A)$ & 1.0000 \\
\hline$C(36 A)-H(36 E)$ & 0.9800 & $\mathrm{C}(48 \mathrm{~A})-\mathrm{H}(48 \mathrm{D})$ & 0.9800 \\
\hline$C(36 A)-H(36 F)$ & 0.9800 & $\mathrm{C}(48 \mathrm{~A})-\mathrm{H}(48 \mathrm{E})$ & 0.9800 \\
\hline$C(37 A)-H(37 D)$ & 0.9800 & $\mathrm{C}(48 \mathrm{~A})-\mathrm{H}(48 \mathrm{~F})$ & 0.9800 \\
\hline $\mathrm{C}(37 \mathrm{~A})-\mathrm{H}(37 \mathrm{E})$ & 0.9800 & $C(49 A)-H(49 D)$ & 0.9800 \\
\hline $\mathrm{C}(37 \mathrm{~A})-\mathrm{H}(37 \mathrm{~F})$ & 0.9800 & $\mathrm{C}(49 \mathrm{~A})-\mathrm{H}(49 \mathrm{E})$ & 0.9800 \\
\hline$C(17)-H(17 A)$ & 0.9800 & $\mathrm{C}(49 \mathrm{~A})-\mathrm{H}(49 \mathrm{~F})$ & 0.9800 \\
\hline$C(17)-H(17 B)$ & 0.9800 & $C(50 A)-C(52 A)$ & $1.497(10)$ \\
\hline$C(17)-H(17 C)$ & 0.9800 & $C(50 A)-C(51 A)$ & $1.522(10)$ \\
\hline$N(2)-C(41 A)$ & $1.385(9)$ & $\mathrm{C}(50 \mathrm{~A})-\mathrm{H}(50 \mathrm{~A})$ & 1.0000 \\
\hline$N(2)-C(41)$ & $1.408(7)$ & $C(51 A)-H(51 D)$ & 0.9800 \\
\hline$C(41)-C(42)$ & $1.441(9)$ & $\mathrm{C}(51 \mathrm{~A})-\mathrm{H}(51 \mathrm{E})$ & 0.9800 \\
\hline$C(41)-C(46)$ & $1.452(9)$ & $\mathrm{C}(51 \mathrm{~A})-\mathrm{H}(51 \mathrm{~F})$ & 0.9800 \\
\hline$C(42)-C(43)$ & $1.413(8)$ & $C(52 A)-H(52 D)$ & 0.9800 \\
\hline$C(42)-C(47)$ & $1.527(8)$ & $C(52 A)-H(52 E)$ & 0.9800 \\
\hline$C(43)-C(44)$ & $1.368(8)$ & $\mathrm{C}(52 \mathrm{~A})-\mathrm{H}(52 \mathrm{~F})$ & 0.9800 \\
\hline $\mathrm{C}(43)-\mathrm{H}(43)$ & 0.9500 & $C(62)-C(61)$ & $1.509(4)$ \\
\hline$C(44)-C(45)$ & $1.376(9)$ & $\mathrm{C}(62)-\mathrm{H}(62 \mathrm{~A})$ & 0.9800 \\
\hline $\mathrm{C}(44)-\mathrm{H}(44)$ & 0.9500 & $\mathrm{C}(62)-\mathrm{H}(62 \mathrm{~B})$ & 0.9800 \\
\hline$C(45)-C(46)$ & $1.450(9)$ & $\mathrm{C}(62)-\mathrm{H}(62 \mathrm{C})$ & 0.9800 \\
\hline $\mathrm{C}(45)-\mathrm{H}(45)$ & 0.9500 & $C(61)-O(5)$ & $1.450(3)$ \\
\hline$C(46)-C(50)$ & $1.541(9)$ & $C(61)-H(61 A)$ & 0.9900 \\
\hline$C(47)-C(48)$ & $1.525(9)$ & $C(61)-H(61 B)$ & 0.9900 \\
\hline$C(47)-C(49)$ & $1.537(9)$ & $\mathrm{O}(5)-\mathrm{C}(63)$ & $1.433(8)$ \\
\hline $\mathrm{C}(47)-\mathrm{H}(47)$ & 1.0000 & $O(5)-C(63 A)$ & $1.491(10)$ \\
\hline $\mathrm{C}(48)-\mathrm{H}(48 \mathrm{~A})$ & 0.9800 & $C(63)-C(64)$ & $1.489(10)$ \\
\hline $\mathrm{C}(48)-\mathrm{H}(48 \mathrm{~B})$ & 0.9800 & $C(63)-H(63 A)$ & 0.9900 \\
\hline $\mathrm{C}(48)-\mathrm{H}(48 \mathrm{C})$ & 0.9800 & $\mathrm{C}(63)-\mathrm{H}(63 \mathrm{~B})$ & 0.9900 \\
\hline$C(49)-H(49 A)$ & 0.9800 & $C(64)-H(64 A)$ & 0.9800 \\
\hline$C(49)-H(49 B)$ & 0.9800 & $\mathrm{C}(64)-\mathrm{H}(64 \mathrm{~B})$ & 0.9800 \\
\hline $\mathrm{C}(49)-\mathrm{H}(49 \mathrm{C})$ & 0.9800 & $\mathrm{C}(64)-\mathrm{H}(64 \mathrm{C})$ & 0.9800 \\
\hline$C(50)-C(52)$ & $1.541(9)$ & $C(63 A)-C(64 A)$ & $1.493(13)$ \\
\hline$C(50)-C(51)$ & $1.551(8)$ & $\mathrm{C}(63 \mathrm{~A})-\mathrm{H}(63 \mathrm{C})$ & 0.9900 \\
\hline$C(50)-H(50)$ & 1.0000 & $C(63 A)-H(63 D)$ & 0.9900 \\
\hline$C(51)-H(51 A)$ & 0.9800 & $C(64 A)-H(64 D)$ & 0.9800 \\
\hline$C(51)-H(51 B)$ & 0.9800 & $C(64 A)-H(64 E)$ & 0.9800 \\
\hline
\end{tabular}




\begin{tabular}{|c|c|c|c|}
\hline$C(64 A)-H(64 F)$ & 0.9800 & $C(1)-C(2)-H(2 B)$ & $123.1(17)$ \\
\hline$C(2 S)-C(1 S)$ & $1.473(9)$ & $\mathrm{Mo}(1)-\mathrm{C}(2)-\mathrm{H}(2 \mathrm{~B})$ & 109.0(15) \\
\hline $\mathrm{C}(2 \mathrm{~S})-\mathrm{H}(2 \mathrm{~S} 1)$ & 0.9800 & $H(2 A)-C(2)-H(2 B)$ & $111(2)$ \\
\hline $\mathrm{C}(2 \mathrm{~S})-\mathrm{H}(2 \mathrm{~S} 2)$ & 0.9800 & $N(2)-M o(1)-O(1)$ & $177.12(8)$ \\
\hline $\mathrm{C}(2 \mathrm{~S})-\mathrm{H}(2 \mathrm{~S} 3)$ & 0.9800 & $N(2)-M o(1)-N(1)$ & $94.52(7)$ \\
\hline$C(1 S)-O(1 S)$ & $1.513(7)$ & $\mathrm{O}(1)-\mathrm{Mo}(1)-\mathrm{N}(1)$ & $83.20(6)$ \\
\hline $\mathrm{C}(1 \mathrm{~S})-\mathrm{H}(1 \mathrm{~S} 1)$ & 0.9900 & $\mathrm{~N}(2)-\mathrm{Mo}(1)-\mathrm{C}(2)$ & $95.56(9)$ \\
\hline $\mathrm{C}(1 \mathrm{~S})-\mathrm{H}(1 \mathrm{~S} 2)$ & 0.9900 & $\mathrm{O}(1)-\mathrm{Mo}(1)-\mathrm{C}(2)$ & $87.03(8)$ \\
\hline$O(1 S)-C(3 S)$ & $1.511(7)$ & $\mathrm{N}(1)-\mathrm{Mo}(1)-\mathrm{C}(2)$ & $115.78(8)$ \\
\hline$C(3 S)-C(4 S)$ & $1.480(9)$ & $\mathrm{N}(2)-\mathrm{Mo}(1)-\mathrm{C}(1)$ & 94.99(9) \\
\hline$C(3 S)-H(3 S 1)$ & 0.9900 & $\mathrm{O}(1)-\mathrm{Mo}(1)-\mathrm{C}(1)$ & $86.31(7)$ \\
\hline $\mathrm{C}(3 \mathrm{~S})-\mathrm{H}(3 \mathrm{~S} 2)$ & 0.9900 & $N(1)-M o(1)-C(1)$ & $78.93(8)$ \\
\hline$C(4 S)-H(4 S 1)$ & 0.9800 & $C(2)-M o(1)-C(1)$ & $37.07(9)$ \\
\hline $\mathrm{C}(4 \mathrm{~S})-\mathrm{H}(4 \mathrm{~S} 2)$ & 0.9800 & $\mathrm{~N}(2)-\mathrm{Mo}(1)-\mathrm{O}(2)$ & $94.83(8)$ \\
\hline $\mathrm{C}(4 \mathrm{~S})-\mathrm{H}(4 \mathrm{~S} 3)$ & 0.9800 & $\mathrm{O}(1)-\mathrm{Mo}(1)-\mathrm{O}(2)$ & $83.15(6)$ \\
\hline$C(2 T)-C(1 T)$ & $1.485(10)$ & $\mathrm{N}(1)-\mathrm{Mo}(1)-\mathrm{O}(2)$ & $82.50(6)$ \\
\hline $\mathrm{C}(2 \mathrm{~T})-\mathrm{H}(2 \mathrm{~T} 1)$ & 0.9800 & $\mathrm{C}(2)-\mathrm{Mo}(1)-\mathrm{O}(2)$ & 158.09(8) \\
\hline $\mathrm{C}(2 \mathrm{~T})-\mathrm{H}(2 \mathrm{~T} 2)$ & 0.9800 & $\mathrm{C}(1)-\mathrm{Mo}(1)-\mathrm{O}(2)$ & $159.60(8)$ \\
\hline$C(2 \mathrm{~T})-\mathrm{H}(2 \mathrm{~T} 3)$ & 0.9800 & $\mathrm{~N}(2)-\mathrm{Mo}(1)-\mathrm{O}(5)$ & $95.90(8)$ \\
\hline$C(1 T)-O(1 T)$ & $1.486(8)$ & $\mathrm{O}(1)-\mathrm{Mo}(1)-\mathrm{O}(5)$ & $85.75(6)$ \\
\hline$C(1 T)-H(1 T 1)$ & 0.9900 & $\mathrm{~N}(1)-\mathrm{Mo}(1)-\mathrm{O}(5)$ & $159.66(7)$ \\
\hline$C(1 T)-H(1 T 2)$ & 0.9900 & $\mathrm{C}(2)-\mathrm{Mo}(1)-\mathrm{O}(5)$ & $80.52(9)$ \\
\hline $\mathrm{O}(1 \mathrm{~T})-\mathrm{C}(3 \mathrm{~T})$ & $1.524(8)$ & $\mathrm{C}(1)-\mathrm{Mo}(1)-\mathrm{O}(5)$ & $117.38(8)$ \\
\hline$C(3 T)-C(4 T)$ & $1.478(10)$ & $\mathrm{O}(2)-\mathrm{Mo}(1)-\mathrm{O}(5)$ & $79.26(6)$ \\
\hline $\mathrm{C}(3 \mathrm{~T})-\mathrm{H}(3 \mathrm{~T} 1)$ & 0.9900 & $\mathrm{~S}(1)-\mathrm{O}(2)-\mathrm{Mo}(1)$ & $138.57(11)$ \\
\hline $\mathrm{C}(3 \mathrm{~T})-\mathrm{H}(3 \mathrm{~T} 2)$ & 0.9900 & $\mathrm{~S}(1 \mathrm{~A})-\mathrm{O}(2)-\mathrm{Mo}(1)$ & $134.51(10)$ \\
\hline $\mathrm{C}(4 \mathrm{~T})-\mathrm{H}(4 \mathrm{~T} 1)$ & 0.9800 & $O(4)-S(1)-O(2)$ & $112.60(18)$ \\
\hline $\mathrm{C}(4 \mathrm{~T})-\mathrm{H}(4 \mathrm{~T} 2)$ & 0.9800 & $O(4)-S(1)-O(3)$ & $118.7(6)$ \\
\hline $\mathrm{C}(4 \mathrm{~T})-\mathrm{H}(4 \mathrm{~T} 3)$ & 0.9800 & $O(2)-S(1)-O(3)$ & $113.4(5)$ \\
\hline$C(2 U)-C(1 U)$ & $1.477(10)$ & $O(4)-S(1)-C(3)$ & $104.4(3)$ \\
\hline$C(2 U)-H(2 U 1)$ & 0.9800 & $\mathrm{O}(2)-\mathrm{S}(1)-\mathrm{C}(3)$ & $99.7(2)$ \\
\hline $\mathrm{C}(2 \mathrm{U})-\mathrm{H}(2 \mathrm{U} 2)$ & 0.9800 & $\mathrm{O}(3)-\mathrm{S}(1)-\mathrm{C}(3)$ & $105.2(6)$ \\
\hline$C(2 U)-H(2 U 3)$ & 0.9800 & $F(2)-C(3)-F(3)$ & $103.8(7)$ \\
\hline$C(1 U)-O(1 U)$ & $1.498(9)$ & $F(2)-C(3)-F(1)$ & 109.0(8) \\
\hline$C(1 \cup)-H(1 \cup 1)$ & 0.9900 & $F(3)-C(3)-F(1)$ & 105.9(5) \\
\hline$C(1 U)-H(1 \cup 2)$ & 0.9900 & $F(2)-C(3)-S(1)$ & $114.7(8)$ \\
\hline$O(1 U)-C(3 U)$ & $1.503(9)$ & $F(3)-C(3)-S(1)$ & $111.0(4)$ \\
\hline$C(3 U)-C(4 U)$ & $1.484(10)$ & $F(1)-C(3)-S(1)$ & $111.8(6)$ \\
\hline$C(3 U)-H(3 U 1)$ & 0.9900 & $O(4 A)-S(1 A)-O(3 A)$ & $118.9(7)$ \\
\hline $\mathrm{C}(3 \mathrm{U})-\mathrm{H}(3 \mathrm{U} 2)$ & 0.9900 & $O(4 A)-S(1 A)-O(2)$ & $110.8(2)$ \\
\hline$C(4 U)-H(4 U 1)$ & 0.9800 & $O(3 A)-S(1 A)-O(2)$ & $117.2(6)$ \\
\hline $\mathrm{C}(4 \mathrm{U})-\mathrm{H}(4 \mathrm{U} 2)$ & 0.9800 & $O(4 A)-S(1 A)-C(3 A)$ & $103.7(3)$ \\
\hline \multirow[t]{2}{*}{$\mathrm{C}(4 \mathrm{U})-\mathrm{H}(4 \mathrm{U})$} & 0.9800 & $O(3 A)-S(1 A)-C(3 A)$ & $100.9(7)$ \\
\hline & & $O(2)-S(1 A)-C(3 A)$ & $102.0(2)$ \\
\hline$C(2)-C(1)-M o(1)$ & $71.18(14)$ & $F(1 A)-C(3 A)-F(2 A)$ & 111.2(9) \\
\hline$C(2)-C(1)-H(1 A)$ & $121.0(17)$ & $F(1 A)-C(3 A)-F(3 A)$ & $102.7(8)$ \\
\hline$M o(1)-C(1)-H(1 A)$ & $107.2(15)$ & $F(2 A)-C(3 A)-F(3 A)$ & $105.8(6)$ \\
\hline$C(2)-C(1)-H(1 B)$ & $122.5(17)$ & $F(1 A)-C(3 A)-S(1 A)$ & $111.9(7)$ \\
\hline $\mathrm{Mo}(1)-C(1)-H(1 B)$ & $111.2(15)$ & $F(2 A)-C(3 A)-S(1 A)$ & $113.3(6)$ \\
\hline$H(1 A)-C(1)-H(1 B)$ & $113(2)$ & $F(3 A)-C(3 A)-S(1 A)$ & $111.4(4)$ \\
\hline$C(1)-C(2)-M o(1)$ & $71.75(13)$ & $\mathrm{C}(11)-\mathrm{O}(1)-\mathrm{Mo}(1)$ & $132.39(12)$ \\
\hline$C(1)-C(2)-H(2 A)$ & $123.2(17)$ & $O(1)-C(11)-C(16)$ & $118.54(18)$ \\
\hline$M o(1)-C(2)-H(2 A)$ & $108.9(15)$ & $O(1)-C(11)-C(12)$ & $122.09(16)$ \\
\hline
\end{tabular}




\begin{tabular}{|c|c|}
\hline$C(16)-C(11)-C(12)$ & $119.37(18)$ \\
\hline$C(13)-C(12)-C(11)$ & $118.59(17)$ \\
\hline$C(13)-C(12)-C(18)$ & $118.30(18)$ \\
\hline$C(11)-C(12)-C(18)$ & $122.98(18)$ \\
\hline$C(18)-N(1)-C(22)$ & $118.38(18)$ \\
\hline $\mathrm{C}(18)-\mathrm{N}(1)-\mathrm{Mo}(1)$ & $126.64(13)$ \\
\hline $\mathrm{C}(22)-\mathrm{N}(1)-\mathrm{Mo}(1)$ & $114.76(14)$ \\
\hline$N(1)-C(18)-C(19)$ & $119.35(17)$ \\
\hline$N(1)-C(18)-C(12)$ & $122.47(18)$ \\
\hline$C(19)-C(18)-C(12)$ & $118.17(18)$ \\
\hline$C(20)-C(19)-C(18)$ & $121.6(2)$ \\
\hline$C(20)-C(19)-H(19)$ & 119.2 \\
\hline$C(18)-C(19)-H(19)$ & 119.2 \\
\hline$C(19)-C(20)-C(21)$ & $118.3(2)$ \\
\hline$C(19)-C(20)-H(20)$ & 120.8 \\
\hline$C(21)-C(20)-H(20)$ & 120.8 \\
\hline$C(22)-C(21)-C(20)$ & $118.5(2)$ \\
\hline$C(22)-C(21)-H(21)$ & 120.8 \\
\hline$C(20)-C(21)-H(21)$ & 120.8 \\
\hline$N(1)-C(22)-C(21)$ & $123.9(2)$ \\
\hline $\mathrm{N}(1)-\mathrm{C}(22)-\mathrm{H}(22)$ & 118.1 \\
\hline$C(21)-C(22)-H(22)$ & 118.1 \\
\hline$C(14)-C(13)-C(12)$ & $122.66(19)$ \\
\hline$C(14)-C(13)-H(13)$ & 118.7 \\
\hline$C(12)-C(13)-H(13)$ & 118.7 \\
\hline$C(13)-C(14)-C(15)$ & $117.42(19)$ \\
\hline$C(13)-C(14)-C(17)$ & $121.5(2)$ \\
\hline$C(15)-C(14)-C(17)$ & $121.12(19)$ \\
\hline$C(16)-C(15)-C(14)$ & $122.56(18)$ \\
\hline$C(16)-C(15)-H(15)$ & 118.7 \\
\hline$C(14)-C(15)-H(15)$ & 118.7 \\
\hline$C(15)-C(16)-C(11)$ & $119.27(19)$ \\
\hline$C(15)-C(16)-C(23 A)$ & $115.9(8)$ \\
\hline$C(11)-C(16)-C(23 A)$ & $124.3(8)$ \\
\hline$C(15)-C(16)-C(23)$ & $118.3(5)$ \\
\hline$C(11)-C(16)-C(23)$ & $122.2(5)$ \\
\hline$C(24)-C(23)-C(28)$ & $118.7(4)$ \\
\hline$C(24)-C(23)-C(16)$ & $121.0(4)$ \\
\hline$C(28)-C(23)-C(16)$ & $120.0(4)$ \\
\hline$C(23)-C(24)-C(25)$ & $119.6(4)$ \\
\hline$C(23)-C(24)-C(29)$ & $121.0(3)$ \\
\hline$C(25)-C(24)-C(29)$ & $119.3(4)$ \\
\hline$C(26)-C(25)-C(24)$ & $121.8(4)$ \\
\hline$C(26)-C(25)-H(25)$ & 119.1 \\
\hline$C(24)-C(25)-H(25)$ & 119.1 \\
\hline$C(25)-C(26)-C(27)$ & $118.2(3)$ \\
\hline$C(25)-C(26)-C(32)$ & $120.5(4)$ \\
\hline$C(27)-C(26)-C(32)$ & $121.3(4)$ \\
\hline$C(26)-C(27)-C(28)$ & $121.5(4)$ \\
\hline$C(26)-C(27)-H(27)$ & 119.3 \\
\hline$C(28)-C(27)-H(27)$ & 119.3 \\
\hline$C(27)-C(28)-C(23)$ & $120.0(4)$ \\
\hline$C(27)-C(28)-C(35)$ & $119.1(4)$ \\
\hline
\end{tabular}

$\begin{array}{ll}C(23)-C(28)-C(35) & 120.7(4) \\ C(24)-C(29)-C(31) & 114.4(4) \\ C(24)-C(29)-C(30) & 110.1(4) \\ C(31)-C(29)-C(30) & 109.2(5) \\ C(24)-C(29)-H(29) & 107.6 \\ C(31)-C(29)-H(29) & 107.6 \\ C(30)-C(29)-H(29) & 107.6 \\ C(29)-C(30)-H(30 A) & 109.5 \\ C(29)-C(30)-H(30 B) & 109.5 \\ H(30 A)-C(30)-H(30 B) & 109.5 \\ C(29)-C(30)-H(30 C) & 109.5 \\ H(30 A)-C(30)-H(30 C) & 109.5 \\ H(30 B)-C(30)-H(30 C) & 109.5 \\ C(29)-C(31)-H(31 A) & 109.5 \\ C(29)-C(31)-H(31 B) & 109.5 \\ H(31 A)-C(31)-H(31 B) & 109.5 \\ C(29)-C(31)-H(31 C) & 109.5 \\ H(31 A)-C(31)-H(31 C) & 109.5 \\ H(31 B)-C(31)-H(31 C) & 109.5 \\ C(34)-C(32)-C(33) & 110.7(6) \\ C(34)-C(32)-C(26) & 113.2(4) \\ C(33)-C(32)-C(26) & 110.8(4) \\ C(34)-C(32)-H(32) & 107.3 \\ C(33)-C(32)-H(32) & 107.3 \\ C(26)-C(32)-H(32) & 107.3 \\ C(32)-C(33)-H(33 A) & 109.5 \\ C(32)-C(33)-H(33 B) & 109.5 \\ H(33 A)-C(33)-H(33 B) & 109.5 \\ C(32)-C(33)-H(33 C) & 109.5 \\ H(33 A)-C(33)-H(33 C) & 109.5 \\ H(33 B)-C(33)-H(33 C) & 109.5 \\ C(32)-C(34)-H(34 A) & 109.5 \\ C(32)-C(34)-H(34 B) & 109.5 \\ H(34 A)-C(34)-H(34 B) & 109.5 \\ C(32)-C(34)-H(34 C) & 109.5 \\ H(34 A)-C(34)-H(34 C) & 109.5 \\ H(34 B)-C(34)-H(34 C) & 109.5 \\ C(28)-C(35)-C(37) & 112.9(4) \\ C(28)-C(35)-C(36) & 110.7(4) \\ C(37)-C(35)-C(36) & 110.2(4) \\ C(28)-C(35)-H(35) & 107.6 \\ C(37)-C(35)-H(35) & 107.6 \\ C(36)-C(35)-H(35) & 107.6 \\ C(35)-C(36)-H(36 A) & 109.5 \\ C(35)-C(36)-H(36 B) & 109.5 \\ H(36 A)-C(36)-H(36 B) & 109.5 \\ C(35)-C(36)-H(36 C) & 109.5 \\ H(36 A)-C(36)-H(36 C) & 109.5 \\ H(36 B)-C(36)-H(36 C) & 109.5 \\ C(35)-C(37)-H(37 A) & 109.5 \\ C(35)-C(37)-H(37 B) & 109.5 \\ H(37 A)-C(37)-H(37 B) & 109.5 \\ C(35)-C(37)-H(37 C) & 109.5 \\ & \end{array}$




$\begin{array}{ll}H(37 A)-C(37)-H(37 C) & 109.5 \\ H(37 B)-C(37)-H(37 C) & 109.5 \\ C(24 A)-C(23 A)-C(28 A) & 121.3(5) \\ C(24 A)-C(23 A)-C(16) & 117.7(5) \\ C(28 A)-C(23 A)-C(16) & 120.3(5) \\ C(23 A)-C(24 A)-C(25 A) & 119.0(5) \\ C(23 A)-C(24 A)-C(29 A) & 120.7(5) \\ C(25 A)-C(24 A)-C(29 A) & 120.1(5) \\ C(26 A)-C(25 A)-C(24 A) & 120.4(5) \\ C(26 A)-C(25 A)-H(25 A) & 119.8 \\ C(24 A)-C(25 A)-H(25 A) & 119.8 \\ C(27 A)-C(26 A)-C(25 A) & 119.3(5) \\ C(27 A)-C(26 A)-C(32 A) & 120.9(6) \\ C(25 A)-C(26 A)-C(32 A) & 119.8(6) \\ C(26 A)-C(27 A)-C(28 A) & 122.3(5) \\ C(26 A)-C(27 A)-H(27 A) & 118.9 \\ C(28 A)-C(27 A)-H(27 A) & 118.9 \\ C(27 A)-C(28 A)-C(23 A) & 117.5(5) \\ C(27 A)-C(28 A)-C(35 A) & 120.1(5) \\ C(23 A)-C(28 A)-C(35 A) & 122.3(5) \\ C(24 A)-C(29 A)-C(31 A) & 114.4(6) \\ C(24 A)-C(29 A)-C(30 A) & 110.9(7) \\ C(31 A)-C(29 A)-C(30 A) & 110.3(8) \\ C(24 A)-C(29 A)-H(29 A) & 106.9 \\ C(31 A)-C(29 A)-H(29 A) & 106.9 \\ C(30 A)-C(29 A)-H(29 A) & 106.9 \\ C(29 A)-C(30 A)-H(30 D) & 109.5 \\ C(29 A)-C(30 A)-H(30 E) & 109.5 \\ H(30 D)-C(30 A)-H(30 E) & 109.5 \\ C(29 A)-C(30 A)-H(30 F) & 109.5 \\ H(30 D)-C(30 A)-H(30 F) & 109.5 \\ H(30 E)-C(30 A)-H(30 F) & 109.5 \\ C(29 A)-C(31 A)-H(31 D) & 109.5 \\ C(29 A)-C(31 A)-H(31 E) & 109.5 \\ H(31 D)-C(31 A)-H(31 E) & 109.5 \\ C(29 A)-C(31 A)-H(31 F) & 109.5 \\ H(31 D)-C(31 A)-H(31 F) & 109.5 \\ H(31 E)-C(31 A)-H(31 F) & 109.5 \\ C(34 A)-C(32 A)-C(26 A) & 112.6(5) \\ C(34 A)-C(32 A)-C(33 A) & 112.4(9) \\ C(26 A)-C(32 A)-C(33 A) & 109.8(8) \\ C(34 A)-C(32 A)-H(32 A) & 107.2 \\ C(26 A)-C(32 A)-H(32 A) & 107.2 \\ C(33 A)-C(32 A)-H(32 A) & 107.2 \\ C(32 A)-C(33 A)-H(33 D) & 109.5 \\ C(32 A)-C(33 A)-H(33 E) & 109.5 \\ H(33 D)-C(33 A)-H(33 E) & 109.5 \\ C(32 A)-C(33 A)-H(33 F) & 109.5 \\ H(33 D)-C(33 A)-H(33 F) & 109.5 \\ H(33 E)-C(33 A)-H(33 F) & 109.5 \\ C(32 A)-C(34 A)-H(34 D) & 109.5 \\ C(32 A)-C(34 A)-H(34 E) & 109.5 \\ H(34 D)-C(34 A)-H(34 E) & 109.5 \\ & \end{array}$

\begin{tabular}{|c|c|}
\hline$C(32 \mathrm{~A})-\mathrm{C}(34 \mathrm{~A})-\mathrm{H}(34 \mathrm{~F})$ & 109.5 \\
\hline$H(34 D)-C(34 A)-H(34 F)$ & 109.5 \\
\hline$H(34 E)-C(34 A)-H(34 F)$ & 109.5 \\
\hline$C(28 \mathrm{~A})-C(35 \mathrm{~A})-C(36 \mathrm{~A})$ & 111.1(5) \\
\hline$C(28 A)-C(35 A)-C(37 A)$ & $111.5(6)$ \\
\hline$C(36 A)-C(35 A)-C(37 A)$ & $109.7(6)$ \\
\hline$C(28 A)-C(35 A)-H(35 A)$ & 108.2 \\
\hline$C(36 A)-C(35 A)-H(35 A)$ & 108.2 \\
\hline$C(37 A)-C(35 A)-H(35 A)$ & 108.2 \\
\hline$C(35 A)-C(36 A)-H(36 D)$ & 109.5 \\
\hline$C(35 A)-C(36 A)-H(36 E)$ & 109.5 \\
\hline$H(36 D)-C(36 A)-H(36 E)$ & 109.5 \\
\hline$C(35 A)-C(36 A)-H(36 F)$ & 109.5 \\
\hline$H(36 D)-C(36 A)-H(36 F)$ & 109.5 \\
\hline$H(36 E)-C(36 A)-H(36 F)$ & 109.5 \\
\hline$C(35 A)-C(37 A)-H(37 D)$ & 109.5 \\
\hline$C(35 A)-C(37 A)-H(37 E)$ & 109.5 \\
\hline$H(37 D)-C(37 A)-H(37 E)$ & 109.5 \\
\hline$C(35 A)-C(37 A)-H(37 F)$ & 109.5 \\
\hline$H(37 D)-C(37 A)-H(37 F)$ & 109.5 \\
\hline$H(37 E)-C(37 A)-H(37 F)$ & 109.5 \\
\hline$C(14)-C(17)-H(17 A)$ & 109.5 \\
\hline$C(14)-C(17)-H(17 B)$ & 109.5 \\
\hline$H(17 A)-C(17)-H(17 B)$ & 109.5 \\
\hline $\mathrm{C}(14)-\mathrm{C}(17)-\mathrm{H}(17 \mathrm{C})$ & 109.5 \\
\hline$H(17 A)-C(17)-H(17 C)$ & 109.5 \\
\hline $\mathrm{H}(17 \mathrm{~B})-\mathrm{C}(17)-\mathrm{H}(17 \mathrm{C})$ & 109.5 \\
\hline $\mathrm{C}(41 \mathrm{~A})-\mathrm{N}(2)-\mathrm{Mo}(1)$ & $170.4(3)$ \\
\hline $\mathrm{C}(41)-\mathrm{N}(2)-\mathrm{Mo}(1)$ & $173.3(3)$ \\
\hline$N(2)-C(41)-C(42)$ & $116.0(7)$ \\
\hline$N(2)-C(41)-C(46)$ & $116.4(7)$ \\
\hline$C(42)-C(41)-C(46)$ & $127.5(7)$ \\
\hline$C(43)-C(42)-C(41)$ & $117.1(7)$ \\
\hline$C(43)-C(42)-C(47)$ & $115.3(6)$ \\
\hline$C(41)-C(42)-C(47)$ & $127.4(6)$ \\
\hline$C(44)-C(43)-C(42)$ & $119.6(6)$ \\
\hline$C(44)-C(43)-H(43)$ & 120.2 \\
\hline$C(42)-C(43)-H(43)$ & 120.2 \\
\hline$C(43)-C(44)-C(45)$ & $119.8(6)$ \\
\hline $\mathrm{C}(43)-\mathrm{C}(44)-\mathrm{H}(44)$ & 120.1 \\
\hline $\mathrm{C}(45)-\mathrm{C}(44)-\mathrm{H}(44)$ & 120.1 \\
\hline$C(44)-C(45)-C(46)$ & $129.0(7)$ \\
\hline $\mathrm{C}(44)-\mathrm{C}(45)-\mathrm{H}(45)$ & 115.5 \\
\hline$C(46)-C(45)-H(45)$ & 115.5 \\
\hline$C(45)-C(46)-C(41)$ & $105.8(7)$ \\
\hline$C(45)-C(46)-C(50)$ & $129.5(8)$ \\
\hline$C(41)-C(46)-C(50)$ & $124.7(7)$ \\
\hline$C(48)-C(47)-C(42)$ & $110.3(7)$ \\
\hline$C(48)-C(47)-C(49)$ & $111.0(7)$ \\
\hline$C(42)-C(47)-C(49)$ & $112.2(6)$ \\
\hline $\mathrm{C}(48)-\mathrm{C}(47)-\mathrm{H}(47)$ & 107.7 \\
\hline $\mathrm{C}(42)-\mathrm{C}(47)-\mathrm{H}(47)$ & 107.7 \\
\hline $\mathrm{C}(49)-\mathrm{C}(47)-\mathrm{H}(47)$ & 107.7 \\
\hline
\end{tabular}




\begin{tabular}{|c|c|}
\hline$C(47)-C(48)-H(48 A)$ & 109.5 \\
\hline $\mathrm{C}(47)-\mathrm{C}(48)-\mathrm{H}(48 \mathrm{~B})$ & 109.5 \\
\hline $\mathrm{H}(48 \mathrm{~A})-\mathrm{C}(48)-\mathrm{H}(48 \mathrm{~B})$ & 109.5 \\
\hline $\mathrm{C}(47)-\mathrm{C}(48)-\mathrm{H}(48 \mathrm{C})$ & 109.5 \\
\hline$H(48 A)-C(48)-H(48 C)$ & 109.5 \\
\hline $\mathrm{H}(48 \mathrm{~B})-\mathrm{C}(48)-\mathrm{H}(48 \mathrm{C})$ & 109.5 \\
\hline $\mathrm{C}(47)-\mathrm{C}(49)-\mathrm{H}(49 \mathrm{~A})$ & 109.5 \\
\hline $\mathrm{C}(47)-\mathrm{C}(49)-\mathrm{H}(49 \mathrm{~B})$ & 109.5 \\
\hline$H(49 A)-C(49)-H(49 B)$ & 109.5 \\
\hline$C(47)-C(49)-H(49 C)$ & 109.5 \\
\hline$H(49 A)-C(49)-H(49 C)$ & 109.5 \\
\hline$H(49 B)-C(49)-H(49 C)$ & 109.5 \\
\hline$C(46)-C(50)-C(52)$ & $120.5(8)$ \\
\hline$C(46)-C(50)-C(51)$ & $114.3(8)$ \\
\hline$C(52)-C(50)-C(51)$ & $105.8(6)$ \\
\hline$C(46)-C(50)-H(50)$ & 104.9 \\
\hline$C(52)-C(50)-H(50)$ & 104.9 \\
\hline$C(51)-C(50)-H(50)$ & 104.9 \\
\hline$C(50)-C(51)-H(51 A)$ & 109.5 \\
\hline$C(50)-C(51)-H(51 B)$ & 109.5 \\
\hline$H(51 A)-C(51)-H(51 B)$ & 109.5 \\
\hline$C(50)-C(51)-H(51 C)$ & 109.5 \\
\hline$H(51 A)-C(51)-H(51 C)$ & 109.5 \\
\hline$H(51 B)-C(51)-H(51 C)$ & 109.5 \\
\hline$C(50)-C(52)-H(52 A)$ & 109.5 \\
\hline$C(50)-C(52)-H(52 B)$ & 109.5 \\
\hline$H(52 A)-C(52)-H(52 B)$ & 109.5 \\
\hline$C(50)-C(52)-H(52 C)$ & 109.5 \\
\hline$H(52 A)-C(52)-H(52 C)$ & 109.5 \\
\hline $\mathrm{H}(52 \mathrm{~B})-\mathrm{C}(52)-\mathrm{H}(52 \mathrm{C})$ & 109.5 \\
\hline$C(46 A)-C(41 A)-C(42 A)$ & $112.4(8)$ \\
\hline$C(46 A)-C(41 A)-N(2)$ & $123.8(8)$ \\
\hline$C(42 A)-C(41 A)-N(2)$ & $123.8(8)$ \\
\hline$C(41 A)-C(42 A)-C(43 A)$ & $119.2(7)$ \\
\hline$C(41 A)-C(42 A)-C(47 A)$ & $118.0(7)$ \\
\hline$C(43 A)-C(42 A)-C(47 A)$ & $122.8(7)$ \\
\hline$C(44 A)-C(43 A)-C(42 A)$ & $124.1(7)$ \\
\hline$C(44 A)-C(43 A)-H(43 A)$ & 117.9 \\
\hline$C(42 A)-C(43 A)-H(43 A)$ & 117.9 \\
\hline$C(45 A)-C(44 A)-C(43 A)$ & $119.6(7)$ \\
\hline$C(45 A)-C(44 A)-H(44 A)$ & 120.2 \\
\hline$C(43 A)-C(44 A)-H(44 A)$ & 120.2 \\
\hline$C(44 A)-C(45 A)-C(46 A)$ & $112.0(8)$ \\
\hline$C(44 A)-C(45 A)-H(45 A)$ & 124.0 \\
\hline$C(46 A)-C(45 A)-H(45 A)$ & 124.0 \\
\hline$C(41 A)-C(46 A)-C(45 A)$ & 131.6(9) \\
\hline$C(41 A)-C(46 A)-C(50 A)$ & 119.1(9) \\
\hline$C(45 A)-C(46 A)-C(50 A)$ & 108.9(9) \\
\hline$C(42 A)-C(47 A)-C(48 A)$ & $110.0(7)$ \\
\hline$C(42 A)-C(47 A)-C(49 A)$ & $111.3(6)$ \\
\hline$C(48 A)-C(47 A)-C(49 A)$ & 111.1(6) \\
\hline$C(42 A)-C(47 A)-H(47 A)$ & 108.1 \\
\hline$C(48 A)-C(47 A)-H(47 A)$ & 108.1 \\
\hline
\end{tabular}

\begin{tabular}{|c|c|}
\hline$C(49 A)-C(47 A)-H(47 A)$ & 108.1 \\
\hline$C(47 A)-C(48 A)-H(48 D)$ & 109.5 \\
\hline$C(47 A)-C(48 A)-H(48 E)$ & 109.5 \\
\hline$H(48 D)-C(48 A)-H(48 E)$ & 109.5 \\
\hline$C(47 A)-C(48 A)-H(48 F)$ & 109.5 \\
\hline $\mathrm{H}(48 \mathrm{D})-\mathrm{C}(48 \mathrm{~A})-\mathrm{H}(48 \mathrm{~F})$ & 109.5 \\
\hline$H(48 E)-C(48 A)-H(48 F)$ & 109.5 \\
\hline$C(47 A)-C(49 A)-H(49 D)$ & 109.5 \\
\hline$C(47 A)-C(49 A)-H(49 E)$ & 109.5 \\
\hline$H(49 D)-C(49 A)-H(49 E)$ & 109.5 \\
\hline$C(47 A)-C(49 A)-H(49 F)$ & 109.5 \\
\hline$H(49 D)-C(49 A)-H(49 F)$ & 109.5 \\
\hline$H(49 E)-C(49 A)-H(49 F)$ & 109.5 \\
\hline$C(46 A)-C(50 A)-C(52 A)$ & $104.3(10)$ \\
\hline$C(46 A)-C(50 A)-C(51 A)$ & $103.5(9)$ \\
\hline$C(52 A)-C(50 A)-C(51 A)$ & $114.4(9)$ \\
\hline$C(46 A)-C(50 A)-H(50 A)$ & 111.4 \\
\hline$C(52 A)-C(50 A)-H(50 A)$ & 111.4 \\
\hline$C(51 A)-C(50 A)-H(50 A)$ & 111.4 \\
\hline$C(50 A)-C(51 A)-H(51 D)$ & 109.5 \\
\hline$C(50 A)-C(51 A)-H(51 E)$ & 109.5 \\
\hline$H(51 D)-C(51 A)-H(51 E)$ & 109.5 \\
\hline$C(50 A)-C(51 A)-H(51 F)$ & 109.5 \\
\hline$H(51 D)-C(51 A)-H(51 F)$ & 109.5 \\
\hline$H(51 E)-C(51 A)-H(51 F)$ & 109.5 \\
\hline$C(50 A)-C(52 A)-H(52 D)$ & 109.5 \\
\hline$C(50 A)-C(52 A)-H(52 E)$ & 109.5 \\
\hline$H(52 D)-C(52 A)-H(52 E)$ & 109.5 \\
\hline$C(50 A)-C(52 A)-H(52 F)$ & 109.5 \\
\hline$H(52 D)-C(52 A)-H(52 F)$ & 109.5 \\
\hline$H(52 E)-C(52 A)-H(52 F)$ & 109.5 \\
\hline$C(61)-C(62)-H(62 A)$ & 109.5 \\
\hline$C(61)-C(62)-H(62 B)$ & 109.5 \\
\hline$H(62 A)-C(62)-H(62 B)$ & 109.5 \\
\hline$C(61)-C(62)-H(62 C)$ & 109.5 \\
\hline$H(62 A)-C(62)-H(62 C)$ & 109.5 \\
\hline $\mathrm{H}(62 \mathrm{~B})-\mathrm{C}(62)-\mathrm{H}(62 \mathrm{C})$ & 109.5 \\
\hline$O(5)-C(61)-C(62)$ & $112.1(3)$ \\
\hline$O(5)-C(61)-H(61 A)$ & 109.2 \\
\hline$C(62)-C(61)-H(61 A)$ & 109.2 \\
\hline $\mathrm{O}(5)-\mathrm{C}(61)-\mathrm{H}(61 \mathrm{~B})$ & 109.2 \\
\hline$C(62)-C(61)-H(61 B)$ & 109.2 \\
\hline$H(61 A)-C(61)-H(61 B)$ & 107.9 \\
\hline$C(63)-O(5)-C(61)$ & $114.1(7)$ \\
\hline$C(61)-O(5)-C(63 A)$ & $114.5(7)$ \\
\hline $\mathrm{C}(63)-\mathrm{O}(5)-\mathrm{Mo}(1)$ & $125.9(6)$ \\
\hline $\mathrm{C}(61)-\mathrm{O}(5)-\mathrm{Mo}(1)$ & $120.01(16)$ \\
\hline $\mathrm{C}(63 \mathrm{~A})-\mathrm{O}(5)-\mathrm{Mo}(1)$ & $123.6(7)$ \\
\hline$O(5)-C(63)-C(64)$ & $109.6(7)$ \\
\hline$O(5)-C(63)-H(63 A)$ & 109.8 \\
\hline$C(64)-C(63)-H(63 A)$ & 109.8 \\
\hline $\mathrm{O}(5)-\mathrm{C}(63)-\mathrm{H}(63 \mathrm{~B})$ & 109.8 \\
\hline $\mathrm{C}(64)-\mathrm{C}(63)-\mathrm{H}(63 \mathrm{~B})$ & 109.8 \\
\hline
\end{tabular}




$\begin{array}{ll}H(63 A)-C(63)-H(63 B) & 108.2 \\ C(63)-C(64)-H(64 A) & 109.5 \\ C(63)-C(64)-H(64 B) & 109.5 \\ H(64 A)-C(64)-H(64 B) & 109.5 \\ C(63)-C(64)-H(64 C) & 109.5 \\ H(64 A)-C(64)-H(64 C) & 109.5 \\ H(64 B)-C(64)-H(64 C) & 109.5 \\ O(5)-C(63 A)-C(64 A) & 114.5(10) \\ \text { O(5)-C(63A)-H(63C) } & 108.6 \\ C(64 A)-C(63 A)-H(63 C) & 108.6 \\ O(5)-C(63 A)-H(63 D) & 108.6 \\ C(64 A)-C(63 A)-H(63 D) & 108.6 \\ H(63 C)-C(63 A)-H(63 D) & 107.6 \\ C(63 A)-C(64 A)-H(64 D) & 109.5 \\ C(63 A)-C(64 A)-H(64 E) & 109.5 \\ H(64 D)-C(64 A)-H(64 E) & 109.5 \\ C(63 A)-C(64 A)-H(64 F) & 109.5 \\ H(64 D)-C(64 A)-H(64 F) & 109.5 \\ H(64 E)-C(64 A)-H(64 F) & 109.5 \\ C(1 S)-C(2 S)-H(2 S 1) & 109.5 \\ C(1 S)-C(2 S)-H(2 S 2) & 109.5 \\ H(2 S 1)-C(2 S)-H(2 S 2) & 109.5 \\ C(1 S)-C(2 S)-H(2 S 3) & 109.5 \\ H(2 S 1)-C(2 S)-H(2 S 3) & 109.5 \\ H(2 S 2)-C(2 S)-H(2 S 3) & 109.5 \\ C(2 S)-C(1 S)-O(1 S) & 108.3(8) \\ C(2 S)-C(1 S)-H(1 S 1) & 110.0 \\ O(1 S)-C(1 S)-H(1 S 1) & 110.0 \\ C(2 S)-C(1 S)-H(1 S 2) & 110.0 \\ O(1 S)-C(1 S)-H(1 S 2) & 110.0 \\ H(1 S 1)-C(1 S)-H(1 S 2) & 108.4 \\ C(3 S)-O(1 S)-C(1 S) & 116.6(7) \\ C(4 S)-C(3 S)-O(1 S) & 111.3(8) \\ C(4 S)-C(3 S)-H(3 S 1) & 109.4 \\ O(1 S)-C(3 S)-H(3 S 1) & 109.4 \\ C(4 S)-C(3 S)-H(3 S 2) & 109.4 \\ O(1 S)-C(3 S)-H(3 S 2) & 109.4 \\ H(3 S 1)-C(3 S)-H(3 S 2) & 108.0 \\ C(3 S)-C(4 S)-H(4 S 1) & 109.5 \\ C(3 S)-C(4 S)-H(4 S 2) & 109.5 \\ H(4 S 1)-C(4 S)-H(4 S 2) & 109.5 \\ C(3 S)-C(4 S)-H(4 S 3) & 109.5 \\ H(4 S 1)-C(4 S)-H(4 S 3) & 109.5 \\ H(4 S 2)-C(4 S)-H(4 S 3) & 109.5 \\ C(1 T)-C(2 T)-H(2 T 1) & 109.5 \\ C(1 T)-C(2 T)-H(2 T 2) & 109.5 \\ H(2 T 1)-C(2 T)-H(2 T 2) & 109.5 \\ C(1 T)-C(2 T)-H(2 T 3) & 109.5 \\ H(2 T 1)-C(2 T)-H(2 T 3) & 109.5 \\ H(2 T 2)-C(2 T)-H(2 T 3) & 109.5 \\ C(2 T)-C(1 T)-O(1 T) & 106.9(8) \\ C(2 T)-C(1 T)-H(1 T 1) & 110.3 \\ O(11 T)-C(1 T)-H(1 T 1) & 110.3 \\ & \\ & \end{array}$

\begin{tabular}{|c|c|}
\hline$C(2 T)-C(1 T)-H(1 T 2)$ & 110.3 \\
\hline $\mathrm{O}(1 \mathrm{~T})-\mathrm{C}(1 \mathrm{~T})-\mathrm{H}(1 \mathrm{~T} 2)$ & 110.3 \\
\hline $\mathrm{H}(1 \mathrm{~T} 1)-\mathrm{C}(1 \mathrm{~T})-\mathrm{H}(1 \mathrm{~T} 2)$ & 108.6 \\
\hline$C(1 T)-O(1 T)-C(3 T)$ & $116.9(7)$ \\
\hline$C(4 T)-C(3 T)-O(1 T)$ & 109.7(9) \\
\hline $\mathrm{C}(4 \mathrm{~T})-\mathrm{C}(3 \mathrm{~T})-\mathrm{H}(3 \mathrm{~T} 1)$ & 109.7 \\
\hline $\mathrm{O}(1 \mathrm{~T})-\mathrm{C}(3 \mathrm{~T})-\mathrm{H}(3 \mathrm{~T} 1)$ & 109.7 \\
\hline $\mathrm{C}(4 \mathrm{~T})-\mathrm{C}(3 \mathrm{~T})-\mathrm{H}(3 \mathrm{~T} 2)$ & 109.7 \\
\hline $\mathrm{O}(1 \mathrm{~T})-\mathrm{C}(3 \mathrm{~T})-\mathrm{H}(3 \mathrm{~T} 2)$ & 109.7 \\
\hline $\mathrm{H}(3 \mathrm{~T} 1)-\mathrm{C}(3 \mathrm{~T})-\mathrm{H}(3 \mathrm{~T} 2)$ & 108.2 \\
\hline $\mathrm{C}(3 \mathrm{~T})-\mathrm{C}(4 \mathrm{~T})-\mathrm{H}(4 \mathrm{~T} 1)$ & 109.5 \\
\hline$C(3 T)-C(4 T)-H(4 T 2)$ & 109.5 \\
\hline $\mathrm{H}(4 \mathrm{~T} 1)-\mathrm{C}(4 \mathrm{~T})-\mathrm{H}(4 \mathrm{~T} 2)$ & 109.5 \\
\hline$C(3 T)-C(4 T)-H(4 T 3)$ & 109.5 \\
\hline $\mathrm{H}(4 \mathrm{~T} 1)-\mathrm{C}(4 \mathrm{~T})-\mathrm{H}(4 \mathrm{~T} 3)$ & 109.5 \\
\hline $\mathrm{H}(4 \mathrm{~T} 2)-\mathrm{C}(4 \mathrm{~T})-\mathrm{H}(4 \mathrm{~T} 3)$ & 109.5 \\
\hline$C(1 U)-C(2 U)-H(2 U 1)$ & 109.5 \\
\hline$C(1 U)-C(2 U)-H(2 U 2)$ & 109.5 \\
\hline $\mathrm{H}(2 \mathrm{U} 1)-\mathrm{C}(2 \mathrm{U})-\mathrm{H}(2 \mathrm{U} 2)$ & 109.5 \\
\hline$C(1 U)-C(2 U)-H(2 U 3)$ & 109.5 \\
\hline $\mathrm{H}(2 \mathrm{U} 1)-\mathrm{C}(2 \mathrm{U})-\mathrm{H}(2 \mathrm{U} 3)$ & 109.5 \\
\hline $\mathrm{H}(2 \mathrm{U} 2)-\mathrm{C}(2 \mathrm{U})-\mathrm{H}(2 \mathrm{U} 3)$ & 109.5 \\
\hline$C(2 U)-C(1 U)-O(1 U)$ & 111.2(9) \\
\hline$C(2 U)-C(1 U)-H(1 U 1)$ & 109.4 \\
\hline $\mathrm{O}(1 \mathrm{U})-\mathrm{C}(1 \mathrm{U})-\mathrm{H}(1 \mathrm{U} 1)$ & 109.4 \\
\hline$C(2 U)-C(1 U)-H(1 U 2)$ & 109.4 \\
\hline $\mathrm{O}(1 \mathrm{U})-\mathrm{C}(1 \mathrm{U})-\mathrm{H}(1 \cup 2)$ & 109.4 \\
\hline $\mathrm{H}(1 \cup 1)-\mathrm{C}(1 \mathrm{U})-\mathrm{H}(1 \cup 2)$ & 108.0 \\
\hline$C(1 U)-O(1 U)-C(3 U)$ & 117.1(12) \\
\hline$C(4 U)-C(3 U)-O(1 U)$ & $105.9(11)$ \\
\hline$C(4 U)-C(3 U)-H(3 U 1)$ & 110.6 \\
\hline $\mathrm{O}(1 \mathrm{U})-\mathrm{C}(3 \mathrm{U})-\mathrm{H}(3 \mathrm{U} 1)$ & 110.6 \\
\hline $\mathrm{C}(4 U)-\mathrm{C}(3 \mathrm{U})-\mathrm{H}(3 \mathrm{U} 2)$ & 110.6 \\
\hline $\mathrm{O}(1 \mathrm{U})-\mathrm{C}(3 \mathrm{U})-\mathrm{H}(3 \mathrm{U} 2)$ & 110.6 \\
\hline $\mathrm{H}(3 \mathrm{U} 1)-\mathrm{C}(3 \mathrm{U})-\mathrm{H}(3 \mathrm{U} 2)$ & 108.7 \\
\hline$C(3 U)-C(4 U)-H(4 U 1)$ & 109.5 \\
\hline$C(3 U)-C(4 U)-H(4 U 2)$ & 109.5 \\
\hline $\mathrm{H}(4 \mathrm{U} 1)-\mathrm{C}(4 \mathrm{U})-\mathrm{H}(4 \mathrm{U} 2)$ & 109.5 \\
\hline$C(3 U)-C(4 U)-H(4 U 3)$ & 109.5 \\
\hline $\mathrm{H}(4 \mathrm{U} 1)-\mathrm{C}(4 \mathrm{U})-\mathrm{H}(4 \mathrm{U} 3)$ & 109.5 \\
\hline $\mathrm{H}(4 \mathrm{U} 2)-\mathrm{C}(4 \mathrm{U})-\mathrm{H}(4 \mathrm{U} 3)$ & 109.5 \\
\hline
\end{tabular}




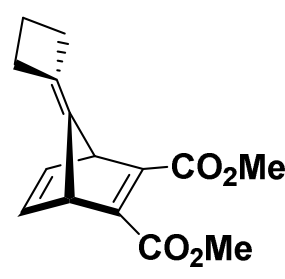

A

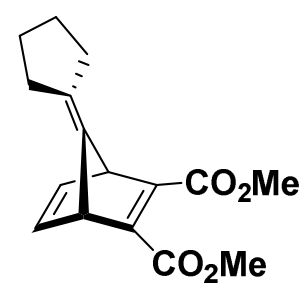

B

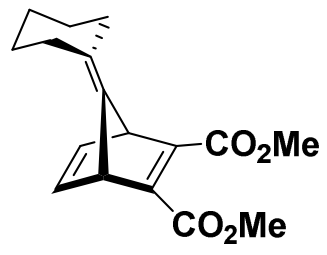

C

Figure S13. Monomers explored in this study were synthesized according to literature procedures. ${ }^{11}$

Table S11. ROMP of A, B, and C. ${ }^{a}$

\begin{tabular}{c|c|c|c|c|c|c|c|c}
\hline Cat. $^{\boldsymbol{a}}$ & Solvent & Temp. $\left(^{\circ} \mathbf{C}\right)$ & Time (h) & Monomer & Conv.(\%) & Mn & Mw & PDI \\
\hline $\mathbf{1}($ MesON) & Tol- $d_{8}$ & 100 & 1 & A & $100(>99)$ & $1.3 * 10^{5}$ & $3.4 * 10^{5}$ & 2.5 \\
$\mathbf{1}($ MesON) & Tol- $d_{8}$ & 100 & 1 & B & $100(>99)$ & $1.8 * 10^{5}$ & $3.5 * 10^{5}$ & 2.0 \\
$\mathbf{1 ( M e s O N )}$ & ${\text { Tol- } d_{8}}$ & 100 & 1 & C & $100(>99)$ & $8.4 * 10^{4}$ & $1.7 * 10^{5}$ & 2.0
\end{tabular}

${ }^{a}$ The polymers were subjected to GPC analysis at $60{ }^{\circ} \mathrm{C}$ in $0.025 \mathrm{M} \mathrm{LiBr}$ DMF solution. The refractive index, $\mathrm{dn} / \mathrm{dc}(\mathrm{mL} / \mathrm{g}) \mathrm{of}$ 0.1850 was used in the measurements.

${ }^{b}$ Isolated yields in parentheses.

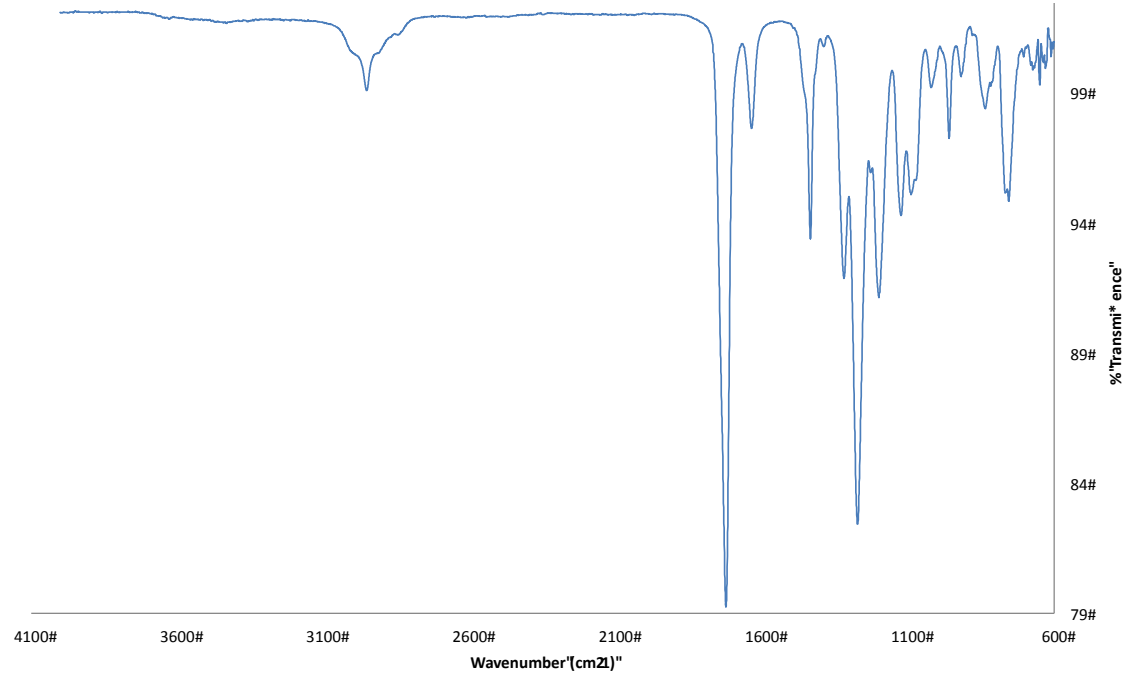

Figure S14. IR spectrum for cis,tactic poly(A). 


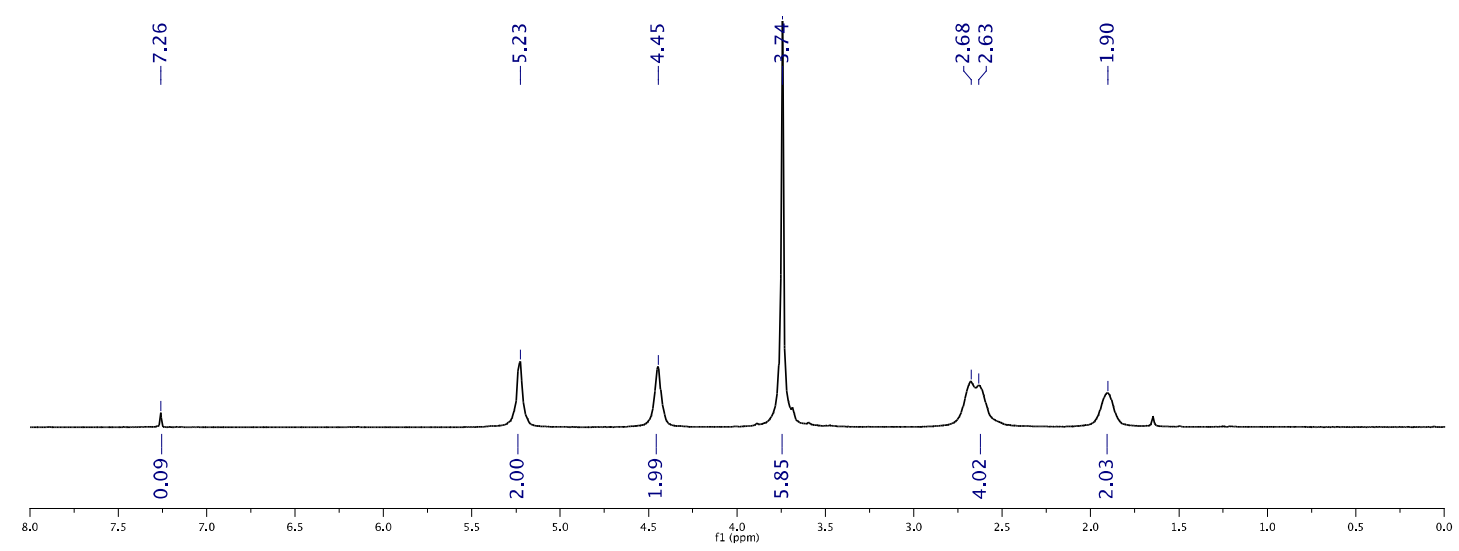

Figure S15. ${ }^{1} \mathrm{H}$ NMR $\left(500 \mathrm{MHz}, \mathrm{CDCl}_{3}\right)$ spectrum of cis, tactic poly(A).

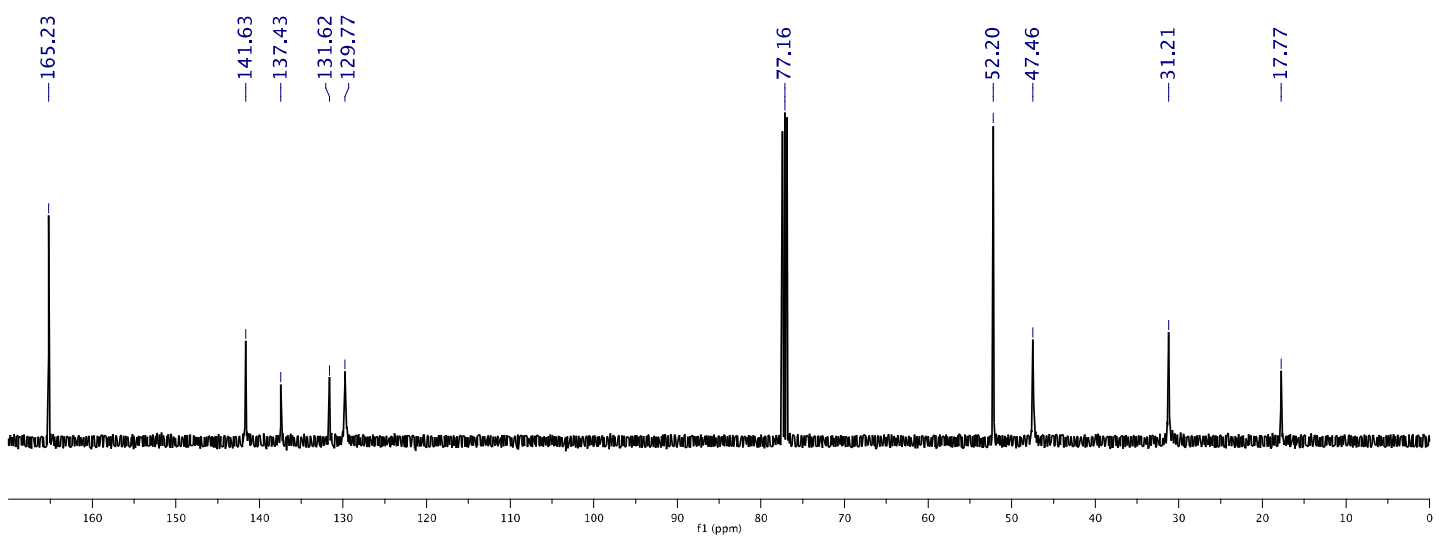

Figure S16. ${ }^{13} \mathrm{C}\left\{{ }^{1} \mathrm{H}\right\} \mathrm{NMR}\left(\mathrm{CDCl}_{3}, 125 \mathrm{MHz}\right)$ spectrum of cis,tactic poly(A).

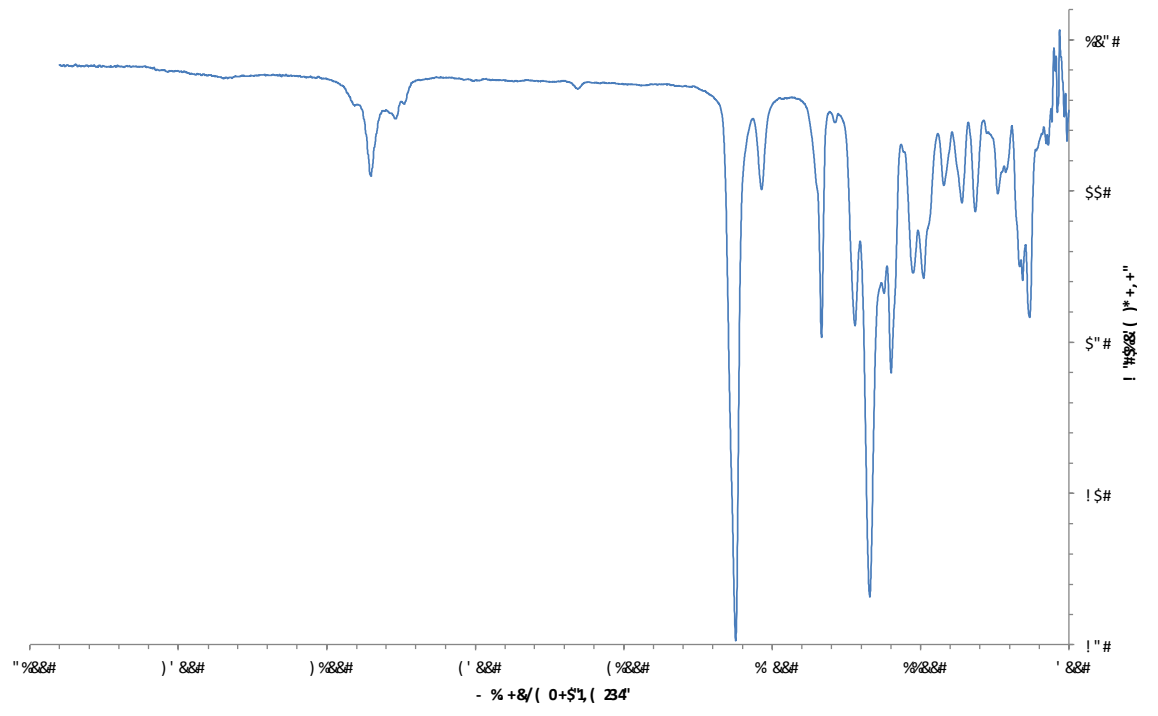

Figure S17. IR spectrum for cis,tactic poly(B). 


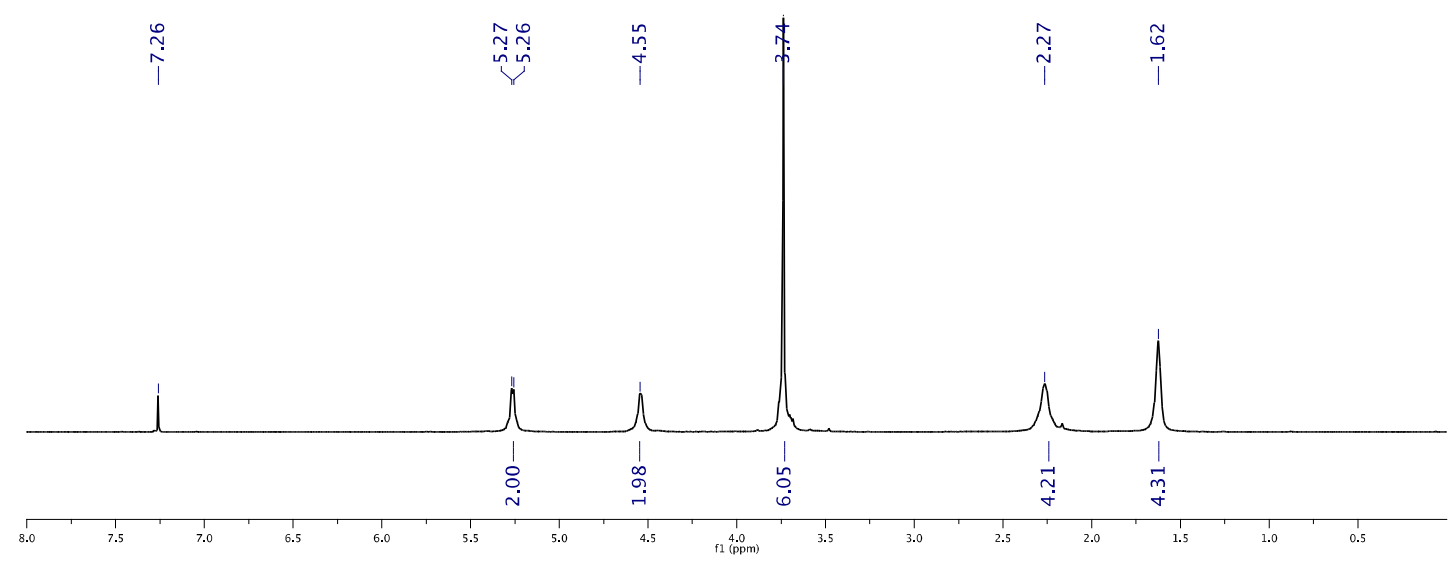

Figure S18. ${ }^{1} \mathrm{H}$ NMR $\left(500 \mathrm{MHz}, \mathrm{CDCl}_{3}\right)$ of cis, tactic poly(B).

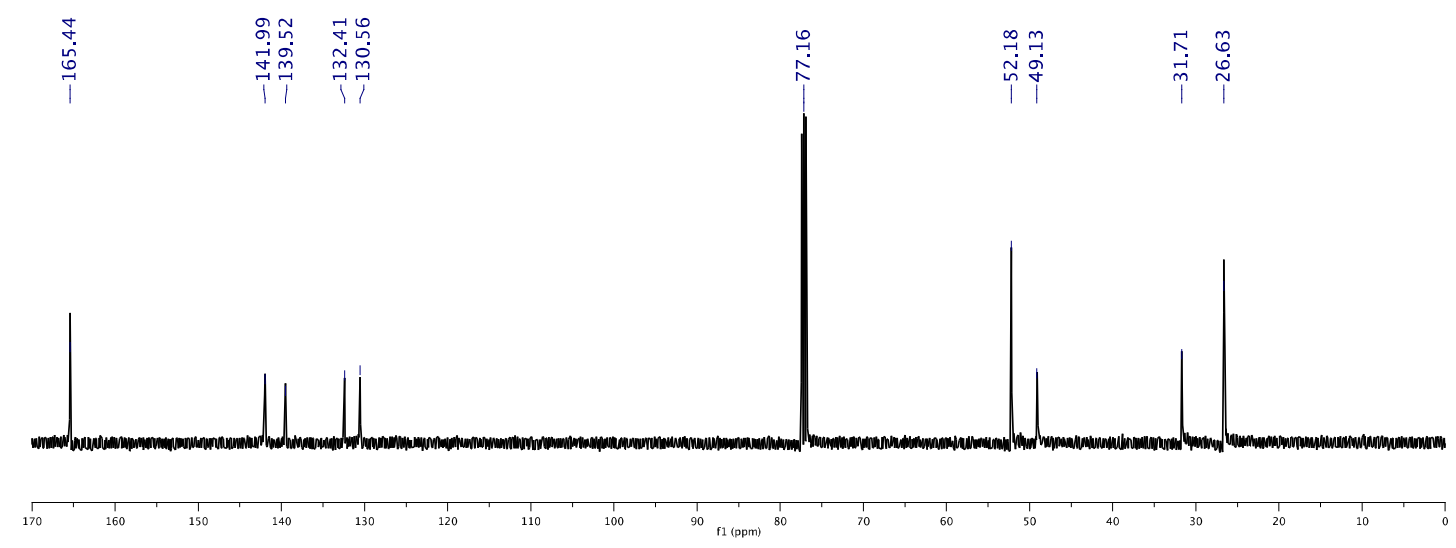

Figure S19. ${ }^{13} \mathrm{C} \mathrm{NMR}\left(\mathrm{CDCl}_{3}, 125 \mathrm{MHz}\right)$ of cis,tactic poly(B).

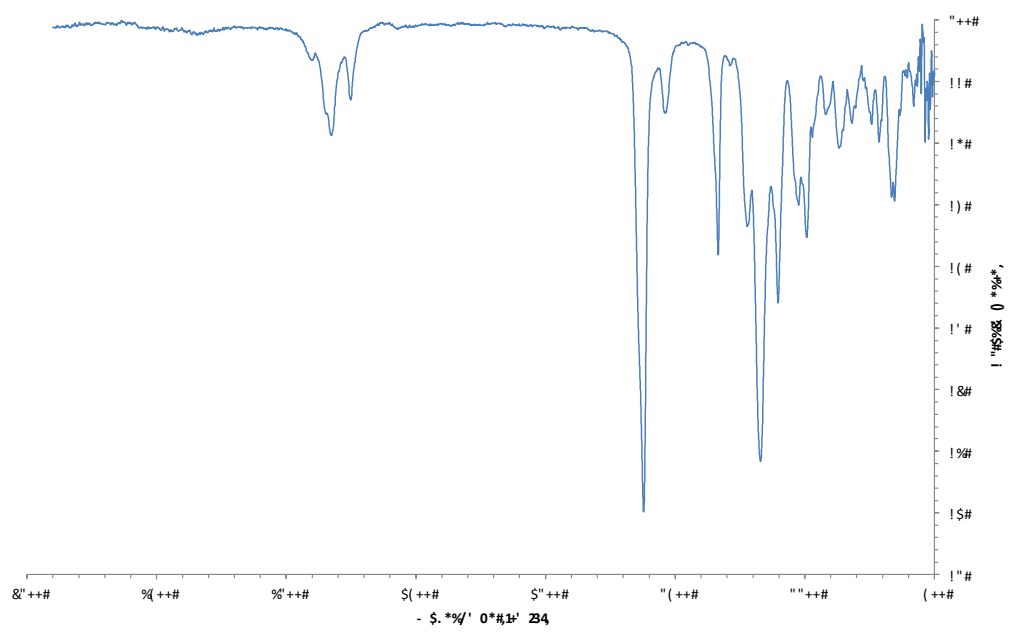

Figure S20. IR spectrum for cis,tactic poly(C). 


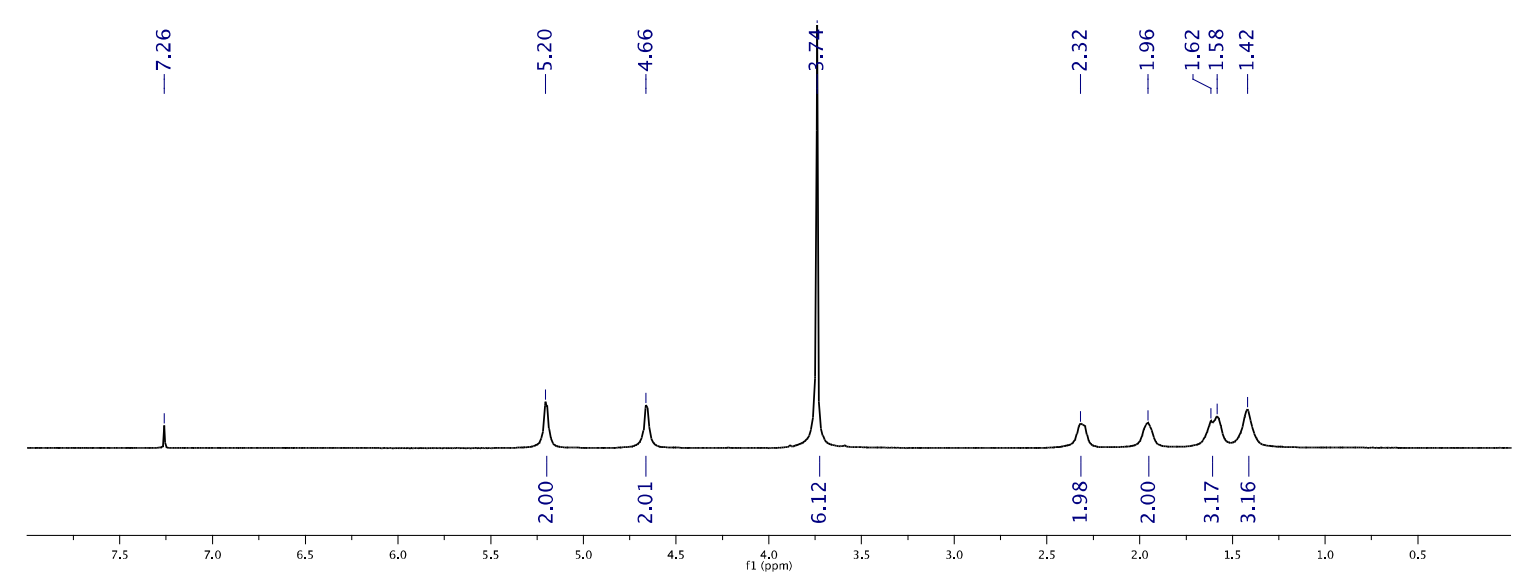

Figure S21. ${ }^{1} \mathrm{H} \mathrm{NMR}\left(\mathrm{CDCl}_{3}, 500 \mathrm{MHz}\right)$ of cis, tactic poly(C).

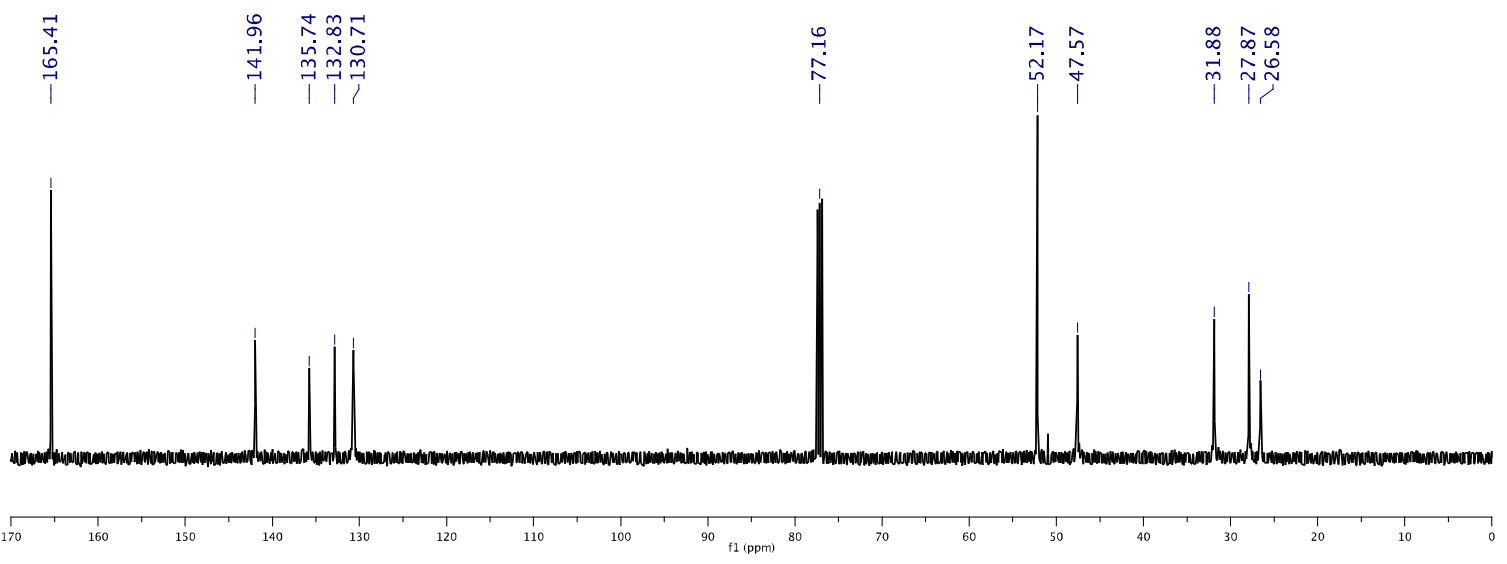

Figure S22. ${ }^{13} \mathrm{C} \mathrm{NMR}\left(\mathrm{CDCl}_{3}, 125 \mathrm{MHz}\right)$ of cis,tactic poly(C).

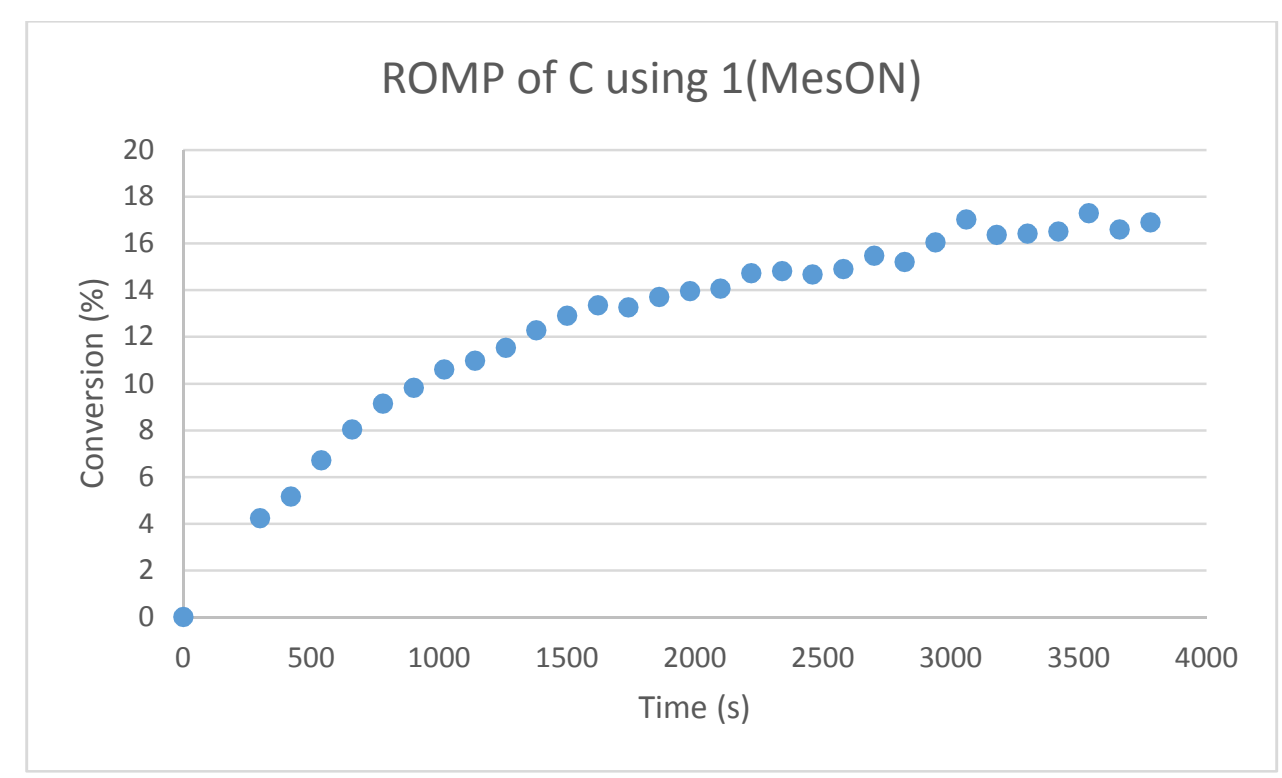

Figure S23. Representative reaction profile of the ROMP of monomer C (75 eq.) using $\mathbf{1}$ (MesON) at 100 ${ }^{\circ} \mathrm{C}$. The rate of monomer consumption did not correspond to zero, first, or second order. 


\section{References}

(1) Garcia, C. A. M.; Koch, E. K.; Löfstedt, A. J.; Cheng, A.; Gordon, S.; Hansson, T. F. Novel pharmaceutical compositions. PCT Int. Appl., 2007128492 A1, November 15, 2007.

(2) Wang, X.; Snieckus, V. Tetrahedron Lett. 1991, 32, 4879-4882.

(3) Koch, F.; Schubert, H.; Sirsch, P.; Berkefeld, A. Dalton Trans. 2015, 44, 13315-13324.

(4) (a) Omura, K.; J. Org. Chem. 1984, 49, 3046-3050. (b) Kometani, T.; Watt, D. S. J. Org. Chem. 1985, 50, 5384-5387.

(5) Sheldrick, G. M. Acta Cryst. 2015, A71, 3-8.

(6) Sheldrick, G. M. Acta Cryst. 2015, C71, 3-8.

(7) Müller, P. Crystallography Reviews 2009, 15, 57-83.

(8) Sheldrick, G. M (2008). CELL_NOW, University of Göttingen, Germany.

(9) Bruker (2010). SAINT, Bruker-AXS Inc., Madison, Wisconsin, USA.

(10) Sheldrick, G. M. (2008). TWINABS, University of Göttingen, Germany.

(11) Jeong, H.; John, J. M.; Schrock, R. R. Organometallics 2016, 34, 5136-5145. 